\title{
Forecasting Realized Volatility of Agricultural Commodities ${ }^{\text {th }}$
}

\author{
Stavros Degiannakis ${ }^{\mathrm{a}, \mathrm{b}}$, George Filis ${ }^{\mathrm{b}, *}$, Tony Klein ${ }^{\mathrm{c}}$, Thomas Walther ${ }^{\mathrm{d}, \mathrm{e}}$ \\ ${ }^{a}$ Department of Economics and Regional Development, Panteion University of Social and Political \\ Sciences, Greece \\ ${ }^{b}$ Department of Accounting, Finance and Economics, Bournemouth University, UK \\ 'Queen's Management School, Queen's University Belfast, UK \\ ${ }^{d}$ Utrecht School of Economics, Utrecht University, The Netherlands \\ ${ }^{e}$ Faculty of Business and Economics, Technische Universität Dresden, Germany
}

\begin{abstract}
We forecast the realized and median realized volatility of agricultural commodities using variants of the Heterogeneous AutoRegressive (HAR) model. We obtain tick-by-tick data for five widely traded agricultural commodities (Corn, Rough Rice, Soybeans, Sugar, and Wheat) from the CME/ICE. Real out-of-sample forecasts are produced for 1- up to 66-days ahead. Our in-sample analysis shows that the variants of the HAR model which decompose volatility measures into their continuous path and jump components and incorporate leverage effects offer better fitting in the predictive regressions. However, we convincingly demonstrate that such HAR extensions do not offer any superior predictive ability in the out-of-sample results, since none of these extensions produce significantly better forecasts compared to the simple HAR model. Our results remain robust even when we evaluate them in a Value-at-Risk framework. Thus, there is no benefit by adding more complexity, related to volatility decomposition or relative transformations of volatility, in the forecasting models.
\end{abstract}

Keywords: Agricultural Commodities, Realized Volatility, Median Realized Volatility, Heterogeneous Autoregressive model, Forecast.

JEL classification: C22; C53; Q02; Q17

\section{Introduction \& Brief Review of the Literature}

Examining the behaviour of agricultural commodity prices and volatilities is of significant importance since they represent a major component of household consumption. They also have a pronounced impact on food security, which primarily affects the poorer parts of the population (Ordu et aL, 2018).

\footnotetext{
The authors would like to thank the Guest Editor Tao Hong and three anonymous reviewers for their helpful comments on a previous version of this paper. We are thankful for the comments and support of Matthias Fengler and Karl Frauendorfer. George Filis and Stavros Degiannakis acknowledge the support of Bournemouth University, which provided funding for the purchase of the data under the University's QR funds. Part of the work has been conducted during Thomas Walther's research time as Assistant Professor at the University of St. Gallen, Institute for Operations Research and Computational Finance.

*Corresponding Author, Mail: gfilis@bournemouth.ac.uk
} 
The Food and Agricultural Organisation (FAO) of the United Nations (2010) have claimed that food prices had rarely experienced any significant volatility prior to 2008 (FAO), 2010); however, over the last decade (2008-2018) agricultural commodities have experienced enormous price swings resulting in both high and low volatility regimes (Greb \&. Prakash, 2015). This new normal suggests that the food system is becoming progressively more vulnerable to price volatility (FAO), 2010) and this led the G20 to request a report from several international bodies (including the World Bank, IMF, UNCTAD, OECD, and FAO, among others) in order "to develop options for G20 consideration on how to better mitigate and manage the risks associated with the price volatility of food and other agriculture commodities, without distorting market behaviour, ultimately to protect the most vulnerable." (FAC), 2010, p.3).

The Council on Foreign Relations (CFR, 2017) promotes that such increased volatility is the result of extreme weather events, biofuels production, market speculation but also rising demand coupled with declines in food stocks. von Braun \& Tadesse (2012) also show that agricultural commodities price volatility is impacted by the increasing linkages among agricultural prices, energy commodities, and financial markets. Ordu et al (2018) further suggest that the agricultural market is becoming financialized since institutional investors are increasing their holdings in the commodity markets, which further suggests the rise in the speculative activity in this market. It is rather easy to understand that such cross-market linkages and financialization processes could have destabilizing effects on agricultural food prices.

Proper modeling and detection of long-memory dynamics in the volatility of commodity futures improves risk-management techniques, such as volatility forecasting and hedging performance, and better characterizes equilibrium relationships. Over the last few years there is an increased effort either to model agricultural price volatility (focusing primarily on GARCH-type and wavelet-based modelling approaches) or to provide evidence of potential predictors of such volatility, within an in-sample setting (Egelkraut \& Garcia, 2006, EIder \& . lin, 20107, Anderluh \& Borovkova, 20108, Triantafyllou et al., 2015, Li et al., 2(17).

Given the aforementioned market conditions and previous research effort to model agricultural volatility, it becomes central to develop the necessary frameworks that would allow successful forecasts for agricultural commodity price volatility so that policy institutions can get prepared for high price volatility periods or design preventing policies, as also implied by Greb \& Prakash (2017).

Despite the recent evidence provided by policy institutions for the need of successful agricultural price volatility forecasts, the fact that modelling approaches for agricultural price volatility have developed for over 15 years now, as well as, the fact that the first effort to produce real out-of-sample forecasts was by Giot \& Laurent (2003), we observe the paradox that there are only four other recent studies in this strand of the literature, those by Tian et al. (2017a,b), Yang et al. (2017), and Lun et al. (2019). 
Starting with the former study, Giot \& Laurent (2003) focus on Cocoa, Coffee, and Sugar futures price volatility and use GARCH-type models to generate the forecasts. By contrast, Tian et al. (2017a), Tian et al. (2017b) and Yang et al. (2017) utilize the increased availability of ultra-high frequency data and extend Corsi (20)(1) Heterogeneous AutoRegressive (HAR) model to produce short-run volatility forecasts (up to 20-days ahead).

More specifically, Tian et a.. (2017a) use a two regime-switching Markov models to forecast realized volatility for five agricultural commodities traded in the Chinese market, namely, Soybean, Soybean oil, White Sugar, Gluten Wheat and Cotton. They find evidence that regime switching dynamics offer predictive gains compared to both a simple AR(1) and a Markov-Switching AR(1) model. Yang et al. (2017) also use intra-day data from the Chinese commodity futures markets (Zhenzhou Commodity Exchange and Dalian Commodity Exchange) of Soybean, Cotton, Gluten Wheat and Corn futures prices and employ a similar strategy with Tian et al. (2017b), where the HAR model is extended with potential predictors (such as day-of-the-week dummies, past cumulative returns and the jump component) and forecasts are generated based on bagging and combination methods. Their conclusions suggest the forecasts based on the HAR models with bagging and principal component combination methods are able to outperform the AR model. Finally, Tian et al. (2017b) use Soybean, Cotton, Gluten Wheat, Corn, Early Indica Rice and Palm futures prices, traded in the Chinese market, to construct and forecast their realized volatility measure. Furthermore, the authors use several other realized volatility measures (such as daily log-range volatility, realized threshold multi-power variation and the realized threshold bi-power variation) and the jump component, as potential predictors of the realized volatility. Their predictive models allow both predictors and coefficients to vary over time. Their findings show that the Dynamic Model Average and Bayesian Model Average models are able to exhibit superior predictive ability, relatively to the simple HAR model. More importantly, they show that the HAR model with time-varying sparsity produces the most accurate forecasts for all the chosen commodities.

Given the limited research efforts on agricultural price volatility forecasting as well as the importance of such forecasts, it is imperative to further extend this line of research. Currently, the limited number of studies have not considered three rather important issues when it comes to agricultural commodities volatility forecasting. First, all previous papers use data from the Chinese futures markets, whereas there are no efforts to forecast volatility of agricultural commodities traded in the U.S., which is the most established market as well as the market with the highest penetration to both speculators and hedgers ${ }^{\mathbb{m}}$. Second, the main focus has been on realized volatility forecasting, whereas other intra-day volatility measures have been ignored. Finally, the current literature focuses on the aggregation of the information of agricultural commodities volatility (through bagging, combination

\footnotetext{
${ }^{1}$ See for example Bloomberg (2019).
} 
techniques, or time-varying approaches); nevertheless, they do not provide an answer as to whether specific volatility components, such as the jump component, the continuous component, the signed jumps, and the volatility or return leverage can provide better forecasts than simple HAR models. Thus, this study fills these voids and provides clear evidence as to whether the aforementioned components can provide predictive gains. This is rather important, given that complexity to forecasting models should only be added if this provide material predictive gains.

Succinctly, we add to this extremely scarce strand in the literature by applying several HAR-type models that accommodate the jump and continuous component, the signed jumps, and the volatility or return leverage (namely the HAR-J, HAR-CJ, HAR-PS and LHAR-CJ) to forecast different realized volatility measures (such as the realized volatility $R V$ and the median realized volatility $M e d R V$ ). For this study we focus on five important agricultural commodities traded in the Chicago Mercantile Exchange (CME) and the Intercontinental Exchange (ICE), namely, Corn, Rough Rice, Soybeans, Sugar, and Wheat and we produce forecasts for 1-day to 66-days ahead.

The choice of the $R V$ and $M e d R V$ volatility measures stems from the fact that the former is the most well-known volatility measure within past research but also among practitioners, whereas the latter is a more robust measure, compared to multipower variations, as large absolute returns associated with jumps tend to be eliminated from the calculation by the median operators. In addition, the $M e d R V$ offers a number of advantages over alternative measures of integrated variance in the presence of infrequent jumps and it is less sensitive to the presence of occasional zero intra-day returns (Theodosiou \& Zikes, 2011).

Our in-sample analysis shows that variants of the HAR model which decompose the volatility measure in its continuous path and jump component and take the volatility or return leverage effects into consideration (and in particular the LHAR-CJ model) are capable of offering better fit of the predictive equation for both the $R V$ and $M e d R V$ volatility measures. Turning to the out-of-sample results, these strongly suggest that the simple HAR model significantly outperforms the Random Walk and AR models. However, contrary to the in-sample findings, none of the HAR extensions is able to generate forecasts that are statistically significantly better compared to the simple HAR model. Hence, we cannot support the view that the decomposition of the volatility measure into its continuous path and jump component or even by taking into consideration the volatility or return leverage effect in a HAR-type model adds any incremental predictive accuracy. These results hold for both the $R V$ and $M e d R V$, hence the results are not volatility measure specific. Finally, we show that all HAR models have a marginally better directional accuracy compared to the random walk and AR models for the shorter forecasting horizons.

The remainder of this study is structured as follows. Section $\square$ describes the construction of the volatility measures, the predictive models and the loss functions for the 
forecast evaluations. Section 3 presents the data and their descriptive statistics. Section ⿴囗十 presents the results followed by a thorough discussion of the in-sample and real out-ofsample evaluation. Section 5 discusses the results from a risk management application. Finally, Section 6 concludes the study and provides avenues for further research.

\section{Methodology}

\subsection{Realized Variance measures and jump detection}

Let the number of intraday observations be $m$ and the total number of observation days be $M$. Intraday returns are then defined as log-difference of two consecutive prices

$$
r_{t, i}=\left(\log P_{t, i}-\log P_{t, i-1}\right) * 100,
$$

at day $t=1, \ldots, M$ for $i=2, \ldots, m$. The realized volatility of a given day $t$ is then defined as

$$
R V_{t}=\sum_{i=1}^{m} r_{t, i}^{2}
$$

Following Andersen \& Bollerslev (1998) and under the assumption of no serial correlation and other noise $\mathrm{I}^{\boldsymbol{\nabla}}$ in this discrete return data sampling, it holds that

$$
\operatorname{p}_{m \rightarrow \infty}\left(\int_{0}^{1} \sigma_{t+\tau}^{2} \mathrm{~d} \tau-\sum_{i=1}^{m} r_{t, i}^{2}\right)=0,
$$

where the integral describes the daily, continuous time volatility and the sum is the estimator of the daily realized volatility.

Discretizing data by equidistant sampling, where Eq. (3) does not hold any longer, might introduce intra-day price jumps which translate to higher realized variances. In order to have a more robust measure of realized volatility, Barndortt-Nielsen \& Sheppard (2004) introduce the concept of the bi-power variation $\left(B P V_{t}\right)$ which is defined as

$$
B P V_{t}=\frac{\pi}{2}\left(\frac{m}{m-1}\right) \sum_{j=1}^{m-1}\left|r_{t, j}\right|\left|r_{t, j+1}\right|
$$

This bi-power variation is being used to separate the realized variance in a continuous and discontinuous (jump) part. We use the approach of Huang (2004) to identify the jump component

$$
J_{t}=I_{\left\{Z_{t}>\Phi_{\alpha}\right\}}\left(R V_{t}-B P V_{t}\right)
$$

\footnotetext{
${ }^{2}$ We use tick-data of 5 -minute price intervals to circumvent some of the microstructure issues.
} 
where $\Phi(\cdot)$ refers to the density of a Standard Normal distribution with excess value

$$
Z_{t}=\sqrt{m} \frac{1-B P V_{t} \cdot R V_{t}^{-1}}{\sqrt{\left(\mu_{1}^{-4}+2 \mu_{1}^{-2}-5\right) \max \left(1, T Q_{t} \cdot B P V_{t}^{-2}\right)}}
$$

and $\mu_{1}=\mathbb{E}(Z)=\sqrt{2 / \pi}$. The tri-power quarticity $T Q_{t}$ is defined as

$$
T Q_{t}=m \mu_{4 / 3}^{-3} \sum_{j=1}^{m-2}\left|r_{t, j}\right|^{4 / 3}\left|r_{t, j+1}\right|^{4 / 3}\left|r_{t, j+2}\right|^{4 / 3}
$$

where $\mu_{p}=2^{p / 2} \cdot \Gamma(1 / 2 \cdot(p+1)) \cdot \Gamma(1 / 2)$. We set $\alpha=0.99$. The continuous component $C_{t}$ is then calculated as

$$
C_{t}=I_{\left\{Z_{t}>\Phi_{\alpha}\right\}} B P V_{t}+I_{\left\{Z_{t} \leq \Phi_{\alpha}\right\}} R V_{t}
$$

As the $B P V_{t}$ is not free of flaws, e.g. a downward-bias if there are zero-return ticks, an alternative is introduced by Andersen et al. (2012). This median realized volatility $M e d R V_{t}$ is defined as

$$
\operatorname{MedR} V_{t}=\frac{\pi}{6-4 \sqrt{3}+\pi} \frac{m}{m-2} \sum_{j=2}^{m-1} \operatorname{median}\left(\left|r_{t, j-1}\right|,\left|r_{t, j}\right|,\left|r_{t, j+1}\right|\right)^{2}
$$

which yields alternative continuous and jump components

$$
\begin{aligned}
& J_{t, \alpha}^{M e d R V}=I_{\left\{Z_{t}^{M e d R V_{t}}>\Phi_{\alpha}\right\}}\left(R V_{t}-M e d R V_{t}\right), \text { and } \\
& C_{t}^{M e d R V}=I_{\left\{Z_{t}>\Phi_{\alpha}\right\}} M e d R V_{t}+I_{\left\{Z_{t} \leq \Phi_{\alpha}\right\}} R V_{t}
\end{aligned}
$$

with

$$
\begin{aligned}
Z_{t}^{M e d R V} & =\sqrt{m} \frac{1-M e d R V_{t} \cdot R V_{t}^{-1}}{\sqrt{0.96 \max \left(1, M e d R Q_{t} \cdot M e d R V_{t}^{-2}\right)}}, \\
M e d R Q_{t} & =\frac{3 \pi}{9 \pi+72-52 \sqrt{3}} \frac{m}{m-2} \sum_{j=2}^{m-1} \operatorname{median}\left(\left|r_{t, j-1}\right|,\left|r_{t, j}\right|,\left|r_{t, j+1}\right|\right)^{4} .
\end{aligned}
$$

In order to further disaggregate realized volatilities to account for asymmetries, we also apply realized semi-variances which are based on Barndorff-Nielsen et al. (2010) and Patton \& Sheppard (2015)

$$
\begin{aligned}
& R S_{t}^{+}=\sum_{j=1}^{m} I_{\left\{r_{t, j}>0\right\}} r_{t, j}^{2}, \\
& R S_{t}^{-}=\sum_{j=1}^{m} I_{\left\{r_{t, j}<0\right\}} r_{t, j}^{2},
\end{aligned}
$$


and it naturally holds that $R V_{t}=R S_{t}^{+}+R S_{t}^{-}$.

\section{2. $R V$ Models}

In this section, we present the forecasting models for the realized volatility $(R V)$, whereas by replacing the $R V$ with the $M e d R V$, we can obtain the equivalent predictive models for the latter volatility measure. We follow the formulations of Corsi \& Renò (2012). Thus, for each forecasting horizon $h$ and each forecasting model we generate a different regression estimation. Doing so allows us to circumvent the use of recursive long-term forecasts based on the relative weights for 1-day ahead predictions for $h>1$. In particular, we define

$$
\begin{aligned}
& \log \left(R V_{t+h}^{(h)}\right)=\frac{1}{h} \sum_{j=1}^{h} \log \left(R V_{t+h-j+1}\right) \quad \text { and } \\
& \log \left(R V_{t}^{(h)}\right)=\frac{1}{h} \sum_{j=1}^{h} \log \left(R V_{t-j+1}\right),
\end{aligned}
$$

where $h \in\{1, \ldots, 66\}$ denotes the days ahead forecasting horizons. Note that, $\log \left(R V_{t+h}^{(h)}\right)$ is the average realized volatility for time $t+1$ to $t+h, \log \left(R V_{t}^{(h)}\right)$ is the average realized volatility for time $t-h+1$ to $t$, while $\log \left(R V_{t}\right)$ is the realized volatility at time $t$ and equivalent to $\log \left(R V_{t}^{(1)}\right)$.

As a baseline estimation, we use a simple Random Walk (RW), defined as:

$$
\log \left(R V_{t+h}^{(h)}\right)=\log \left(R V_{t}^{(h)}\right)+\varepsilon_{t+h}^{(h)},
$$

a simple autoregressive model of order one $(\mathrm{AR}(1))$, defined as:

$$
\log \left(R V_{t+h}^{(h)}\right)=\beta_{0}^{(t)}+\beta_{1}^{(t)} \log \left(R V_{t}^{(h)}\right)+\varepsilon_{t+h}^{(h)},
$$

as well as an autoregressive moving average model of order one $(\operatorname{ARMA}(1,1))$, defined as:

$$
\log \left(R V_{t+h}^{(h)}\right)=\beta_{0}^{(t)}+\beta_{1}^{(t)} \log \left(R V_{t}^{(h)}\right)+\beta_{2}^{(t)} \varepsilon_{t}^{(h)}+\varepsilon_{t+h}^{(h)}
$$

Subsequent to the three aforementioned naive and simple models, we focus on the standard HAR model of Corsi (200.9) and a number of extensions. The standard HAR model reads as follows:

$$
\log \left(R V_{t+h}^{(h)}\right)=\beta_{0}^{(t)}+\beta_{1}^{(t)} \log \left(R V_{t}\right)+\beta_{2}^{(t)} \log \left(R V_{t}^{(5)}\right)+\beta_{3}^{(t)} \log \left(R V_{t}^{(22)}\right)+\varepsilon_{t+h}^{(h)},
$$

where the $R V_{t}$ denotes the previous day's volatility, $R V_{t}^{(5)}$ denotes the averaged volatility during the previous week and finally, $R V_{t}^{(22)}$ denotes the averaged volatility over the

\footnotetext{
${ }^{3}$ As pointed out by Ederington \& Guan (20I0), the recursive forecast procedure introduces a bias to longer-term forecasts.
} 
previous month.

Next, in order to account for possible jumps, we augment the standard HAR with the simple jump measure $J_{t}$ to define the HAR-J model:

$$
\log \left(R V_{t+h}^{(h)}\right)=\beta_{0}^{(t)}+\beta_{1}^{(t)} \log \left(R V_{t}\right)+\beta_{2}^{(t)} \log \left(R V_{t}^{(5)}\right)+\beta_{3}^{(t)} \log \left(R V_{t}^{(22)}\right)+\beta_{4}^{(t)} \log \left(J_{t}+1\right)+\varepsilon_{t+h}^{(h)}
$$

Andersen et al. (20107) further propose to make use of bi-power variations to separate realized volatilites in a continuous and jump components, which we subsequently labeled HAR-CJ:

$$
\begin{aligned}
\log \left(R V_{t+h}^{(h)}\right)= & \beta_{0}^{(t)}+\beta_{1}^{(t)} \log \left(J_{t}+1\right)+\beta_{2}^{(t)} \log \left(J_{t}^{(5)}+1\right)+\beta_{3}^{(t)} \log \left(J_{t}^{(22)}+1\right) \\
& +\beta_{4}^{(t)} \log \left(C_{t}\right)+\beta_{5}^{(t)} \log \left(C_{t}^{(5)}\right)+\beta_{6}^{(t)} \log \left(C_{t}^{(22)}\right)+\varepsilon_{t+h}^{(h)} .
\end{aligned}
$$

In analogy to the definition of $R V_{t}^{(h)}$ above, we define $\log \left(C_{t}^{(h)}\right)=\frac{1}{h} \sum_{j=1}^{h} \log \left(C_{t-j+1}\right)$ and $J_{t}^{(h)}=\sum_{j=1}^{h} J_{t-j+1}$. Note that jumps are aggregated, not averaged.

The next model is one of the HAR specifications outlined in Patton \& Sheppard (2015) who separate realized volatilites in semi-variances to include measures for positive and negative daily log-returns $\left(r_{t}\right)$ as well as possible leverage effects. This model is labeled HAR-PS:

$$
\begin{aligned}
\log \left(R V_{t+h}^{(h)}\right)= & \beta_{0}^{(t)}+\beta_{1}^{(t)} \log \left(R S_{t}^{+}\right)+\beta_{2}^{(t)} \log \left(R S_{t}^{-}\right)+\beta_{3}^{(t)} I_{\left\{r_{t}<0\right\}} \log \left(R V_{t}\right) \\
& +\beta_{4}^{(t)} \log \left(R V_{t}^{(5)}\right)+\beta_{5}^{(t)} \log \left(R V_{t}^{(22)}\right)+\varepsilon_{t+h}^{(h)} .
\end{aligned}
$$

Finally, we use a leverage variant of the HAR-CJ, which is proposed by Corsi \& Renò (2012). This model separates the aggregated negative daily log-returns over the corresponding periods to account for leverage effects. The LHAR-CJ reads:

$$
\begin{aligned}
\log \left(R V_{t+h}^{(h)}\right)= & \beta_{0}^{(t)}+\beta_{1}^{(t)} \log \left(J_{t}+1\right)+\beta_{2}^{(t)} \log \left(J_{t}^{(5)}+1\right)+\beta_{3}^{(t)} \log \left(J_{t}^{(22)}+1\right) \\
& +\beta_{4}^{(t)} \log \left(C_{t}\right)+\beta_{5}^{(t)} \log \left(C_{t}^{(5)}\right)+\beta_{6}^{(t)} \log \left(C_{t}^{(22)}\right) \\
& +\beta_{7}^{(t)} r_{t}^{-}+\beta_{8}^{(t)} r_{t}^{(5)-}+\beta_{9}^{(t)} r_{t}^{(22)-}+\varepsilon_{t+h}^{(h)},
\end{aligned}
$$

with

$$
r_{t}^{(h)-}=\frac{1}{h} I_{\left\{\left(r_{t}+\ldots+r_{t-h+1}\right)<0\right\}}\left(r_{t}+\ldots+r_{t-h+1}\right) .
$$

The choice of the HAR model and its extensions is motivated by the fact that the existing literature has convincingly shown that this model class is the most appropriate framework to model and forecast intra-day volatility (such as the realized volatility and the median realized volatility). This has been shown not only for agricultural commodities (Tian et al, 2017a,b, Yang et al., 2017), but also for other commodities, such as crude oil, copper, and aluminum as well as stock market indices (Corsi \& Renò, 2012, Sévil, 2014, Zhang, 2017, Degiannakis \& Filis, 2017). 


\subsection{Forecasting E Evaluation}

As outlined in Section 2.2, we use different regression models for each forecasting horizon $h$. For example, when estimating the HAR model for $h=66$, i.e. $\log \left(R V_{t+66}^{(66)}\right)$, we obtain a prediction for the average $R V$ for the next 66 days and we use it as an estimate for the realized volatility in 66 days. Doing so allows us firstly, to circumvent any iterative forecasting procedure and secondly, to use the one-day ahead prediction for each model regardless of the forecasting horizon $h$. The idea is directly taken from Corsi \& Renò (2012).

To evaluate our forecasting results from the presented models over the $h$-days ahead horizons, for $h=1,5, \ldots, 66$, we employ three widely used loss functions, namely the Mean Squared Prediction Error (MSPE), the Mean Absolute Percentage Error (MAPE), and the QLIKE (Patton, 201T):

$$
\begin{gathered}
M S P E=\sqrt{N^{-1} \sum_{t=1}^{N}\left(R V_{t}-\widehat{R V_{t}}\right)^{2},} \\
M A P E=N^{-1} \sum_{t=1}^{N} \frac{\left|R V_{t}-\widehat{R V}_{t}\right|}{R V_{t}}, \\
Q L I K E=N^{-1} \sum_{t=1}^{N}\left(\log \left(\widehat{R V_{t}}\right)+\frac{\widehat{R V_{t}}}{R V_{t}}\right),
\end{gathered}
$$

where $R V_{t}$ and $\widehat{R V}_{t}$ are the actual realized volatility and the forecasted $R V$, respectively, at the different forecasting horizons and $N$ is the number of real out-of-sample forecasts. The forecasting errors are then compared using the Model Confidence Set (MCS, Hansen et al, 2011). The MCS is built by iteratively comparing all forecasts under consideration, the set $\mathcal{M}^{0}$, and by creating a subset of models with statistically indistinguishable performance from the best model, $\mathcal{M}^{*}$. Here, the best model refers to the one with the lowest loss function (MSPE, MAPE, and QLIKE). Thus, all models belonging to the set $\overline{\mathcal{M}^{*}}$, which are not part of the MCS, are performing statistically worse than all models included in the MCS. Following Hansen et al. (20II), we calculate two MCS sets, the $\mathcal{M}_{90 \%}^{*}$ for $\alpha=10 \%$ and $\mathcal{M}_{75 \%}^{*}$ for $\alpha=25 \%$, i.e. we construct a larger set of models with a confidence level of $90 \%$ and a more restrictive subset of the best models at the cost of a lower confidence of $75 \%$. We use the $T_{R}$ statistic and 10000 bootstraps with a block length of 3 to calculate the MCS. ${ }^{\text {⿴囗十 }}$

Moreover, we evaluate the directional accuracy of the predicted $R V$. To this end, we

\footnotetext{
${ }^{4}$ Our code on the estimation and forecasting is based on the code provided by Andrew Patton (http: //public.econ.duke.edu/ ap172/). The calculations of the MCS are performed using the MFE MatLab toolbox of Kevin Sheppard available from his personal webpage https://www.kevinsheppard.com/MFE_ [loolbox. All estimations, forecasts, and calculations are carried out in Matlab 2018a using an Intel i7-7700 and 32GB RAM.
} 
calculate the Success Ratio $(S R)$ by

$$
S R=N^{-1} \sum_{t=1}^{N} \mathbf{I}_{R V_{t} \cdot \widehat{R V_{t}}>0}
$$

where $\mathbf{I}_{R V_{t} \cdot \widehat{R V_{t}}>0}$ is an indicator function which is one if $R V_{t} \cdot \widehat{R V_{t}}>0$ and zero otherwise.

Thus, the $S R$ displays the ratio of a model's success to correctly predict the directional movement of the actual time series. In order to obtain directions from non-negative volatility forecasts, we de-mean the actual realized volatility $R V_{t}$ and its forecast $\left(\widehat{R V_{t}}\right)$ by their corresponding overall mean beforehand. The $S R$ is then tested using the test statistic presented by Pesaran \& Timmermann (1992).

We should note here that the same loss functions and evaluation methods are utilized for the $M e d R V$ forecasts as well.

\section{Data}

Our data set consists of tick-by-tick prices of the most liquid front month futures contracts of Corn, Rough Rice, Soybean, Sugar, and Wheat, traded at the CME and ICE, sampled from January 4, 2010 to June 30, 2017. The period of time is dictated by the data availability of these futures contracts. In order to circumvent microstructure noise, we aggregate our data to 5-min prices; see also Andersen \& Bollerslev (1998), Degiannakis (2010), and Lim et al (2015). Subsequently, we obtain data on $M=1898$ trading days with a total number of intra-day prices ranging from $m_{\text {total }}=234798$ (Sugar) to $m_{\text {total }}=399190$ (Rice). For our in-sample analysis we use the full number of daily observations, whereas for the real out-of-sample forecasts we use the period January 4, 2010 to December 31, 2012 for our estimation period and the period January 2, 2013 to June 30, 2017 for the out-of-sample forecasts, based on a rolling window approach with fixed window length of 3 years (roughly 750 observations). We opt for a rolling window approach given its superior ability to capture changes in the market conditions, as suggested by Engle et al. (19.90), Degiannakis \& Filis (2017), and Degiannakis et al. (2018).

Table $\mathbb{W}$ provides an overview of the sampling times and data sources.

Descriptive statistics and test statistics of the Ljung-Box test for five, ten, and 22 lags (trading days), corresponding to the aggregation in the HAR-type models, are presented in Tables 2-6. We report statistics for the realized volatility $\left(R V_{t}\right)$, the discontinuous jump component $\left(J_{t}\right)$, and the continuous component $\left(C_{t}\right)$ according to definitions given

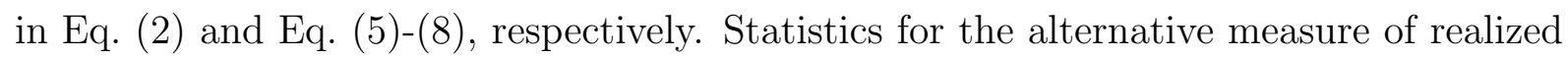

\footnotetext{
${ }^{5}$ Pesaran \& Timmermann ([1992) provide the test statistic $\frac{S R-S R^{*}}{\sqrt{\operatorname{var}(S R)-\operatorname{var}\left(S R^{*}\right)}} \stackrel{a}{\sim} N(0,1)$, where $S R^{*}=$ $P \cdot \widehat{P}+(1-P) \cdot(1-\widehat{P}), \operatorname{var}(S R)=S R^{*} \cdot\left(1-S R^{*}\right) / N, \operatorname{var}\left(S R^{*}\right)=(2 \widehat{P}-1)^{2} \cdot P \cdot(1-P) / N+(2 P-1)^{2}$. $\widehat{P} \cdot(1-\widehat{P}) / N+4 P \cdot \widehat{P} \cdot(1-P) \cdot(1-\widehat{P}) / N^{2}, P=N^{-1} \sum_{t=1}^{N} \mathbf{I}_{R V_{t}>0}$, and $\widehat{P}=N^{-1} \sum_{t=1}^{N} \mathbf{I}_{\widehat{R V}_{t}>0}$.
} 


\begin{tabular}{lcccc}
\hline Commodity & Exchange & Ticker & Sampling times (GMT) & Trading pauses (GMT) \\
\hline Corn & CBOT/CME & CN & Monday (01:00:05) - Friday (23:59:59) & $20: 01-22: 00$ \\
Rough Rice & CBOT/CME & RR & Monday (08:15:05) - Friday (23:59:59) & $20: 01-22: 00$ \\
Soybeans & CBOT/CME & SY & Monday (01:00:05) - Friday (23:59:59) & $20: 01-22: 00$ \\
Wheat & CBOT/CME & WC & Monday (11:00:05) - Friday (23:59:59) & $14: 01-17: 00$ \\
Sugar & ICE Futures U.S. & SB & Monday (05:31:00) - Friday (19:00:00) & - \\
\hline
\end{tabular}

Table 1: Overview of the acquired data, its source for each agricultural commodity futures, and sampling times.

volatility with the median RV measure, $M e d R V$, defined in Eq. (प), are given in the rightmost columns of those tables.

Sugar (Table 5) futures present the highest mean of realized volatilities at 3.8498 as well as the highest maximum daily volatility of 44.1071 which is almost twofold the second-highest value of the maximum of $R V_{t}$ (Wheat). Soybean (Table 四) shows the lowest values of mean and maximum of $R V_{t}$ as well as the lowest standard deviation. The statistics for Corn, Rough Rice, and Wheat are quite similar and less extreme than Sugar or Soybean. The results for the alternative measure of realized volatility, $M e d R V_{t}$, are qualitatively the same.

We find that for all five commodities, the measures for realized volatilities show significant autocorrelation on all lags, tested with the Ljung-Box test. This further motivates the application of autoregressive models such as the HAR and its extensions. Surprisingly, even the jump components $J_{t}$ for all commodities show autoregressive behaviour, indicating that agricultural commodity futures are indeed a special case if compared to high-frequency prices of crude oil or metal futures. Albeit with lower test statistics compared to its measures for realized volatilities, autocorrelated jump measures suggest that jumps in realized volatilities are a very common occurrence. We follow that high intraday price movements are the rule instead of an exception for agricultural prices in our sample period. This is supported by the relatively high Kurtosis of the realized volatility measures for all commodities. As the continuous component $C_{t}$ refers to the remaining realized volatility after removing jumps, the Ljung-Box test statistics are naturally much higher and take dimensions similar to $R V_{t}$. The findings for $M e d R V_{t}$ and its jump and continuous part decomposition are qualitatively the same. Since $M e d R V_{t}$ is more robust against small and high jumps compared to $R V_{t}$, we can expect a better forecasting performance given this highly volatile data set.

\begin{tabular}{l|cccccc}
\hline & $R V_{t}$ & $J_{t}$ & $C_{t}$ & $M e d R V_{t}$ & $J_{t}^{M e d R V}$ & $C_{t}^{M e d R V}$ \\
\hline Mean & 2.4523 & 0.2565 & 2.1958 & 2.1194 & 0.1880 & 2.2644 \\
Minimum & 0.2171 & 0.0000 & 0.0750 & 0.0905 & 0.0000 & 0.2171 \\
Maximum & 18.4265 & 5.9690 & 16.4022 & 13.2742 & 6.4750 & 16.4022 \\
StD & 1.7878 & 0.5188 & 1.6563 & 1.4810 & 0.5805 & 1.6324 \\
Skewness & 2.7020 & 4.5461 & 2.6810 & 2.7628 & 5.3108 & 2.8505 \\
Kurtosis & 14.2612 & 34.5191 & 14.0081 & 14.4542 & 39.8801 & 15.4021 \\
\hline Q(5) & $1987.95^{* * *}$ & $18.83^{* * *}$ & $1883.92^{* * *}$ & $1955.56^{* * *}$ & $24.07^{* * *}$ & $1818.43^{* * *}$ \\
Q(10) & $3119.27^{* * *}$ & $23.68^{* * *}$ & $3009.55^{* * *}$ & $3047.09^{* * *}$ & $45.80^{* * *}$ & $2854.76^{* * *}$ \\
Q(22) & $4696.17^{* * *}$ & $47.82^{* * *}$ & $4686.03^{* * *}$ & $4564.83^{* * *}$ & $96.88^{* * *}$ & $4318.17^{* * *}$ \\
\hline
\end{tabular}

Table 2: Descriptive statistics for Corn, sampled from January 4, 2010 to June 30, 2017 with $M=1898$ trading days and a total number of $m_{\text {total }}=399114$ prices at the 5 minutes interval.

Fig. $\mathbb{W}$ visualizes two measures of realized volatility $\left(R V_{t}\right.$ and $\left.M e d R V_{t}\right)$ and the jump measure $\left(J_{t, \alpha}\right)$ for Corn, Rough Rice, Soybean, Sugar, and Wheat in our sample period 


\begin{tabular}{l|cccccc}
\hline & $R V_{t}$ & $J_{t}$ & $C_{t}$ & MedRVt & $J_{t}^{M e d R V}$ & $C_{t}^{M e d R V}$ \\
\hline Mean & 3.1809 & 1.3842 & 1.7967 & 1.8069 & 0.9439 & 2.2370 \\
Minimum & 0.1245 & 0.0000 & 0.0158 & 0.0040 & 0.0000 & 0.0040 \\
Maximum & 19.7251 & 10.1585 & 18.6148 & 17.2740 & 11.1076 & 18.6148 \\
StD & 2.6145 & 1.4413 & 1.9326 & 1.7684 & 1.4327 & 2.2589 \\
Skewness & 2.0028 & 2.1103 & 2.8934 & 2.6134 & 2.4035 & 2.4388 \\
Kurtosis & 8.5347 & 9.0724 & 16.1702 & 13.9032 & 10.5472 & 11.5642 \\
\hline Q(5) & $1395.49^{* * *}$ & $349.95^{* * *}$ & $737.04^{* * *}$ & $940.26^{* * *}$ & $69.50^{* * *}$ & $799.13^{* * *}$ \\
$\mathrm{Q}(10)$ & $2486.75^{* * *}$ & $632.27^{* * *}$ & $1332.10^{* * *}$ & $1687.71^{* * *}$ & $101.03^{* * *}$ & $1474.60^{* * *}$ \\
$\mathrm{Q}(22)$ & $4434.15^{* * *}$ & $1137.62^{* * *}$ & $2352.03^{* * *}$ & $3050.09^{* * *}$ & $169.53^{* * *}$ & $2693.43^{* * *}$ \\
\hline
\end{tabular}

Table 3: Descriptive statistics for Rough Rice, sampled from January 4, 2010 to June 30, 2017 with $M=1898$ trading days and a total number of $m_{\text {total }}=399190$ prices at the 5 minutes interval.

\begin{tabular}{l|cccccc}
\hline & $R V_{t}$ & $J_{t}$ & $C_{t}$ & $M e d R V_{t}$ & $J_{t}^{M e d R V}$ & $C_{t}^{M e d R V}$ \\
\hline Mean & 1.5387 & 0.1475 & 1.3912 & 1.2902 & 0.1471 & 1.3916 \\
Minimum & 0.0201 & 0.0000 & 0.0043 & 0.0019 & 0.0000 & 0.0019 \\
Maximum & 8.4025 & 5.4881 & 8.2409 & 10.7046 & 5.2553 & 8.2409 \\
StD & 1.0307 & 0.3581 & 0.9419 & 0.8652 & 0.4306 & 0.9196 \\
Skewness & 2.4411 & 6.0922 & 2.4766 & 2.9417 & 5.8652 & 2.5342 \\
Kurtosis & 11.4026 & 61.5027 & 12.1233 & 18.7540 & 50.2153 & 12.9593 \\
Q(5) & $1680.15^{* * *}$ & $22.01^{* * *}$ & $1976.96^{* * *}$ & $2052.24^{* * *}$ & $16.96^{* * *}$ & $2172.94^{* * *}$ \\
Q(10) & $2479.40^{* * *}$ & $29.62^{* * *}$ & $2916.09^{* * *}$ & $3037.15^{* * *}$ & $19.63^{* * *}$ & $3234.40^{* * *}$ \\
Q(22) & $3581.27^{* * *}$ & $43.12^{* * *}$ & $4120.91^{* * *}$ & $4289.34^{* * *}$ & $33.76^{* * *}$ & $4518.27^{* * *}$ \\
\hline
\end{tabular}

Table 4: Descriptive statistics for Soybean, sampled from January 4, 2010 to June 30, 2017 with $M=1898$ trading days and a total number of $m_{\text {total }}=399126$ prices at the 5 minutes interval.

\begin{tabular}{l|cccccc}
\hline & $R V_{t}$ & $J_{t}$ & $C_{t}$ & MedRV & $J_{t}^{M e d R V}$ & $C_{t}^{M e d R V}$ \\
\hline Mean & 3.8498 & 0.2875 & 3.5623 & 3.2655 & 0.3095 & 3.5403 \\
Minimum & 0.2853 & 0.0000 & 0.2665 & 0.3312 & 0.0000 & 0.2853 \\
Maximum & 44.1071 & 5.3501 & 44.1071 & 46.5043 & 6.5751 & 44.1071 \\
StD & 3.1993 & 0.6453 & 3.0801 & 2.8760 & 0.8004 & 3.0541 \\
Skewness & 3.2517 & 3.1877 & 3.5814 & 4.3797 & 3.5174 & 3.6207 \\
Kurtosis & 24.5723 & 15.5835 & 28.9848 & 43.1246 & 17.7357 & 29.5813 \\
Q(5) & $3260.05^{* * *}$ & $22.47^{* * *}$ & $3081.98^{* * *}$ & $2775.52^{* * *}$ & $34.32^{* * *}$ & $2987.32^{* * *}$ \\
Q(10) & $5641.97^{* * *}$ & $42.31^{* * *}$ & $5320.00^{* * *}$ & $4792.44^{* * *}$ & $56.22^{* * *}$ & $5193.60^{* * *}$ \\
Q(22) & $10486.96^{* * *}$ & $107.35^{* * *}$ & $9812.76^{* * *}$ & $8760.67^{* * *}$ & $119.38^{* * *}$ & $9588.06^{* * *}$ \\
\hline
\end{tabular}

Table 5: Descriptive statistics for Sugar, sampled from January 4, 2010 to June 30, 2017 with $M=1898$ trading days and a total number of $m_{\text {total }}=234798$ prices at the 5 minutes interval.

\begin{tabular}{l|cccccc}
\hline & $R V_{t}$ & $J_{t}$ & $C_{t}$ & $M e d R V_{t}$ & $J_{t}^{M e d R V}$ & $C_{t}^{M e d R V}$ \\
\hline Mean & 3.2421 & 0.3139 & 2.9282 & 2.7216 & 0.2813 & 2.9608 \\
Minimum & 0.1040 & 0.0000 & 0.1040 & 0.0601 & 0.0000 & 0.1040 \\
Maximum & 26.0959 & 6.4052 & 26.0959 & 24.4544 & 6.7609 & 26.0959 \\
StD & 2.2858 & 0.6015 & 2.1958 & 1.9768 & 0.6948 & 2.1531 \\
Skewness & 2.8804 & 3.3899 & 3.1174 & 3.2825 & 3.6660 & 3.1371 \\
Kurtosis & 17.5920 & 21.0279 & 20.2421 & 22.2619 & 20.6146 & 20.9127 \\
Q(5) & $2343.74^{* * *}$ & $48.49^{* * *}$ & $2104.90^{* * *}$ & $2076.85^{* * *}$ & $71.31^{* * *}$ & $2124.42^{* * *}$ \\
Q(10) & $3413.07^{* * *}$ & $66.45^{* * *}$ & $3022.29^{* * *}$ & $3007.03^{* * *}$ & $103.52^{* * *}$ & $3007.07^{* * *}$ \\
Q(22) & $5421.44^{* * *}$ & $101.57^{* * *}$ & $4755.90^{* * *}$ & $4833.95^{* * *}$ & $162.93^{* * *}$ & $4705.53^{* * *}$ \\
\hline
\end{tabular}

Table 6: Descriptive statistics for Wheat, sampled from January 4, 2010 to June 30, 2017 with $M=1898$ trading days and a total number of $m_{\text {total }}=399114$ prices at the 5 minutes interval.

January 4, 2010 to June 30, 2017. Interestingly enough we show that the two volatility measures are closely related yet there are certain peaks, especially in the case of Rice, that are not observed for both measures. This is due to the fact that the $M e d R V$ measure is more robust against jumps. Similarly, the jump component behaves rather differently for the different commodities, with a common feature that fewer jumps are apparent during 2013-2014.

\footnotetext{
${ }^{6}$ We note that the daily data for the $R V, M e d R V$, and their jump components are available upon request by the authors.
} 

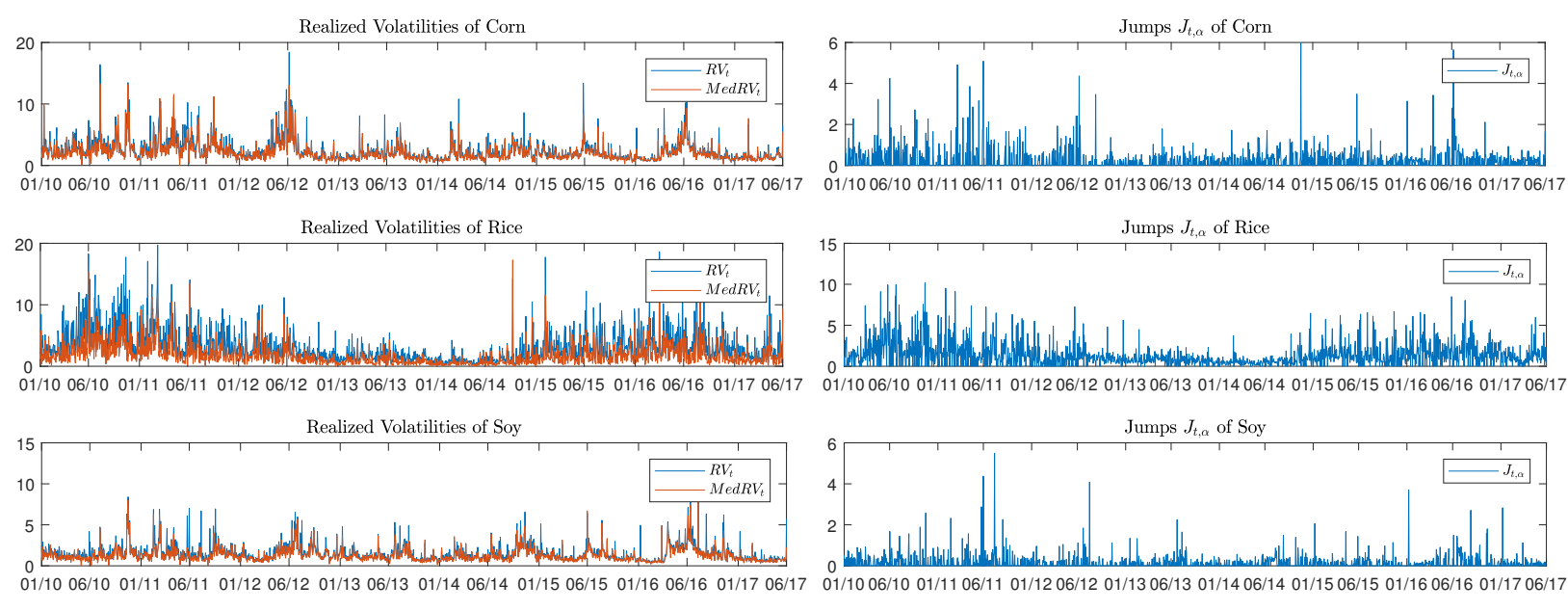

Realized Volatilities of Suga
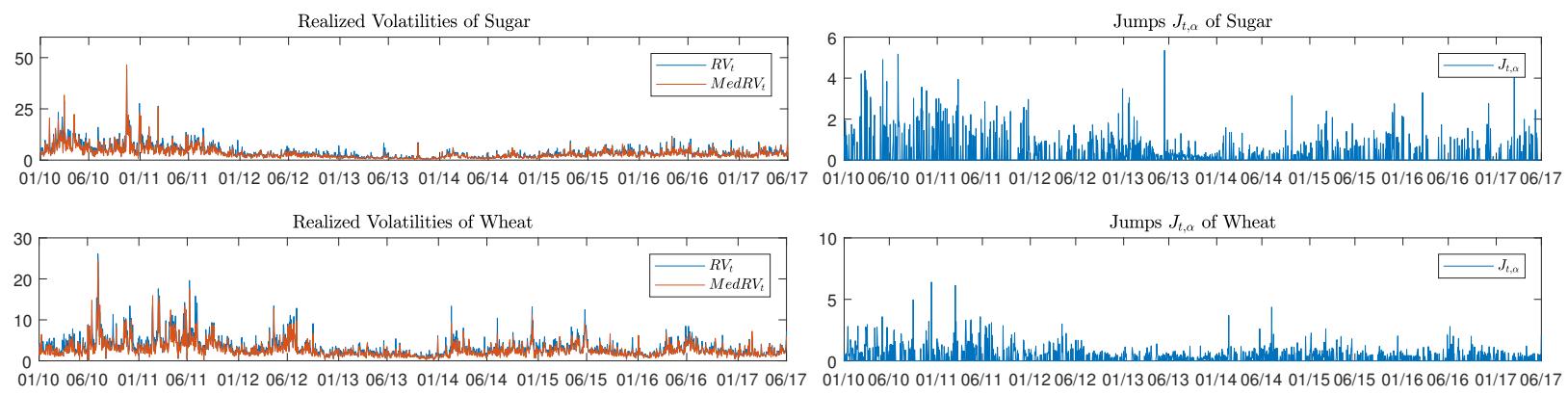

Figure 1: Realized volatility measures $\left(R V_{t}\right.$ and $\left.M e d R V_{t}\right)$ and jump measure $\left(J_{t, \alpha}\right)$ for Corn, Rough Rice, Soybean, Sugar, and Wheat for the sample period January 4, 2010 to June 30, 2017.

\section{Results \& Discussion}

\subsection{In-Sample Results}

Our in-sample results are presented in Tables [-[1] for $R V$ and in Tables A.17-A.2] (in Appendix A) for $M e d R V$, given that the results are qualitatively similar for both volatility measures. Each table shows the parameter estimates as well as loss function for all seven models over all five forecasting horizons.

The best model over all commodities and horizons appears to be the LHAR-CJ, which consistently has the highest $R^{2}$ and, with a few exemptions, the lowest loss functions, i.e. it always belongs to the $\mathcal{M}_{75 \%}^{*}$. Comparing the class of HAR models with the naive Random Walk and the AR(1), we conclude that, except for a few instances, the HAR models are superior with regards to model fit. This reveals the fact that a long-term component in the volatility helps to explain the variance of the volatility. The high $t$-statistics for the $R V$ and $C$ parameters with 5 and 22 days support this assessment. For the Random Walk with forecasting horizons greater than one day, we notice that it performs even worse than the sample mean which is depicted by negative $R^{2}$.

Another interesting observation is that the leverage effect appears weak. Thus, in the in-sample series of Corn, Rice, Soybean, Sugar, and Wheat the interaction term between dummy variable of a negative return and the RV is merely of statistical importance. For the negative return parameters in the LHAR-CJ model, we find that the lag of $r_{t}^{-}$ somewhat corresponds to the forecasting horizon $h$, i.e. for small forecasting horizons we 
observe higher $t$-statistics and decreasing $t$-statistics for higher horizons for the first lag. For the fifth and twenty-second lag, however, we find reverse behaviour. We presume that this association roots in the way the regression models for the different forecasting horizons are constructed, e.g. for $h=22$ the model forecasts the average volatility over 22 days and the leverage component for 22-days of average negative returns contains more information for this regression than the last leverage component for the preceding day.

A similar pattern is noticeable from the jump components in the HAR-CJ and LHARCJ models. Again, we see a correlated behavior of the components' lag with the forecasting horizon for most commodities. However, for the HAR-J, i.e. for the model with only one lagged jump, the statistical significance varies over the five commodities. While we observe slightly statistically significant parameters for Corn, Rice, Sugar, and Wheat over all horizons from 1-day to 66-days ahead, the parameter is not distinguishable from zero for Soybean. ${ }^{\square}$

From the in-sample analysis, we conclude that the best performing model is the most complex one: the LHAR-CJ depicting long memory, leverage effect, and a differentiation between continuous and jump components. Moreover, the importance of the lags of the leverage and the jump parameters appear to be positively associated with the forecasting horizon. Thus, we conclude that stylized facts are important to describe the in-sample volatility of agricultural commodities. The fact that LHAR-CJ includes all those components at different time horizons makes it consistently superior to its peer over all horizons.

\subsection{Real out-of-sample forecasting results}

From the in-sample evaluation we show that the LHAR-CJ is the best performing model for all agricultural commodities, across all horizons, and for both volatility measures. Nevertheless, to be able to generate solid conclusions we need to assess the performance of our models in real out-of-sample forecasts.

Thus, we turn our attention to the real out-of-sample forecasting evaluation based on the MSPE, MAPE, and QLIKE. Furthermore, we use the MCS test to identify the set of the best models with equal predictive accuracy. The results are depicted in Tables ए2-ए6 for $R V{ }^{\nabla}$ At a first glance, we notice that none of the competing models can consistently improve the forecast accuracy that we obtain from the simple HAR model.

More specifically, the Random Walk, AR, and ARMA models largely underperform compared to the HAR-type models under any loss function and for all commodities; although they are among the best performing models in the longer forecasting horizons for the $R V$ measure under specific loss function per commodity.

Turning to the HAR-type models, we observe that they significantly outperform the Random Walk and AR models based on the MCS test, except from the cases outlined earlier. The most important finding, though, is that the simple HAR model is not con-

\footnotetext{
${ }^{7}$ Note that this finding is only robust for higher horizons if we look at $R V$ measure.

${ }^{8}$ Results for $M e d R V$ are presented in Tables $4.22-4.26$ in Appendix A.
} 


\begin{tabular}{|c|c|c|c|}
\hline$h$ & 1 & 5 & 10 \\
\hline & & & indom Walk \\
\hline adj. $\mathrm{R}^{2}$ & 0.2291 & 0.4768 & 0.4601 \\
\hline MSPE & $\begin{array}{l}1.6701 \\
1.670\end{array}$ & $\begin{array}{l}.46512 \\
1.6512\end{array}$ & $1.6991^{* * *}$ \\
\hline MAPE & 0.4281 & 0.4417 & 0.4640 \\
\hline & 1.8564 & $1.8607^{* *}$ & $1.8720^{* *}$ \\
\hline & & & $\mathrm{AR}(1)$ \\
\hline $\begin{array}{l}\text { c } \\
R V^{(h)}\end{array}$ & $\begin{array}{c}0.2715 \\
(13.7709) \\
0.6144 \\
(26.6586)\end{array}$ & $\begin{array}{c}0.1828 \\
(14.1551) \\
0.7377 \\
(44.3740)\end{array}$ & $\begin{array}{c}0.1888 \\
(15.1709) \\
0.7296 \\
(44.8092)\end{array}$ \\
\hline adj. $\mathrm{R}^{2}$ & 0.3770 & 0.5452 & 0.5329 \\
\hline MSPI & 1.4961 & 1.6007 & $1.6591^{* *}$ \\
\hline MAPE & $\begin{array}{l}0.3763 \\
0\end{array}$ & $\begin{array}{c}0.4121^{*} \\
1838 * *\end{array}$ & $\begin{array}{l}0.4324^{*} \\
0.849^{* *}\end{array}$ \\
\hline QLIKE & $1.8214^{* *}$ & $1.8384^{* *}$ & $1.8489^{* *}$ \\
\hline & & & ARMA \\
\hline & 0.0361 & 0.0886 & 0.1202 \\
\hline & (4.4106) & $(7.4290)$ & (8.6521) \\
\hline$R V^{(h)}$ & $\begin{array}{c}0.9485 \\
(98.4321)\end{array}$ & $\begin{array}{c}0.8733 \\
(60.7486)\end{array}$ & $\begin{array}{c}0.8279 \\
(51.8560)\end{array}$ \\
\hline & $\begin{array}{c}(98.4321) \\
-0.6590\end{array}$ & $\begin{array}{l}(60.7486) \\
-0.3186\end{array}$ & $\begin{array}{c}(51.8500) \\
-0.2148\end{array}$ \\
\hline$\varepsilon^{(h)}$ & (31.1119) & (11.5396) & $(7.4564)$ \\
\hline & 0.4574 & 0.5645 & 0.5441 \\
\hline & $1.4134^{* *}$ & $\begin{array}{l}1.5866^{*} \\
0.404 * *\end{array}$ & $1.6557^{* *}$ \\
\hline $\begin{array}{l}\text { MAPE } \\
\text { QLIKE }\end{array}$ & $\begin{array}{l}0.3993^{* *} \\
1.8098^{* *}\end{array}$ & $\begin{array}{l}0.40453^{* *} \\
1.831^{*}\end{array}$ & $\begin{array}{l}0.4300^{* * *} \\
1.8483^{* *}\end{array}$ \\
\hline & & & HAR \\
\hline & 0.0769 & 0.1143 & 0.1485 \\
\hline c & (3.9033) & $(8.2035)$ & $(11.2609)$ \\
\hline$R V^{(1)}$ & $\begin{array}{l}0.2710 \\
(8.453\end{array}$ & $\begin{array}{l}0.1642 \\
(74855)\end{array}$ & $\begin{array}{l}0.1352 \\
6.4097\end{array}$ \\
\hline & $\begin{array}{c}(8.45937) \\
0.3677\end{array}$ & 0.3480 & $\begin{array}{c}(6.4997) \\
0.2780\end{array}$ \\
\hline$R V^{(5)}$ & (7.1703) & $\begin{array}{l}0.04000 \\
(9.9696)\end{array}$ & $(8.5140)$ \\
\hline$R V^{(22)}$ & 0.2507 & 0.3220 & 0.3723 \\
\hline & & & \\
\hline adj. $R^{2}$ & 0.4616 & 0.5829 & 0.5719 \\
\hline $\begin{array}{l}\text { MSPE } \\
\text { MAPE }\end{array}$ & $\begin{array}{l}1.4138^{* * *} \\
0.344^{* *}\end{array}$ & $\begin{array}{l}1.5823 \\
0.4036^{* * *}\end{array}$ & $\begin{array}{l}1.6542^{* * *} \\
0.4299^{*}\end{array}$ \\
\hline QLIKE & $1.8094^{* *}$ & $1.8347^{* *}$ & $1.8475^{* *}$ \\
\hline & & & HAR-J \\
\hline c & 0.0803 & 0.1228 & 0.1562 \\
\hline & $\begin{array}{c}(3.8114) \\
0.2767\end{array}$ & $\begin{array}{c}(8.07883) \\
0.1788\end{array}$ & $\begin{array}{l}(10.9821) \\
0.1485\end{array}$ \\
\hline$R V^{(1)}$ & $(7.7882)$ & $(7.2318)$ & $\begin{array}{l}0.1480 \\
(6.3356)\end{array}$ \\
\hline$R V^{(5)}$ & $\begin{array}{l}0.3672 \\
(7.7720\end{array}$ & 0.3466 & 0.2768 \\
\hline & $(7.1720)$ & $(9.9612)$ & $(8.4759)$ \\
\hline$R V^{(22)}$ & $\begin{array}{c}0.2496 \\
(5.1870)\end{array}$ & $\begin{array}{l}0.3190 \\
(9.4131)\end{array}$ & $\begin{aligned} 0.3695 \\
(11.3890)\end{aligned}$ \\
\hline$J^{(1)}$ & $\begin{array}{l}(3.1870) \\
-0.0204\end{array}$ & $\begin{array}{l}(9.4111) \\
-0.0522\end{array}$ & $\begin{array}{c}(11.3890) \\
-0.0473\end{array}$ \\
\hline$J^{(1)}$ & $\begin{array}{c}-0.0204 \\
(-0.3689)\end{array}$ & $\begin{array}{l}(-0.0522 \\
(-1.3986)\end{array}$ & $(-1.4217)$ \\
\hline adj. $R^{2}$ & 0.4614 & 0.58 & 0.57 \\
\hline & $1.4137^{* * *}$ & $1.5804^{*}$ & $1.6531^{* * *}$ \\
\hline $\begin{array}{l}\text { MAPE } \\
\text { QLIKE }\end{array}$ & $\begin{array}{l}0.3475^{* *} \\
1.8095^{* *}\end{array}$ & $\begin{array}{l}0.4035^{* *} \\
1.8345^{* *}\end{array}$ & $\begin{array}{l}0.4296^{*} \\
1.8473^{* *}\end{array}$ \\
\hline & & & HAR-CJ \\
\hline c & 0.1979 & 0.2560 & 0.2925 \\
\hline & $\begin{array}{c}(4.4140) \\
0.1488\end{array}$ & $\begin{array}{l}(8.1272) \\
0.0859\end{array}$ & $\begin{array}{l}(10.0820) \\
0.0595\end{array}$ \\
\hline$J^{(1}$ & $(3.2641)$ & $\begin{array}{l}(2.8191) \\
\text { (2. }\end{array}$ & $\begin{array}{c}0.0595 \\
(2.1244)\end{array}$ \\
\hline$J^{(5)}$ & $\begin{array}{l}0.0730 \\
(2.3276)\end{array}$ & 0.0575 & 0.0326 \\
\hline$J^{(22)}$ & $\begin{array}{l}(2.3276) \\
-0.0291\end{array}$ & $\begin{array}{l}(2.6926) \\
-0.0381\end{array}$ & $\begin{array}{l}(1.6555) \\
-0.0331)\end{array}$ \\
\hline$J^{(22)}$ & $\begin{array}{r}-0.0291 \\
(-0.9481)\end{array}$ & $\begin{array}{l}-0.0381 \\
(-1.6990)\end{array}$ & $\begin{array}{r}-0.0331 \\
(-1.6055)\end{array}$ \\
\hline$C^{(1}$ & 0.2383 & 0.1439 & 0.1178 \\
\hline & $(7.8100)$ & $(6.9921)$ & $(6.1939)$ \\
\hline$C^{(5)}$ & $\begin{array}{c}0.3260 \\
(6.8119)\end{array}$ & $\begin{array}{l}0.3144 \\
(9.6263)\end{array}$ & $\begin{array}{c}0.2675 \\
(8.8319)\end{array}$ \\
\hline$C^{(22)}$ & 0.2685 & 0.3373 & 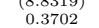 \\
\hline$C^{(22)}$ & $(5.6877)$ & $(9.5194)$ & (11.0729) \\
\hline adj. $\mathrm{R}^{2}$ & 0.4609 & 0.5863 & 0.5783 \\
\hline MSPE & $1.4159^{* *}$ & $1.5747^{* * *}$ & $1.6495^{* *}$ \\
\hline MAPE & $0.3480^{* *}$ & $0.4036^{* * *}$ & $0.4268^{* *}$ \\
\hline & $1.8089^{* *}$ & $1.8337^{* * *}$ & $1.8458^{* *}$ \\
\hline & & & HAR-PS \\
\hline c & $\begin{array}{l}0.2720 \\
(8.8286)\end{array}$ & 0.2315 & 0.2404 \\
\hline & $\begin{array}{c}(8.8286) \\
0.0985\end{array}$ & $\begin{array}{l}(10.35899) \\
0.0574\end{array}$ & $\begin{array}{l}(10.9672) \\
0.0376\end{array}$ \\
\hline$R S^{+}$ & $\begin{array}{l}0.0985 \\
(3.6287)\end{array}$ & $(3.1694)$ & $(2.1823)$ \\
\hline$R S^{-}$ & $\begin{array}{l}0.1751 \\
0\end{array}$ & 0.1069 & 0.0912 \\
\hline & $(5.0766)$ & $(4.4604)$ & $(3.8958)$ \\
\hline $\mathbf{I}_{r_{t}<0} R V^{(1)}$ & $\begin{array}{c}-0.0152 \\
(-0.5522)\end{array}$ & $\begin{array}{c}-0.0076 \\
(-0.3611)\end{array}$ & $\begin{array}{l}0.0010 \\
(0.0521)\end{array}$ \\
\hline$R V^{(5)}$ & $\begin{array}{c}0.3762 \\
0.3238\end{array}$ & 0.3537 & 0.2856 \\
\hline & (7.3238) & $(10.05$ & $(8.66)$ \\
\hline$R V^{(22)}$ & $\begin{array}{c}0.2516 \\
(5.2345)\end{array}$ & $\begin{array}{l}0.3226 \\
(9.5383)\end{array}$ & $\begin{array}{c}0.3726 \\
(11.4684)\end{array}$ \\
\hline adj. $R^{2}$ & 0.4593 & 0.5815 & 0.5709 \\
\hline & 1.415 & 1.58 & 1.65 \\
\hline & $0.3477^{* *}$ & $0.4040^{* *}$ & $0.4305^{*}$ \\
\hline QLIKE & $1.8096^{* *}$ & $1.8350^{* *}$ & $1.8479^{* *}$ \\
\hline & & & LHAR-CJ \\
\hline c & 0.1869 & 0.23 & 0.2741 \\
\hline & & & \\
\hline$J^{(1)}$ & $\begin{array}{c}0.1429 \\
(3.1675)\end{array}$ & 0.0806 & 0.0548 \\
\hline$I^{(t}$ & 0.0732 & 0.0574 & 0.0324 \\
\hline$J^{(25}$ & $(2.3617)$ & $(2.7158)$ & (1.6637) \\
\hline & -0.0382 & -0.0337 & -0.0 \\
\hline & $(-1.1599)$ & $(-1.4448)$ & $(-1.4$ \\
\hline$C$ & 0.2224 & 0.1352 & 0.1 \\
\hline & & & (5.7 \\
\hline$C^{(5)}$ & $\begin{array}{c}0.3880 \\
(6.8660)\end{array}$ & $\begin{array}{c}0.3064 \\
(9.3713)\end{array}$ & $\begin{array}{l}0.2632 \\
(8.6812)\end{array}$ \\
\hline$C^{(22)}$ & 0.2793 & 0.3413 & 0.3749 \\
\hline$C^{(22)}$ & $(5.9710)$ & $(9.6185)$ & (11.2086) \\
\hline$r_{t}^{-,(1)}$ & -0.0417 & -0.0225 & -0.0240 \\
\hline & $(-3.1915)$ & $(-2.5805)$ & $(-3.0373)$ \\
\hline , (5) & $\begin{array}{c}-0.0030 \\
(-10386\end{array}$ & $\begin{array}{c}0.0009 \\
(0.48322\end{array}$ & $\begin{array}{c}-0.0001 \\
-(-0.0528\end{array}$ \\
\hline & -0.0601 & -0.0789 & $\begin{array}{c}(-0.0068) \\
-0.0632\end{array}$ \\
\hline$r_{t}^{-,-(22)}$ & $(-1.1275)$ & $\begin{array}{l}-0.0898) \\
(-2.4668)\end{array}$ & $\begin{array}{l}-(-2.2226) \\
(-2.226\end{array}$ \\
\hline & & & \\
\hline & $1.4050^{* * *}$ & $1.5757^{* *}$ & $1.6520^{* * *}$ \\
\hline $\begin{array}{l}\text { MAPE } \\
\text { QLIKE }\end{array}$ & $\begin{array}{l}0.3453^{* *} \\
1.8079^{* *}\end{array}$ & $\begin{array}{l}0.4029^{9^{*}} \\
1.8336^{* *}\end{array}$ & $\begin{array}{l}0.4258^{* *} \\
1.8456^{* *}\end{array}$ \\
\hline
\end{tabular}

Table 7: In-Sample regression results for Corn with $R V$. Note that * and ** indicate the inclusion in the $\mathcal{M}_{90 \%}^{*}$ and $\mathcal{M}_{75 \%}^{*}$, respectively. The $t$-statistics for the parameter estimates are given in parentheses. 


\begin{tabular}{|c|c|c|c|}
\hline$h$ & 1 & 5 & 10 \\
\hline & & & ddom Walk \\
\hline adj. $R^{2}$ & 0.1138 & 0.5612 & 0.6559 \\
\hline $\begin{array}{l}\text { MSPE } \\
\text { MAPE }\end{array}$ & $\begin{array}{l}2.7428 \\
0.7216\end{array}$ & $\begin{array}{l}2.3759 \\
0.6141\end{array}$ & $\begin{array}{l}2.3925^{* *} \\
0.6208\end{array}$ \\
\hline QLIKE & 2.1942 & $2.1099^{* *}$ & $2.1159^{* *}$ \\
\hline & & & $\operatorname{AR}(1)$ \\
\hline c & $\begin{array}{c}0.3812 \\
(15.2813)\end{array}$ & $\begin{array}{c}0.1890 \\
(11.7710)\end{array}$ & $\begin{array}{c}0.1482 \\
(9.6476)\end{array}$ \\
\hline$R V^{(h)}$ & $\begin{array}{c}0.5567 \\
(26.5044)\end{array}$ & $\begin{array}{c}0.7807 \\
(53.6456)\end{array}$ & $\begin{array}{c}0.8281 \\
(57.8886)\end{array}$ \\
\hline adj. $R^{2}$ & 0.3093 & 0.6088 & 0.6851 \\
\hline MSPE & 2.3864 & 2.3566 & 2.3908 \\
\hline MAPE & 0.6134 & 0.5945 & $0.6074^{*}$ \\
\hline QLIKE & $2.1165^{* *}$ & $2.0991^{* *}$ & $2.1084^{* *}$ \\
\hline & & & ARMA \\
\hline & 0.0122 & 0.0364 & 0.0360 \\
\hline c & $(2.7418)$ & $(5.0655)$ & (5.0083) \\
\hline$R V^{(h)}$ & 0.9861 & 0.9584 & 0.9581 \\
\hline & (217.6556) & $(130.3651)$ & $(134.2256)$ \\
\hline$\varepsilon^{(h)}$ & $\begin{array}{l}-0.08835 \\
(59.7307)\end{array}$ & $\begin{array}{l}-0.5577 \\
(23.9696)\end{array}$ & $\begin{array}{l}-0.5131 \\
(21.1455)\end{array}$ \\
\hline & 0.4393 & 0.6644 & 0.7069 \\
\hline & $\begin{array}{l}0.25350 \\
2.2252\end{array}$ & 2.310 & 2.372 \\
\hline & 0.5399 & $0.5713^{* *}$ & $0.5949^{* *}$ \\
\hline QLIKE & $2.0656^{* *}$ & $2.0848^{* *}$ & $2.0996^{* *}$ \\
\hline & & & HAR \\
\hline c & 0.0730 & 0.0962 & 0.1138 \\
\hline & $\begin{array}{c}(2.8141) \\
0.1669\end{array}$ & $\begin{array}{l}(5.8371) \\
0.0724\end{array}$ & $\left.\begin{array}{l}(7.4777) \\
0.0611\end{array}\right)$ \\
\hline$R V^{(1)}$ & $\begin{array}{l}0.1009 \\
(5.7515)\end{array}$ & $(4.1456)$ & $\begin{array}{l}0.0011 \\
(3.9159)\end{array}$ \\
\hline$R V^{(5)}$ & $\begin{array}{c}0.2778 \\
(4.9183)\end{array}$ & 0.3124 & 0.3048 \\
\hline RV & $(4.9183)$ & $(9.5792)$ & (10.5476) \\
\hline$R V^{(22)}$ & $\begin{array}{c}0.4712 \\
(8.6123)\end{array}$ & $\begin{array}{c}0.5036 \\
(15.5121)\end{array}$ & $\begin{array}{c}0.5019 \\
(17.5319)\end{array}$ \\
\hline adj. $R^{2}$ & 0.4396 & 0.6576 & 0.7028 \\
\hline MSPE & 2.2208 & $2.3163^{*}$ & $2.3666^{* *}$ \\
\hline $\begin{array}{l}\text { MAPE } \\
\text { QLIKE }\end{array}$ & $\begin{array}{c}0.5375 \\
2.0651^{* *}\end{array}$ & $\begin{array}{l}0.5739^{* *} \\
2.0867^{* *}\end{array}$ & $\begin{array}{l}0.5939^{* *} \\
2.0979^{* *}\end{array}$ \\
\hline & & & HAR-J \\
\hline c & 0.1168 & 0.1137 & 0.1337 \\
\hline & $\begin{array}{c}(3.2765) \\
0.2117\end{array}$ & $\begin{array}{l}(5.3059) \\
0.0904\end{array}$ & $\begin{array}{c}(6.9838) \\
0.0815\end{array}$ \\
\hline$R V^{(1)}$ & $\begin{array}{l}0.2117 \\
(5.2178)\end{array}$ & $(3.7525)$ & $(3.9482)$ \\
\hline$R V^{(5)}$ & 0.2793 & 0.3130 & 0.3055 \\
\hline & $(4.9471)$ & $(9.5988)$ & (10.5656) \\
\hline$R V^{(22)}$ & $\begin{array}{l}0.4668 \\
(8.5367)\end{array}$ & $\begin{array}{c}0.5019 \\
(15.4413)\end{array}$ & $\begin{array}{c}0.4999 \\
(17.4639)\end{array}$ \\
\hline$J(1)$ & $\begin{array}{l}(8.5367) \\
-0.0974\end{array}$ & $\begin{array}{l}(1.0390) \\
(1.443)\end{array}$ & $\begin{array}{l}(7.0 .449) \\
-0.0444\end{array}$ \\
\hline$J^{(1)}$ & $\begin{array}{c}-0.0974 \\
(-1.6883)\end{array}$ & $(-1.2108)$ & $(-1.5683)$ \\
\hline adj. $R^{2}$ & 0.4402 & 0.6577 & 0.70 \\
\hline $\begin{array}{l}P E \\
P E\end{array}$ & 2.2182 & $2.3174^{*}$ & $2.3657^{* *}$ \\
\hline $\begin{array}{l}\text { MAPE } \\
\text { QLIKE }\end{array}$ & $\begin{array}{c}0.5372 \\
2.0652^{* * *}\end{array}$ & $\begin{array}{l}0.5743^{* *} \\
2.0869^{* *}\end{array}$ & $\begin{array}{l}0.5942^{* *} \\
2.0980^{* *}\end{array}$ \\
\hline & & & HAR-CJ \\
\hline c & -0.1526 & -0.1556 & -0.1641 \\
\hline$I^{(1)}$ & $\begin{array}{c}(-0.8698) \\
0.0606\end{array}$ & $\begin{array}{c}(-1.5025) \\
0.0208\end{array}$ & $\begin{array}{c}(-1.8874) \\
0.0223\end{array}$ \\
\hline J11 & (1.8315) & $(1.0752)$ & $(1.2972)$ \\
\hline$J^{(5)}$ & 0.1464 & 0.1650 & 0.14 \\
\hline & $(2.9161)$ & (5.5419) & $(5.4709)$ \\
\hline$J^{(22)}$ & $\begin{array}{l}0.1885 \\
(2.9139)\end{array}$ & $\begin{array}{c}0.1877 \\
(4.9581)\end{array}$ & $\begin{array}{c}0.2042 \\
(6.3064)\end{array}$ \\
\hline$C^{(1)}$ & 0.1160 & 0.0503 & 0.0410 \\
\hline & $(5.4489)$ & $(3.9183)$ & $(3.7223)$ \\
\hline$C^{(5)}$ & $\begin{array}{l}0.1461 \\
(3.4427)\end{array}$ & $\begin{array}{l}0.1623 \\
(6.8289)\end{array}$ & $\begin{array}{c}0.1675 \\
(7.7370)\end{array}$ \\
\hline & 0.2726 & 0.3013 & 0.2914 \\
\hline$C^{(22)}$ & $(5.2465)$ & $(10.1374)$ & $(11.1958)$ \\
\hline adj. $R^{2}$ & 0.4395 & 0.6592 & 0.7060 \\
\hline PE & 2.2207 & $2.3076^{* *}$ & $2.3585^{* *}$ \\
\hline $\begin{array}{l}\text { MAPE } \\
\text { QLIKE }\end{array}$ & $\begin{array}{c}0.5350 \\
2.0642^{* *}\end{array}$ & $\begin{array}{l}0.5753^{* *} \\
2.0884^{* *}\end{array}$ & $\begin{array}{l}0.5947^{* *} \\
2.0980^{* *}\end{array}$ \\
\hline & & & HAR-PS \\
\hline c & 0.178 & 0.1408 & 0.15 \\
\hline & & $(6.75$ & \\
\hline$R S^{+}$ & $\begin{array}{c}0.0559 \\
(2.0366)\end{array}$ & 0.0168 & 0.0151 \\
\hline$B S^{-}$ & 0.08 & 0.04 & 0.03 \\
\hline KS & (2.5813) & (2.1253) & $(2.1929)$ \\
\hline $\mathbf{I}_{r_{t}<0} R V^{(1)}$ & 0.0384 & 0.01 & 0.00 \\
\hline & (1.4279) & $(0.6939)$ & $(0.2324)$ \\
\hline$V^{(5)}$ & $\begin{array}{l}0.2861 \\
(5.0307)\end{array}$ & $\begin{array}{l}0.3202 \\
(9.7252)\end{array}$ & $\begin{array}{l}0.3107 \\
(106517\end{array}$ \\
\hline DY (22) & & $\begin{array}{c}(9.5019) \\
0.5019\end{array}$ & 0.5008 \\
\hline$R V^{(22)}$ & $(8.5650)$ & $(15.4629)$ & $(17.5027)$ \\
\hline adj. $R^{2}$ & 0.439 & 0.6570 & 0.70 \\
\hline T. & 0.459 & 0 & 0.30 \\
\hline & 0.5377 & $0.5742^{* *}$ & $0.5943^{* *}$ \\
\hline LIKE & $2.0649^{* *}$ & $2.0870^{* *}$ & $2.0980^{* *}$ \\
\hline & & & HAR-CJ \\
\hline c & -0.1110 & & -0.0642 \\
\hline & $(-0.6211)$ & $(-0.6377)$ & $(-0.7360)$ \\
\hline$J^{(1)}$ & $\begin{array}{l}0.0500 \\
(1.5437)\end{array}$ & $\begin{array}{c}0.0156 \\
(0.8158)\end{array}$ & $\begin{array}{c}0.0182 \\
(1.0629)\end{array}$ \\
\hline$J^{(5)}$ & 0.1410 & 0.1579 & 0.1333 \\
\hline & (2.8643) & $(5.4661)$ & (5.3211) \\
\hline$J^{(22)}$ & $\begin{array}{r}0.159 \\
(2.418\end{array}$ & $\begin{array}{l}0.14 \\
(3.91\end{array}$ & 0.16 \\
\hline & $(2.41$ & $(3.9$ & \\
\hline$C^{(1)}$ & $\begin{array}{c}0.0984 \\
(4.5401)\end{array}$ & $\begin{array}{c}0.0420 \\
(3.2324)\end{array}$ & $\begin{array}{c}0.0351 \\
(3.1438)\end{array}$ \\
\hline$C^{(}$ & 0.1548 & 0.1647 & 0.1676 \\
\hline$C^{\mathrm{t}}$ & $(3.6572)$ & (6.8945) & $(7.7898)$ \\
\hline 22) & 0.2811 & 0.3173 & $\begin{array}{l}0.3099 \\
\end{array}$ \\
\hline & $(5.4272)$ & $(10.7287)$ & \\
\hline$r_{t}^{-,(1)}$ & $\begin{array}{c}-0.0790 \\
(-4.6098)\end{array}$ & $\begin{array}{r}-0.0228 \\
(-2.1229)\end{array}$ & $\begin{array}{l}-0.0086 \\
(-0.9357)\end{array}$ \\
\hline & -0.0090 & -0.0145 & -0.0154 \\
\hline & $\begin{array}{l}(-1 \\
(-1)\end{array}$ & $(-5.0678)$ & $(-6.0187)$ \\
\hline$r_{t}^{-,(22)}$ & (1) & 81) & \\
\hline adj. $R^{2}$ & & & \\
\hline MSPE & $2.1821^{* *}$ & $2.3030^{* *}$ & $2.3561^{* *}$ \\
\hline MAPE & 0.5278 & $0.5716^{* *}$ & $0.5915^{* *}$ \\
\hline QLIKI & $2.0595^{* *}$ & $2.0852^{* *}$ & $2.0953^{* *}$ \\
\hline
\end{tabular}

Table 8: In-Sample regression results for Rough Rice with $R V$. Note that * and ** indicate the inclusion in the $\mathcal{M}_{90 \%}^{*}$ and $\mathcal{M}_{75 \%}^{*}$, respectively. The $t$-statistics for the parameter estimates are given in parentheses. 


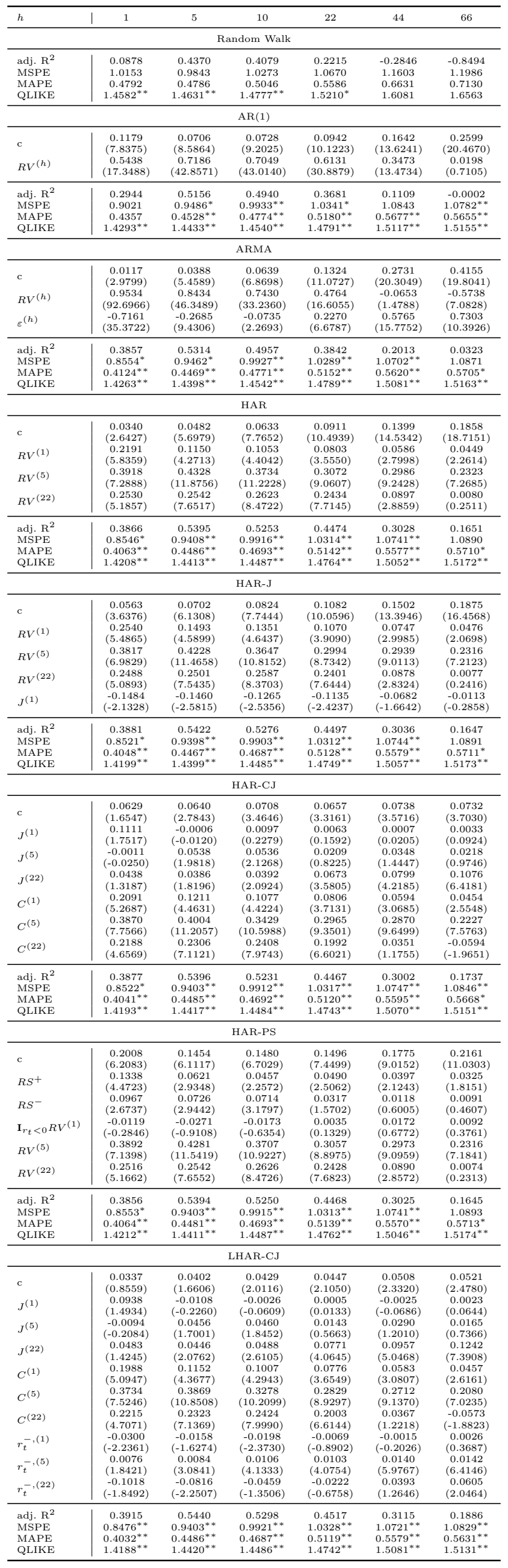

Table 9: In-Sample regression results for Soybean with $R V$. Note that * and ** indicate the inclusion in the $\mathcal{M}_{90 \%}^{*}$ and $\mathcal{M}_{75 \%}^{*}$, respectively. The $t$-statistics for the parameter estimates are given in parentheses. 


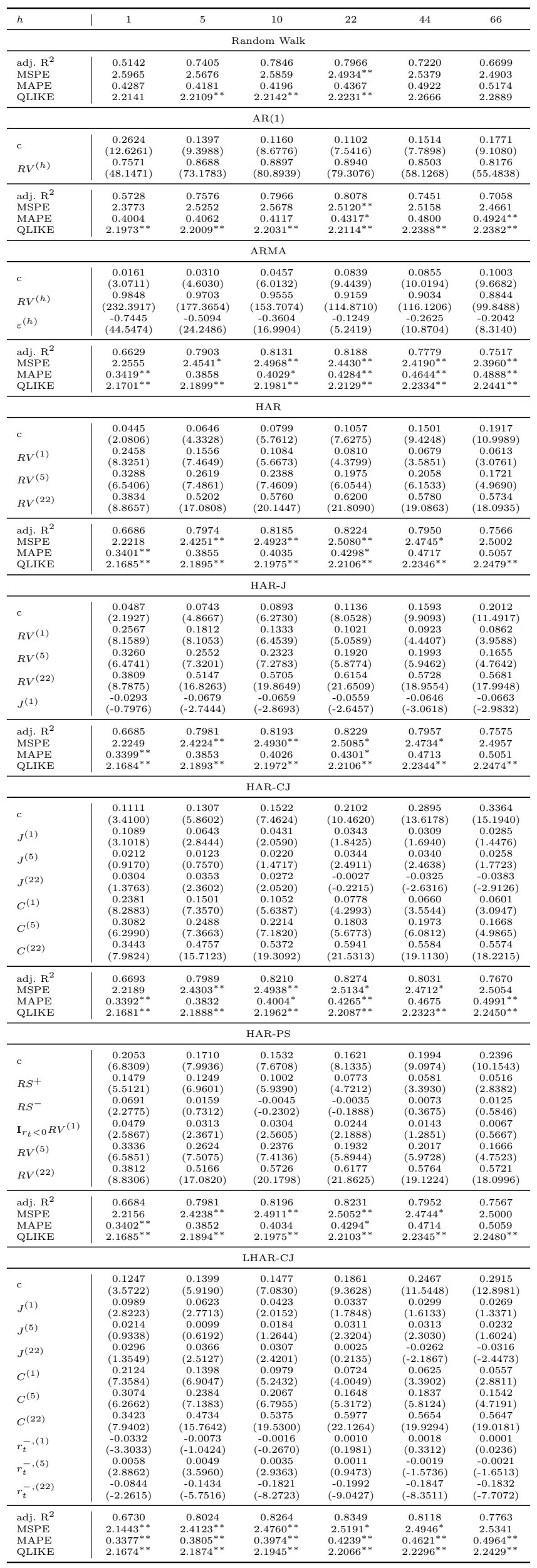

Table 10: In-Sample regression results for Sugar with $R V$. Note that ${ }^{*}$ and ${ }^{* *}$ indicate the inclusion in the $\mathcal{M}_{90 \%}^{*}$ and $\mathcal{M}_{75 \%}^{*}$, respectively. The $t$-statistics for the parameter estimates are given in parentheses. 


\begin{tabular}{|c|c|c|c|c|}
\hline$h$ & 1 & 5 & 10 & 22 \\
\hline & & & ddom Walk & \\
\hline adj. $R^{2}$ & 0.1756 & 0.4970 & 0.5112 & 0.5163 \\
\hline $\begin{array}{l}\text { adj. } \\
\text { MSPE }\end{array}$ & $\begin{array}{l}0.156 \\
2.0653\end{array}$ & $\begin{array}{l}0.4900 \\
2.1759\end{array}$ & $2.1633^{* * *}$ & $\begin{array}{l}0.5163 \\
2.1861\end{array}$ \\
\hline MAPE & 0.4737 & 0.4704 & 0.4849 & 0.4974 \\
\hline QLIKE & 2.1772 & $2.1701^{* *}$ & $2.1787^{* *}$ & $2.1912^{* *}$ \\
\hline & & & $\operatorname{AR}(1)$ & \\
\hline $\begin{array}{l}\text { c } \\
R V^{(h)}\end{array}$ & $\begin{array}{c}0.4086 \\
(12.9311) \\
0.5877 \\
(20.7364)\end{array}$ & $\begin{array}{c}0.2484 \\
(14.4719) \\
0.7482 \\
(45.0175)\end{array}$ & $\begin{array}{c}0.2413 \\
(14.4099) \\
0.7551 \\
(44.6687)\end{array}$ & $\begin{array}{c}0.2344 \\
(14.3526) \\
0.7588 \\
(47.0778)\end{array}$ \\
\hline adj. $\mathrm{R}^{2}$ & 0.3446 & 0.5600 & 0.5708 & 0.5740 \\
\hline MSPE & 1.9101 & 2.0802 & $2.1081^{* *}$ & $2.1652^{* *}$ \\
\hline MAPE & 0.4188 & 0.4432 & 0.4569 & $0.4708^{* * *}$ \\
\hline QLIKE & $2.1400^{* *}$ & $2.1492^{* *}$ & $2.1576^{* *}$ & $2.1650^{* *}$ \\
\hline & & & ARMA & \\
\hline & 0.0386 & 0.0857 & 0.1074 & 0.1901 \\
\hline & $(4.4988)$ & $(7.0134)$ & $(7.5451)$ & $(10.8720)$ \\
\hline$R V^{(h)}$ & $\begin{array}{c}0.9612 \\
(123.1420)\end{array}$ & $\begin{array}{c}0.9126 \\
(836537)\end{array}$ & $\begin{array}{c}0.8899 \\
(69.8266)\end{array}$ & $\begin{array}{c}0.8060 \\
(49.5912)\end{array}$ \\
\hline$(h)$ & $\begin{array}{c}(123.1420) \\
-0.7114\end{array}$ & $\begin{array}{c}(83.6537) \\
-0.4264\end{array}$ & $\begin{array}{l}(69.8266) \\
-0.3405\end{array}$ & $\begin{array}{l}(49.5912) \\
-0.1030\end{array}$ \\
\hline$\varepsilon^{(n)}$ & $(39.6273)$ & (16.9608) & (11.9844) & $(3.4798)$ \\
\hline adj. $R^{2}$ & 0.4496 & 0.5878 & 0.5937 & 0.5820 \\
\hline $\begin{array}{l}\text { MSPE } \\
\text { MAPE }\end{array}$ & $\begin{array}{l}1.8055^{* *} \\
0.384^{* *}\end{array}$ & $\begin{array}{l}2.0460^{*} \\
0.433\end{array}$ & $\begin{array}{l}2.0984^{* *} \\
0.4515\end{array}$ & $\begin{array}{l}2.1601^{* *} \\
0.4704^{* *}\end{array}$ \\
\hline QLIKE & $\begin{array}{l}0.3884 * * \\
2.1248^{* *}\end{array}$ & $2.1451^{* *}$ & $2.1556^{* * *}$ & $2.1650^{* * *}$ \\
\hline & & & HAR & \\
\hline c & $\begin{array}{l}0.0978 \\
(3.5555)\end{array}$ & 0.1410 & 0.1791 & 0.2264 \\
\hline & 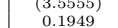 & $\begin{array}{c}(7.6910) \\
0.1535\end{array}$ & $\begin{array}{c}(10.5290) \\
0.1083\end{array}$ & $\begin{array}{l}(14.3942) \\
0.0804\end{array}$ \\
\hline$R V^{(1)}$ & (5.3468) & $\begin{array}{l}(.1 .9368) \\
(5.936)\end{array}$ & $\begin{array}{l}(4.4552) \\
\text { (4) }\end{array}$ & $(3.5647)$ \\
\hline$R V^{(5)}$ & 0.4178 & 0.3043 & 0.2502 & 0.1886 \\
\hline & $\begin{array}{l}(7.9222) \\
0.2885\end{array}$ & $\begin{array}{l}(8.3324) \\
0.3987\end{array}$ & $(7.4342)$ & $(5.8225)$ \\
\hline$R V^{(22)}$ & $\begin{array}{c}0.2885 \\
(5.5246)\end{array}$ & $\begin{array}{c}0.3987 \\
(11.7133)\end{array}$ & $\begin{array}{c}0.4589 \\
(14.9318)\end{array}$ & $\begin{array}{c}0.4985 \\
(18.3043)\end{array}$ \\
\hline adj. $R^{2}$ & 0.4549 & 0.6061 & 0.6090 & 0.6061 \\
\hline $\begin{array}{l}\text { MSPE } \\
\text { MAPE }\end{array}$ & $\begin{array}{l}1.7985^{* *} \\
0.3848^{* * *}\end{array}$ & $\begin{array}{c}2.0307^{* *} \\
0.4272^{*}\end{array}$ & $\begin{array}{c}2.1063^{* *} \\
0.4496\end{array}$ & $\begin{array}{l}2.1733 \\
0.4702^{* *}\end{array}$ \\
\hline QLIKE & $2.1241^{* *}$ & $2.1412^{* *}$ & $2.1534^{* *}$ & $2.1635^{* *}$ \\
\hline & & & HAR-J & \\
\hline c & $\begin{array}{l}0.0917 \\
(3.3578)\end{array}$ & 0.1397 & 0.1797 & 0.2268 \\
\hline & $\begin{array}{c}(3.3578) \\
0.1803\end{array}$ & 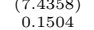 & $\begin{array}{l}\left(\begin{array}{c}(0.3125) \\
0.1097\end{array}\right. \\
0\end{array}$ & 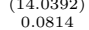 \\
\hline$R V^{(1)}$ & $\begin{array}{l}0.1803 \\
(4.4874)\end{array}$ & $\begin{array}{l}0.1004 \\
(5.2974)\end{array}$ & $(4.1279)$ & $\begin{array}{l}0.0814 \\
(3.2821)\end{array}$ \\
\hline$R V^{(5)}$ & $\begin{array}{l}0.4181 \\
\end{array}$ & 0.3044 & 0.2502 & 0.1885 \\
\hline & $(7.9558)$ & $(8.3380)$ & $(7.4288)$ & $(5.8194)$ \\
\hline$R V^{(22)}$ & $\begin{array}{r}0.2899 \\
(5.5635)\end{array}$ & $\begin{array}{c}0.3990 \\
(11.7119)\end{array}$ & $\begin{array}{c}0.4587 \\
(14.8971)\end{array}$ & $\begin{array}{c}0.4984 \\
(18.2686)\end{array}$ \\
\hline$J^{(1)}$ & $\begin{array}{c}(5.5635) \\
0.0510\end{array}$ & $\begin{array}{c}(11.0119) \\
0.0110\end{array}$ & $\begin{array}{c}(14.8971) \\
-0.0048\end{array}$ & $\begin{array}{c}(18.2686) \\
-0.0035\end{array}$ \\
\hline$J^{(1)}$ & (1.1025) & $(0.3367)$ & $(-0.1597)$ & $(-0.1268)$ \\
\hline adj. $\mathrm{R}^{2}$ & 0.4551 & 0.6059 & 0.60 & 0.60 \\
\hline & $1.7973^{* * *}$ & $2.0314^{*}$ & $2.1064^{* *}$ & 2.1733 \\
\hline & $\begin{array}{l}0.3849^{* *} \\
2.1241^{* *}\end{array}$ & $\begin{array}{c}0.4275^{*} \\
21414 * *\end{array}$ & $\begin{array}{c}0.4497 \\
2.1534^{* *}\end{array}$ & $\begin{array}{l}0.4701^{* *} \\
2.1635^{* *}\end{array}$ \\
\hline & & $2.1414^{*}$ & & 2.1035 \\
\hline & & & HAR-CJ & \\
\hline c & 0.1324 & 0.155 & 0.1654 & 0.1499 \\
\hline (1) & $\begin{array}{l}(2.9277) \\
0.1112\end{array}$ & $\begin{array}{l}(5.0306) \\
0.0860\end{array}$ & $\begin{array}{c}(5.4939) \\
0.0511\end{array}$ & $\begin{array}{c}(5.6053) \\
0.0376\end{array}$ \\
\hline$J^{(1)}$ & $(2.8383)$ & $(3.0474)$ & $\begin{array}{l}0.0311 \\
(1.9466)\end{array}$ & $(1.5871)$ \\
\hline$J^{(5)}$ & $\begin{array}{l}0.0234 \\
(0.8042)\end{array}$ & 0.0007 & 0.0123 & -0.0136 \\
\hline & $\begin{array}{l}(0.8042) \\
0.0522\end{array}$ & $(0.0325)$ & $(0.6412)$ & $(-0.8228)$ \\
\hline$J^{(22)}$ & $\begin{array}{l}0.0522 \\
(1.7119)\end{array}$ & $\begin{array}{c}0.0718 \\
(3.3663)\end{array}$ & $\begin{array}{c}0.0868 \\
(4.2404)\end{array}$ & $\begin{array}{c}0.1371 \\
(77988)\end{array}$ \\
\hline$C^{(1)}$ & 0.1886 & 0.1444 & $\begin{array}{l}(4.24034) \\
0.1034\end{array}$ & 0.0767 \\
\hline$C^{(1)}$ & (5.6975) & $(6.2422)$ & $(4.7888)$ & $(3.8145)$ \\
\hline$C^{(5)}$ & $\begin{array}{l}0.3912 \\
(8.1138\end{array}$ & $\begin{array}{l}0.2976 \\
(8825)\end{array}$ & 0.2427 & 0.1944 \\
\hline & $(8.1138)$ & $(8.8225)$ & $(7.8325)$ & $(6.5556)$ \\
\hline$C^{(22)}$ & $\begin{array}{c}0.2413 \\
(4.8565)\end{array}$ & $\begin{array}{c}0.3340 \\
(9.9894)\end{array}$ & $\begin{array}{c}0.3821 \\
(12.5673)\end{array}$ & $\begin{array}{c}0.3869 \\
(14.5925)\end{array}$ \\
\hline adj. $R^{2}$ & 0.4566 & 0.6095 & 0.6126 & 0.6126 \\
\hline $\begin{array}{l}\text { MSPE } \\
\text { MAPE }\end{array}$ & $\begin{array}{l}1.7950^{* *} \\
0.3835^{* * *}\end{array}$ & $\begin{array}{l}2.0249^{* *} \\
0.4258^{* *}\end{array}$ & $\begin{array}{c}2.1039^{* *} \\
0.4486\end{array}$ & $\begin{array}{l}2.1589^{* *} \\
0.4697^{* *}\end{array}$ \\
\hline QLIKE & $2.1231^{* *}$ & $2.1403^{* *}$ & $2.1529^{* * *}$ & $2.1645^{* *}$ \\
\hline & & & HAR-PS & \\
\hline c & 0.2400 & 0.251 & 0.255 & 0.28 \\
\hline & $\begin{array}{l}(6.4359) \\
0.1301\end{array}$ & $(9.112$ & $(9.8467)$ & $\begin{array}{c}(11.9562) \\
0.0551\end{array}$ \\
\hline$R S^{+}$ & $\begin{array}{l}0.1301 \\
(4.7287)\end{array}$ & $\begin{array}{l}0.0930 \\
(4.9477)\end{array}$ & $\begin{array}{c}0.0711 \\
(4.2080)\end{array}$ & $\begin{array}{l}0.0551 \\
(3.2455)\end{array}$ \\
\hline$R S^{-}$ & 0.0649 & 0.0587 & 0.0330 & 0.0185 \\
\hline & $(1.5032)$ & $(1.7615)$ & (1.0594) & $(0.6888)$ \\
\hline $\mathbf{I}_{r_{t}<0} R V^{(1)}$ & $\begin{array}{c}-0.0265 \\
(-1.0440)\end{array}$ & $\begin{array}{l}-0.0218 \\
(-1.1457)\end{array}$ & $\begin{array}{l}-0.0099 \\
(-0.5667)\end{array}$ & $\begin{array}{c}-0.0008 \\
(-0.0497)\end{array}$ \\
\hline$R V^{(5)}$ & 0.4304 & 0.3168 & 0.2590 & 0.1954 \\
\hline & $(8.0612)$ & $(8.4425)$ & $(7.5256)$ & $(5.9467)$ \\
\hline$R V^{(22)}$ & $\begin{array}{c}0.2901 \\
(5.5336)\end{array}$ & $\begin{array}{c}0.3997 \\
(11.7027)\end{array}$ & $\begin{array}{c}0.4595 \\
(14.9139)\end{array}$ & $\begin{array}{c}0.4987 \\
(18.2757)\end{array}$ \\
\hline adj. $R^{2}$ & 0.4552 & 0.6053 & 0.6085 & 0.6054 \\
\hline MSPE & $1.7932^{* *}$ & $2.0286^{* * *}$ & 2.106 & 2.1 \\
\hline MA & $0.3852^{* *}$ & $0.4260^{* *}$ & 0.4504 & $0.4701^{* *}$ \\
\hline QLIKE & $2.1244^{* *}$ & $2.1403^{* *}$ & $2.1538^{* *}$ & $2.1636^{* *}$ \\
\hline & & & HAR-CJ & \\
\hline c & 0.1186 & 0.1330 & 0.1413 & 0.1257 \\
\hline & $(2.5508)$ & & & \\
\hline$J^{(1)}$ & 0.1122 & 0.0870 & $\begin{array}{c}0.0522 \\
(1.9930)\end{array}$ & $\begin{array}{l}0.0375 \\
01.580\end{array}$ \\
\hline$J^{(5)}$ & 0.0185 & -0.0073 & 0.0042 & $\begin{array}{l}1.0007 \\
-0.0209\end{array}$ \\
\hline$J^{(5)}$ & $(0.6362)$ & $(-0.3484)$ & $(0.2179)$ & $(-1.2763)$ \\
\hline$J^{(}$ & 0.0518 & 0.0721 & 0.0863 & 0.1364 \\
\hline & (1.7068) & $(3.3859)$ & $(4.2261)$ & $(7.8382)$ \\
\hline$C^{(1}$ & 0.1885 & 0.1439 & 0.10 & 0.0755 \\
\hline & & (6.2717) & (4.8431) & $(3.8$ \\
\hline$C^{(5)}$ & 0.3851 & 0.2878 & 0.2328 & 0.1853 \\
\hline & $(8.0231)$ & $(8.5897)$ & $(7.6340)$ & $(6.3103)$ \\
\hline$C^{(22)}$ & $\begin{array}{l}0.2527 \\
(5.1051)\end{array}$ & $\begin{array}{c}0.3504 \\
(10.5295)\end{array}$ & $\begin{array}{c}0.4009 \\
(13.2915)\end{array}$ & $\begin{array}{c}0.4051 \\
(15.3738)\end{array}$ \\
\hline$r-r$ & 0.0026 & 0.0017 & 0.0027 & -0.0004 \\
\hline$r_{t}$ & $(0.2422)$ & $(0.2386)$ & $(0.4048)$ & $(-0.0588)$ \\
\hline$r_{t}^{-,(5)}$ & $\begin{array}{c}-0.0007 \\
(-0.2089\end{array}$ & $\begin{array}{r}-0.0003 \\
-(-0.1225\end{array}$ & $\begin{array}{r}-0.0016 \\
-(-08084\end{array}$ & $\begin{array}{l}-0.0024 \\
-(-1.22255\end{array}$ \\
\hline & -0.1093 & $\begin{array}{c}(-0.1225) \\
-0.1662\end{array}$ & $\begin{array}{c}(-0.8084) \\
-0.1799\end{array}$ & $\begin{array}{l}(-1.2225) \\
-0.1700\end{array}$ \\
\hline$r_{t}^{-,(22)}$ & $(-1.9617)$ & $(-4.8605)$ & $(-6.0103)$ & $(-6.3813)$ \\
\hline adj. $\mathrm{R}^{2}$ & & 143 & & \\
\hline MSPE & $1.7909^{* *}$ & $2.0198^{* *}$ & $2.1030^{* * *}$ & $\begin{array}{l}2.1556^{* *} \\
0.465^{* *}\end{array}$ \\
\hline $\begin{array}{l}\text { MAPE } \\
\text { QLIKE }\end{array}$ & $\begin{array}{l}0.38374^{* *} \\
2.123\end{array}$ & $\begin{array}{l}0.42337^{* *} \\
2.1399^{* *}\end{array}$ & $\begin{array}{l}0.44472^{* *} \\
2.152^{2}\end{array}$ & $\begin{array}{l}0.4665^{* *} \\
2.1633^{* *}\end{array}$ \\
\hline
\end{tabular}

Table 11: In-Sample regression results for Wheat with $R V$. Note that * and ** indicate the inclusion in the $\mathcal{M}_{90 \%}^{*}$ and $\mathcal{M}_{75 \%}^{*}$, respectively. The $t$-statistics for the parameter estimates are given in parentheses. 
sistently outperformed by any of its extended versions, namely the HAR-J, HAR-CJ, HAR-PS and LHAR-CJ models; a finding that holds for both volatility measures. Hence, building forecasting models based on the jump component, the continuous component, the signed jumps, and the volatility or return leverage does not improve the forecasting accuracy. Thus, there is no scope to add complexity in the predictive models without obtaining a significant predictive gain. This finding is in some contrast to Tian et al. (2017a), Tian et al. (2017b), Yang et al. (2017), and Luo et al. (2019) who maintain that the jump component as well as the introduction of time-varying HAR coefficients help improving forecast accuracy of agricultural commodities price volatility. These results are not contradicting, however. The substantial findings of Tian et al. (2017a), Tian et al. (2017b) and Yang et al. (2017) are derived from data of Chinese futures markets, while our price data comes from North-American markets. Comparing realized volatility measures of these two markets as well as the results of the aforementioned studies shows that Chinese and U.S. futures markets behave differently and are possibly driven by dissimilar factors. One of the most important reasons for this apparent difference of market behavior is the investor's structure in the two markets, where the Chinese market is much more driven by speculators than the U.S. market (Bloomberg, 2019, Fan \& Zhang, 2018, Klein \& Todorova, 2018).

We maintain, though, that the expected outcome for the jump components is not to provide any forecasting gains given that they are built in order to capture the surprised (sudden) changes in volatility. Hence, the jump component is not encompassing any element of either long or short memory; they are simply non-autocorrelated zero mean stochastic processes. This is the reason behind the fact that in the in-sample analysis the jump component is able to provide better fitting of the predictive regressions, whereas this ability no longer exists in a real out-of-sample exercise. Even more, the inability of the leverage effects, of either returns or volatility, to generate significant out-of-sample predictive gains, relatively to the simple HAR model, could be explained by the fact that they are not statistically powerful enough to provide incremental predictive information relative to the heterogeneous beliefs of investors. Another possible explanation might be that previous studies which provide evidence in favour of the predictive ability of the jump components in an out-of-sample setting is due to the incorporation of jump values that are not available to the forecasters at the time that the forecasts are generated, i.e. such studies do not produce real out-of-sample forecasts.

Next, we look at the success ratios of our competing models, as shown at the bottom of Tables [2-[6]. It is rather interesting that we cannot argue that there is a single model which is able to provide a superior directional accuracy. On the contrary, we reach the conclusion that even though the HAR-type models correctly predict the direction of the volatility at a high level (up to $75 \%$ depending on the volatility measure and commodity), these are marginally higher (lower) compared to the Random Walk and AR models for the shorter (longer) forecasting horizons. In any case, however, any differences noticed in 
predicting the future direction of volatility are not statistically significant.

Overall, our findings are in line with Sévi (2014) who, despite the fact that he focuses on an energy commodity volatility (i.e. oil price volatility), reaches the same conclusion as our study; namely that even though sophisticated HAR-type models outperform the simple HAR in an in-sample setting, they are not capable of outperforming it in an outof-sample exercise over different forecasting horizons.

\begin{tabular}{|c|c|c|c|c|c|c|c|c|c|}
\hline & $h$ & RW & AR & ARMA & HAR & HAR-J & HAR-CJ & HAR-PS & LHAR-CJ \\
\hline \multirow{6}{*}{ MSPE } & 1 & 1.3038 & 1.1508 & 1.2400 & $1.0898^{* *}$ & $1.0901^{* *}$ & $1.0890^{* *}$ & 1.0992 & $1.0993^{* *}$ \\
\hline & 5 & $1.2454^{* *}$ & $1.2166^{* *}$ & 1.2963 & $1.2045^{* *}$ & $1.2036^{* *}$ & $1.2031^{* *}$ & $1.2079^{* *}$ & $1.2035^{* *}$ \\
\hline & 10 & 1.2983 & $1.2651^{* *}$ & 1.3346 & 1.2742 & 1.2726 & $1.2626^{* *}$ & 1.2806 & $1.2636^{* *}$ \\
\hline & 22 & 1.3949 & 1.3423 & 1.3875 & $1.3248^{* *}$ & $1.3241^{* *}$ & $1.3197^{* *}$ & $1.3240^{* *}$ & 1.3282 \\
\hline & 44 & 1.4981 & 1.3859 & $1.3376^{* *}$ & $1.3747^{*}$ & $1.3746^{*}$ & $1.3667^{*}$ & $1.3739^{*}$ & $1.3768^{*}$ \\
\hline & 66 & 1.5565 & 1.3870 & 1.4430 & 1.3753 & 1.3759 & $1.3665^{* *}$ & 1.3753 & 1.3760 \\
\hline \multirow{6}{*}{ MAPE } & 1 & 0.6093 & 0.5899 & 0.5988 & $0.5516^{* *}$ & $0.5515^{* *}$ & $0.5509^{* *}$ & 0.5550 & $0.5570^{*}$ \\
\hline & 5 & 0.6232 & 0.6181 & 0.6389 & $0.6056^{* *}$ & $0.6057^{* *}$ & $0.6043^{* *}$ & $0.6070^{* *}$ & $0.6038^{* *}$ \\
\hline & 10 & 0.6325 & $0.6268^{* *}$ & 0.6456 & $0.6282^{*}$ & 0.6288 & $0.6232^{* *}$ & 0.6305 & $0.6240^{* *}$ \\
\hline & 22 & 0.6644 & 0.6593 & 0.6875 & $0.6505^{* *}$ & $0.6506^{* *}$ & $0.6483^{* *}$ & $0.6503^{* *}$ & 0.6541 \\
\hline & 44 & 0.7217 & $0.7002^{* *}$ & $0.7020^{* *}$ & $0.6913^{* *}$ & $0.6917^{* *}$ & $0.6939^{* *}$ & $0.6912^{* *}$ & 0.7016 \\
\hline & 66 & 0.7647 & 0.7313 & 0.7701 & $0.7031^{* *}$ & $0.7039^{*}$ & $0.7094^{*}$ & $0.7032^{* *}$ & 0.7155 \\
\hline \multirow{6}{*}{ QLIKE } & 1 & 1.6887 & 1.6600 & 1.6798 & $1.6517^{* *}$ & $1.6519^{* *}$ & $1.6520^{* *}$ & 1.6535 & $1.6529^{* *}$ \\
\hline & 5 & 1.6925 & $1.6801^{* *}$ & 1.7075 & $1.6770^{* *}$ & $1.6768^{* *}$ & $1.6771^{* *}$ & $1.6770^{* *}$ & $1.6775^{* *}$ \\
\hline & 10 & 1.7014 & $1.6906^{* *}$ & 1.7123 & 1.6959 & $1.6955^{*}$ & $1.6933^{* *}$ & 1.6968 & $1.6940^{* *}$ \\
\hline & 22 & 1.7340 & $1.7165^{* *}$ & 1.7321 & $1.7113^{* *}$ & $1.7113^{* *}$ & $1.7111^{* *}$ & $1.7114^{* *}$ & $1.7139^{* *}$ \\
\hline & 44 & 1.7852 & 1.7320 & $1.7076^{* *}$ & 1.7280 & 1.7278 & 1.7248 & 1.7278 & 1.7287 \\
\hline & 66 & 1.8200 & $1.7205^{* *}$ & 1.7371 & 1.7267 & 1.7267 & $1.7223^{* *}$ & 1.7267 & 1.7265 \\
\hline \multirow{6}{*}{ SR } & 1 & $0.7606^{* * *}$ & $0.7389^{* * *}$ & $0.7597^{* * *}$ & $0.7597^{* * *}$ & $0.7578^{* * *}$ & $0.7635^{* * *}$ & $0.7540^{* * *}$ & $0.7588^{* * *}$ \\
\hline & 5 & $0.6821^{* * *}$ & $0.6689^{* * *}$ & $0.6717^{* * *}$ & $0.6821^{* * *}$ & $0.6802^{* * *}$ & $0.6783^{* * *}$ & $0.6850^{* * *}$ & $0.6840^{* * *}$ \\
\hline & 10 & $0.6774^{* * *}$ & $0.6689^{* * *}$ & $0.6471^{* * *}$ & $0.6481^{* * *}$ & $0.6414^{* * *}$ & $0.6462^{* * *}$ & $0.6518^{* * *}$ & $0.6462^{* * *}$ \\
\hline & 22 & $0.6083^{* * *}$ & $0.5885^{* * *}$ & 0.5260 & $0.6140^{* * *}$ & $0.6140^{* * *}$ & $0.6140^{* * *}$ & $0.6121^{* * *}$ & $0.6159^{* * *}$ \\
\hline & 44 & 0.4787 & 0.4437 & $0.5809^{* * *}$ & $0.5383^{* *}$ & $0.5383^{* *}$ & $0.5393^{* *}$ & $0.5459^{* *}$ & 0.5061 \\
\hline & 66 & 0.4172 & 0.5676 & 0.5118 & 0.5307 & 0.5260 & $0.5506^{* *}$ & 0.5203 & 0.5336 \\
\hline
\end{tabular}

Table 12: Forecasting Evaluation for Corn Futures with $R V$. Note that ${ }^{*}$ and $* *$ indicate the inclusion in the $\mathcal{M}_{90 \%}^{*}$ and $\mathcal{M}_{75 \%}^{*}$, respectively. For the Success Ratio (SR), the asterisk ${ }^{*},{ }^{* *}$, and ${ }^{* * *}$ indicate a statistical significance at $10 \%, 5 \%$, and $1 \%$, respectively.

\begin{tabular}{|c|c|c|c|c|c|c|c|c|c|}
\hline & $h$ & RW & $\mathrm{AR}$ & ARMA & HAR & HAR-J & HAR-CJ & HAR-PS & LHAR-CJ \\
\hline \multirow{6}{*}{ MSPE } & 1 & 2.4100 & 2.1404 & 2.3635 & $1.9934^{* *}$ & $1.9968^{* *}$ & $1.9967^{* *}$ & $1.9943^{* *}$ & $1.9838^{* *}$ \\
\hline & 5 & $2.1062^{* *}$ & $2.1123^{*}$ & $2.1350^{*}$ & $2.0759^{* *}$ & $2.0767^{* *}$ & $2.0843^{* *}$ & $2.0789^{* *}$ & $2.0708^{* *}$ \\
\hline & 10 & $2.1401^{* *}$ & 2.1503 & 2.1769 & $2.1142^{* *}$ & $2.1144^{* *}$ & $2.1258^{* *}$ & $2.1165^{* *}$ & $2.1047^{* *}$ \\
\hline & 22 & $2.1149^{* *}$ & $2.1435^{*}$ & $2.1211^{* *}$ & $2.1460^{*}$ & $2.1472^{*}$ & 2.1589 & $2.1460^{*}$ & $2.1264^{* *}$ \\
\hline & 44 & $2.1017^{* *}$ & 2.1329 & 2.2183 & 2.1556 & 2.1575 & 2.1749 & 2.1570 & 2.1632 \\
\hline & 66 & $2.1603^{* *}$ & 2.2203 & 2.3428 & 2.2066 & 2.2043 & 2.2287 & 2.2063 & 2.2106 \\
\hline \multirow{6}{*}{ MAPE } & 1 & 0.8550 & 0.7907 & 0.8478 & $0.7343^{* *}$ & $0.7327^{* *}$ & $0.7354^{* *}$ & $0.7359^{*}$ & $0.7355^{* *}$ \\
\hline & 5 & 0.7980 & 0.7789 & 0.7986 & $0.7624^{*}$ & $0.7619^{* *}$ & $0.7656^{*}$ & $0.7610^{* *}$ & 0.7707 \\
\hline & 10 & 0.7905 & $0.7788^{* *}$ & 0.8049 & $0.7740^{* *}$ & $0.7734^{* *}$ & 0.7815 & $0.7746^{* *}$ & 0.7920 \\
\hline & 22 & $0.7904^{* *}$ & $0.7902^{* *}$ & $0.7856^{* *}$ & $0.7918^{* *}$ & $0.7927^{* *}$ & $0.7990^{*}$ & $0.7911^{* *}$ & 0.8078 \\
\hline & 44 & $0.7840^{* *}$ & $0.7822^{* *}$ & $0.8042^{*}$ & $0.7945^{* *}$ & $0.7949^{* *}$ & 0.7991* & $0.7945^{* *}$ & 0.8134 \\
\hline & 66 & $0.8042^{* *}$ & $0.8036^{* *}$ & 0.8405 & $0.8082^{* *}$ & $0.8074^{* *}$ & $0.8127^{* *}$ & $0.8086^{* *}$ & 0.8305 \\
\hline \multirow{6}{*}{ QLIKE } & 1 & 2.0261 & 1.9471 & 2.0171 & $1.8804^{* *}$ & $1.8811^{* *}$ & $1.8829^{* *}$ & $1.8807^{* *}$ & $1.8781^{* *}$ \\
\hline & 5 & 1.9278 & 1.9234 & 1.9488 & $1.9089^{* *}$ & $1.9096^{* *}$ & $1.9130^{* *}$ & $1.9096^{* *}$ & $1.9076^{* *}$ \\
\hline & 10 & 1.9465 & 1.9422 & 1.9660 & $1.9264^{* *}$ & $1.9275^{* *}$ & $1.9299^{*}$ & $1.9268^{* *}$ & $1.9185^{* *}$ \\
\hline & 22 & $1.9366^{* *}$ & $1.9386^{* *}$ & $1.9488^{*}$ & $1.9402^{* *}$ & $1.9416^{*}$ & $1.9463^{*}$ & $1.9402^{* *}$ & $1.9310^{* *}$ \\
\hline & 44 & $1.9414^{* *}$ & 1.9537 & 2.0278 & $1.9522^{* *}$ & 1.9540 & 1.9576 & 1.9526 & $1.9440^{* *}$ \\
\hline & 66 & $1.9851^{*}$ & 2.0320 & 2.1097 & $1.9874^{*}$ & $1.9869^{*}$ & 1.9947 & $1.9872^{*}$ & $1.9643^{* *}$ \\
\hline \multirow{6}{*}{ SR } & 1 & $0.7011^{* * *}$ & $0.6556^{* * *}$ & $0.7030^{* * *}$ & $0.7353^{* * *}$ & $0.7268^{* * *}$ & $0.7353^{* * *}$ & $0.7315^{* * *}$ & $0.7334^{* * *}$ \\
\hline & 5 & $0.7078^{* * *}$ & $0.6954^{* * *}$ & $0.7011^{* * *}$ & $0.7097^{* * *}$ & $0.7087^{* * *}$ & $0.7144^{* * *}$ & $0.7106^{* * *}$ & $0.7087^{* * *}$ \\
\hline & 10 & $0.7144^{* * *}$ & $0.6917^{* * *}$ & $0.6869^{* * *}$ & $0.7011^{* * *}$ & $0.7049^{* * *}$ & $0.6973^{* * *}$ & $0.7097^{* * *}$ & $0.7030^{* * *}$ \\
\hline & 22 & $0.7049^{* * *}$ & $0.6860^{* * *}$ & $0.6926^{* * *}$ & $0.6765^{* * *}$ & $0.6727^{* * *}$ & $0.6717^{* * *}$ & $0.6755^{* * *}$ & $0.6992^{* * *}$ \\
\hline & 44 & $0.7021^{* * *}$ & $0.6954^{* * *}$ & $0.6945^{* * *}$ & $0.6917^{* * *}$ & $0.6879^{* * *}$ & $0.6831^{* * *}$ & $0.6926^{* * *}$ & $0.6907^{* * *}$ \\
\hline & 66 & $0.6879^{* * *}$ & $0.6898^{* * *}$ & $0.6651^{* * *}$ & $0.6888^{* * *}$ & $0.6907^{* * *}$ & $0.6708^{* * *}$ & $0.6850^{* * *}$ & $0.6850^{* * *}$ \\
\hline
\end{tabular}

Table 13: Forecasting Evaluation for Rice Futures with $R V$. Note that ${ }^{*}$ and ${ }^{* *}$ indicate the inclusion in the $\mathcal{M}_{90 \%}^{*}$ and $\mathcal{M}_{75 \%}^{*}$, respectively. For the Success Ratio (SR), the asterisk ${ }^{*},{ }^{* *}$, and ${ }^{* * *}$ indicate a statistical significance at $10 \%, 5 \%$, and $1 \%$, respectively. 


\begin{tabular}{|c|c|c|c|c|c|c|c|c|c|}
\hline & $h$ & RW & $\mathrm{AR}$ & ARMA & HAR & HAR-J & HAR-CJ & HAR-PS & LHAR-CJ \\
\hline \multirow{6}{*}{ MSPE } & 1 & 0.9453 & 0.8394 & 0.9104 & $0.7848^{* *}$ & $0.7833^{* *}$ & $0.7841^{* *}$ & $0.7857^{* *}$ & $0.7843^{* *}$ \\
\hline & 5 & $0.8677^{*}$ & $0.8529^{*}$ & 0.8989 & $0.8493^{*}$ & $0.8452^{* *}$ & $0.8475^{*}$ & $0.8495^{*}$ & $0.8525^{*}$ \\
\hline & 10 & 0.9314 & $0.9085^{*}$ & 0.9421 & $0.9041^{*}$ & $0.9015^{* *}$ & 0.9076 & $0.9041^{*}$ & 0.9144 \\
\hline & 22 & 1.0220 & 0.9864 & 1.0355 & $0.9603^{* *}$ & $0.9603^{* *}$ & $0.9647^{*}$ & $0.9609^{* *}$ & 0.9762 \\
\hline & 44 & 1.1335 & $1.0377^{*}$ & $1.0193^{* *}$ & $1.0207^{* *}$ & $1.0216^{* *}$ & $1.0256^{*}$ & $1.0208^{* *}$ & 1.0336 \\
\hline & 66 & 1.1765 & $1.0235^{* *}$ & $1.0325^{* *}$ & $1.0361^{* *}$ & $1.0366^{* *}$ & $1.0329^{* *}$ & $1.0362^{* *}$ & 1.0444 \\
\hline \multirow{6}{*}{ MAPE } & 1 & 0.6157 & 0.6015 & 0.6076 & $0.5549^{*}$ & $0.5531^{* *}$ & $0.5554^{*}$ & $0.5550^{*}$ & $0.5581^{*}$ \\
\hline & 5 & 0.6005 & 0.6005 & 0.6202 & $0.5958^{*}$ & $0.5942^{* *}$ & $0.5968^{*}$ & 0.5968 & 0.6013 \\
\hline & 10 & $0.6296^{* *}$ & $0.6282^{* *}$ & 0.6620 & $0.6261^{* *}$ & $0.6246^{* *}$ & $0.6284^{*}$ & $0.6265^{* *}$ & $0.6295^{*}$ \\
\hline & 22 & 0.6953 & 0.6875 & 0.7305 & $0.6735^{* *}$ & $0.6733^{* *}$ & 0.6766 & 0.6750 & 0.6826 \\
\hline & 44 & 0.7691 & 0.7408 & 0.7513 & $0.7234^{* *}$ & $0.7236^{* *}$ & 0.7279 & $0.7237^{* *}$ & 0.7345 \\
\hline & 66 & 0.8075 & 0.7539 & 0.7613 & $0.7401^{* *}$ & $0.7405^{*}$ & $0.7449^{*}$ & $0.7403^{* *}$ & 0.7517 \\
\hline \multirow{6}{*}{ QLIKE } & 1 & 1.3287 & 1.2983 & 1.3209 & $1.2841^{* *}$ & $1.2837^{* *}$ & $1.2834^{* *}$ & $1.2839^{* *}$ & $1.2834^{* *}$ \\
\hline & 5 & 1.3113 & $1.3026^{*}$ & 1.3277 & $1.3021^{*}$ & $1.3010^{* *}$ & $1.3015^{* *}$ & $1.3020^{*}$ & $1.3029^{*}$ \\
\hline & 10 & 1.3405 & $1.3250^{* *}$ & 1.3462 & $1.3274^{* *}$ & $1.3272^{* *}$ & $1.3288^{*}$ & $1.3275^{* *}$ & 1.3315 \\
\hline & 22 & 1.3985 & 1.3642 & 1.3864 & $1.3500^{* *}$ & $1.3501^{* *}$ & $1.3506^{* *}$ & $1.3501^{* *}$ & 1.3562 \\
\hline & 44 & 1.4695 & 1.3865 & $1.3714^{* *}$ & $1.3786^{* *}$ & $1.3790^{* *}$ & $1.3800^{* *}$ & $1.3786^{* *}$ & 1.3853 \\
\hline & 66 & 1.5064 & $1.3769^{* *}$ & $1.3833^{* *}$ & $1.3850^{* *}$ & $1.3852^{* *}$ & $1.3805^{* *}$ & $1.3851^{* *}$ & 1.3885 \\
\hline \multirow{6}{*}{ SR } & 1 & $0.7787^{* * *}$ & $0.7495^{* * *}$ & $0.7768^{* * *}$ & $0.7910^{* * *}$ & $0.7900^{* * *}$ & $0.7834^{* * *}$ & $0.7863^{* * *}$ & $0.7900^{* * *}$ \\
\hline & 5 & $0.7589^{* * *}$ & $0.7580^{* * *}$ & $0.7354^{* * *}$ & $0.7561^{* * *}$ & $0.7571^{* * *}$ & $0.7589^{* * *}$ & $0.7524^{* * *}$ & $0.7571^{* * *}$ \\
\hline & 10 & $0.7288^{* * *}$ & $0.7147^{* * *}$ & $0.6610^{* * *}$ & $0.7175^{* * *}$ & $0.7194^{* * *}$ & $0.7109^{* * *}$ & $0.7175^{* * *}$ & $0.7015^{* * *}$ \\
\hline & 22 & $0.6252^{* * *}$ & $0.5979^{* * *}$ & $0.5490^{*}$ & $0.6412^{* * *}$ & $0.6450^{* * *}$ & $0.6525^{* * *}$ & $0.6431^{* * *}$ & $0.6478^{* * *}$ \\
\hline & 44 & 0.5160 & 0.4595 & $0.5414^{* *}$ & 0.5301 & 0.5264 & 0.5339 & 0.5273 & 0.5188 \\
\hline & 66 & 0.4030 & 0.5075 & 0.4812 & 0.4699 & 0.4614 & 0.4859 & 0.4557 & 0.4492 \\
\hline
\end{tabular}

Table 14: Forecasting Evaluation for Soy Futures with $R V$. Note that ${ }^{*}$ and ${ }^{* *}$ indicate the inclusion in the $\mathcal{M}_{90 \%}^{*}$ and $\mathcal{M}_{75 \%}^{*}$, respectively. For the Success Ratio (SR), the asterisk *, **, and *** indicate a statistical significance at $10 \%, 5 \%$, and $1 \%$, respectively.

\subsection{Real out-of-sample forecasting results: Further tests}

In this section we proceed with the evaluation of the average forecasts over the different horizons, against the actual average volatility over the same horizon. This comparison is motivated by the fact that a number of different stakeholders who are interested in agricultural commodity volatility forecasting (agricultural firms, policy makers, international institutions, etc.) do not require point forecasts at a particular $h$-day ahead horizon, but rather with the expected average volatility over an $h$-period ahead. For brevity, we do not include the results in the main part of the study, but make them available in Appendix B.

Overall, the results based on the MCS test suggest that our main conclusions still hold, providing further evidence that the HAR extensions are not capable of generating any incremental predictive gains, relatively to the HAR model U.S. agricultural commodity markets. This holds for both $R V$ and $M e d R V$ volatility measures. Even more, we report that as we move further out the forecasting horizons, the RW is also included in the set of the best forecasting models, particularly for the $M e d R V$. This is suggestive of the fact that the ability of the HAR model to generate superior average volatility forecasts is economically valuable primarily in shorter horizons. 


\begin{tabular}{|c|c|c|c|c|c|c|c|c|c|}
\hline & $h$ & RW & $\mathrm{AR}$ & ARMA & HAR & HAR-J & HAR-CJ & HAR-PS & LHAR-CJ \\
\hline \multirow{6}{*}{ MSPE } & 1 & 1.6238 & 1.4626 & 1.5919 & $1.3174^{* *}$ & $1.3168^{* *}$ & $1.3167^{* *}$ & $1.3188^{* *}$ & $1.3190^{* *}$ \\
\hline & 5 & 1.4880 & 1.4721 & 1.4925 & $1.4265^{* *}$ & $1.4223^{* *}$ & $1.4225^{* *}$ & $1.4258^{* *}$ & $1.4279^{* *}$ \\
\hline & 10 & $1.4526^{* *}$ & $1.4590^{* *}$ & 1.5383 & $1.4582^{* *}$ & $1.4564^{* *}$ & $1.4519^{* *}$ & $1.4571^{* *}$ & $1.4528^{* *}$ \\
\hline & 22 & $1.5126^{* *}$ & $1.5350^{* *}$ & 1.5995 & $1.5378^{* *}$ & $1.5349^{* *}$ & $1.5329^{* *}$ & $1.5373^{* *}$ & $1.5356^{* *}$ \\
\hline & 44 & $1.5704^{* *}$ & 1.6529 & $1.6063^{*}$ & 1.6275 & 1.6225 & 1.6265 & 1.6281 & 1.6441 \\
\hline & 66 & $1.5623^{* *}$ & 1.6836 & 1.6753 & 1.7081 & 1.7020 & 1.6944 & 1.7084 & 1.6723 \\
\hline \multirow{6}{*}{ MAPE } & 1 & 0.6537 & 0.6265 & 0.6480 & $0.5741^{* *}$ & $0.5748^{* *}$ & $0.5763^{*}$ & $0.5744^{* *}$ & $0.5784^{*}$ \\
\hline & 5 & 0.6427 & 0.6292 & 0.6403 & $0.6119^{* *}$ & $0.6122^{* *}$ & $0.6145^{* *}$ & $0.6114^{* *}$ & $0.6133^{* *}$ \\
\hline & 10 & $0.6358^{*}$ & $0.6304^{* *}$ & 0.6515 & $0.6224^{* *}$ & $0.6225^{* *}$ & $0.6246^{* *}$ & $0.6224^{* *}$ & $0.6241^{* *}$ \\
\hline & 22 & 0.6603 & $0.6468^{* *}$ & $0.6723^{*}$ & $0.6431^{* *}$ & $0.6442^{* *}$ & $0.6507^{*}$ & $0.6433^{* *}$ & $0.6503^{* *}$ \\
\hline & 44 & 0.6878 & $0.6651^{* *}$ & $0.6898^{*}$ & $0.6721^{* *}$ & $0.6728^{* *}$ & 0.6843 & $0.6724^{* *}$ & $0.6846^{*}$ \\
\hline & 66 & 0.6836 & $0.6659^{* *}$ & 0.7222 & 0.6871 & 0.6872 & 0.6949 & 0.6876 & 0.6891 \\
\hline \multirow{6}{*}{ QLIKE } & 1 & 1.9622 & 1.9459 & 1.9591 & $1.9182^{* *}$ & $1.9179^{* *}$ & $1.9177^{* *}$ & $1.9189^{* *}$ & $1.9180^{* *}$ \\
\hline & 5 & 1.9610 & 1.9542 & 1.9604 & $1.9458^{* *}$ & $1.9452^{* *}$ & $1.9439^{* *}$ & $1.9452^{* *}$ & $1.9446^{* *}$ \\
\hline & 10 & $1.9529^{* *}$ & $1.9508^{* *}$ & 1.9833 & $1.9541^{* *}$ & $1.9537^{* *}$ & $1.9510^{* *}$ & $1.9542^{* *}$ & $1.9516^{* *}$ \\
\hline & 22 & $1.9824^{* *}$ & $1.9879^{*}$ & 2.0484 & $1.9854^{*}$ & $1.9843^{*}$ & $1.9791^{* *}$ & $1.9851^{*}$ & $1.9781^{* *}$ \\
\hline & 44 & $2.0311^{* *}$ & 2.0622 & $2.0307^{* *}$ & 2.0484 & 2.0461 & $2.0352^{* *}$ & 2.0490 & $2.0366^{* *}$ \\
\hline & 66 & $2.0122^{* *}$ & 2.0659 & 2.0453 & 2.0752 & 2.0732 & 2.0587 & 2.0762 & 2.0551 \\
\hline \multirow{6}{*}{ SR } & 1 & $0.7333^{* * *}$ & $0.7352^{* * *}$ & $0.7362^{* * *}$ & $0.7662^{* * *}$ & $0.7624^{* * *}$ & $0.7681^{* * *}$ & $0.7624^{* * *}$ & $0.7718^{* * *}$ \\
\hline & 5 & $0.7352^{* * *}$ & $0.7277^{* * *}$ & $0.7211^{* * *}$ & $0.7343^{* * *}$ & $0.7352^{* * *}$ & $0.7390 * * *$ & $0.7399^{* * *}$ & $0.7390^{* * *}$ \\
\hline & 10 & $0.7230^{* * *}$ & $0.7362^{* * *}$ & $0.7052^{* * *}$ & $0.7239^{* * *}$ & $0.7324^{* * *}$ & $0.7174^{* * *}$ & $0.7192^{* * *}$ & $0.7230^{* * *}$ \\
\hline & 22 & $0.7108^{* * *}$ & $0.7146^{* * *}$ & $0.6854^{* * *}$ & $0.7221^{* * *}$ & $0.7080^{* * *}$ & $0.6977^{* * *}$ & $0.7239^{* * *}$ & $0.7136^{* * *}$ \\
\hline & 44 & $0.7174^{* * *}$ & $0.7042^{* * *}$ & $0.7202^{* * *}$ & $0.6920^{* * *}$ & $0.6854^{* * *}$ & $0.6845^{* * *}$ & $0.6901^{* * *}$ & $0.6845^{* * *}$ \\
\hline & 66 & $0.7484^{* * *}$ & $0.6723^{* * *}$ & $0.6704^{* * *}$ & $0.6563^{* * *}$ & $0.6516^{* * *}$ & $0.6638^{* * *}$ & $0.6516^{* * *}$ & $0.6714^{* * *}$ \\
\hline
\end{tabular}

Table 15: Forecasting Evaluation for Sugar Futures with $R V$. Note that ${ }^{*}$ and ${ }^{* *}$ indicate the inclusion in the $\mathcal{M}_{90 \%}^{*}$ and $\mathcal{M}_{75 \%}^{*}$, respectively. For the Success Ratio (SR), the asterisk *, **, and *** indicate a statistical significance at $10 \%, 5 \%$, and $1 \%$, respectively.

\begin{tabular}{|c|c|c|c|c|c|c|c|c|c|}
\hline & $h$ & RW & $\mathrm{AR}$ & ARMA & HAR & HAR-J & HAR-CJ & HAR-PS & LHAR-CJ \\
\hline \multirow{6}{*}{ MSPE } & 1 & 1.4871 & 1.3285 & 1.4444 & $1.2476^{* *}$ & $1.2497^{* *}$ & $1.2575^{*}$ & $1.2418^{* *}$ & $1.2579^{*}$ \\
\hline & 5 & 1.4991 & 1.4300 & 1.5210 & $1.3900^{* *}$ & $1.3899^{* *}$ & $1.3962^{* *}$ & $1.3908^{* *}$ & $1.3894^{* *}$ \\
\hline & 10 & $1.4746^{* *}$ & $1.4403^{* *}$ & 1.4991 & $1.4297^{* *}$ & $1.4299^{* *}$ & $1.4362^{* *}$ & $1.4335^{* *}$ & $1.4220^{* *}$ \\
\hline & 22 & $1.4940^{* *}$ & $1.4886^{* *}$ & 1.5511 & $1.4867^{* *}$ & $1.4876^{* *}$ & $1.4864^{* *}$ & $1.4886^{* *}$ & $1.4839^{* *}$ \\
\hline & 44 & $1.5822^{* *}$ & $1.5847^{* *}$ & 1.6332 & $1.5589^{* *}$ & $1.5591^{* *}$ & $1.5622^{* *}$ & $1.5597^{* *}$ & $1.5555^{* *}$ \\
\hline & 66 & $1.6453^{*}$ & 1.6520 & 1.8056 & $1.6201^{* *}$ & $1.6211^{* *}$ & $1.6010^{* *}$ & $1.6206^{* *}$ & $1.5970^{* *}$ \\
\hline \multirow{6}{*}{ MAPE } & 1 & 0.6271 & 0.6143 & 0.6204 & $0.5698^{* *}$ & 0.5721 & $0.5689^{* *}$ & $0.5682^{* *}$ & 0.5744 \\
\hline & 5 & 0.6329 & 0.6286 & 0.6371 & $0.6072^{*}$ & 0.6081 & $0.6047^{* *}$ & $0.6078^{*}$ & 0.6090 \\
\hline & 10 & $0.6202^{*}$ & 0.6225 & 0.6364 & $0.6185^{*}$ & 0.6192 & $0.6153^{* *}$ & 0.6204 & 0.6214 \\
\hline & 22 & $0.6355^{* *}$ & $0.6423^{* *}$ & 0.6613 & $0.6405^{* *}$ & $0.6406^{* *}$ & $0.6393^{* *}$ & $0.6409^{* *}$ & 0.6497 \\
\hline & 44 & $0.6733^{* *}$ & $0.6745^{*}$ & 0.7199 & $0.6739^{* *}$ & $0.6745^{*}$ & $0.6762^{*}$ & $0.6744^{*}$ & 0.6887 \\
\hline & 66 & $0.7032^{* *}$ & $0.7190^{* *}$ & 0.7816 & $0.7081^{* *}$ & $0.7088^{* *}$ & $0.7121^{* *}$ & $0.7082^{* *}$ & 0.7286 \\
\hline \multirow{6}{*}{ QLIKE } & 1 & 1.9467 & 1.9167 & 1.9393 & $1.9018^{* *}$ & $1.9018^{* *}$ & $1.9032^{*}$ & $1.9017^{* *}$ & $1.9029^{* *}$ \\
\hline & 5 & 1.9429 & 1.9324 & 1.9494 & $1.9239^{* *}$ & $1.9239^{* *}$ & $1.9258^{*}$ & $1.9244^{* *}$ & $1.9234^{* *}$ \\
\hline & 10 & $1.9389^{*}$ & $1.9328^{* *}$ & 1.9499 & $1.9341^{* *}$ & $1.9340^{* *}$ & $1.9366^{*}$ & $1.9347^{* *}$ & $1.9324^{* *}$ \\
\hline & 22 & $1.9574^{*}$ & $1.9544^{*}$ & 1.9915 & $1.9516^{*}$ & $1.9518^{*}$ & $1.9524^{*}$ & $1.9521^{*}$ & $1.9472^{* *}$ \\
\hline & 44 & 2.0036 & 2.0034 & 2.0022 & 1.9884 & 1.9880 & 1.9875 & 1.9884 & $1.9776^{* *}$ \\
\hline & 66 & 2.0194 & 2.0083 & 2.0441 & $2.0038^{*}$ & $2.0032^{*}$ & $1.9952^{*}$ & $2.0036^{*}$ & $1.9897^{* *}$ \\
\hline \multirow{6}{*}{ SR } & 1 & $0.7124^{* * *}$ & $0.6805^{* * *}$ & $0.7143^{* * *}$ & $0.7105^{* * *}$ & $0.7077^{* * *}$ & $0.7049^{* * *}$ & $0.7180^{* * *}$ & $0.7068^{* * *}$ \\
\hline & 5 & $0.6513^{* * *}$ & $0.6344^{* * *}$ & $0.6560^{* * *}$ & $0.6786^{* * *}$ & $0.6739^{* * *}$ & $0.6842^{* * *}$ & $0.6795^{* * *}$ & $0.6880^{* * *}$ \\
\hline & 10 & $0.6692^{* * *}$ & $0.6363^{* * *}$ & $0.6541^{* * *}$ & $0.6673^{* * *}$ & $0.6692^{* * *}$ & $0.6729^{* * *}$ & $0.6664^{* * *}$ & $0.6758^{* * *}$ \\
\hline & 22 & $0.6654^{* * *}$ & $0.6297^{* * *}$ & $0.6062^{* * *}$ & $0.6288^{* * *}$ & $0.6269^{* * *}$ & $0.6457^{* * *}$ & $0.6269^{* * *}$ & $0.6598^{* * *}$ \\
\hline & 44 & $0.6071^{* * *}$ & $0.5470^{* * *}$ & 0.4643 & $0.5695^{* * *}$ & $0.5714^{* * *}$ & $0.5874^{* * *}$ & $0.5705^{* * *}$ & $0.6006^{* * *}$ \\
\hline & 66 & $0.5536^{* * *}$ & 0.4464 & 0.4502 & 0.4812 & 0.4887 & 0.4887 & 0.4868 & 0.5122 \\
\hline
\end{tabular}

Table 16: Forecasting Evaluation for Wheat Futures with $R V$. Note that $*$ and ** indicate the inclusion in the $\mathcal{M}_{90 \%}^{*}$ and $\mathcal{M}_{75 \%}^{*}$, respectively. For the Success Ratio (SR), the asterisk *, **, and *** indicate a statistical significance at $10 \%, 5 \%$, and $1 \%$, respectively. 


\section{Value-at-Risk backtesting results}

To further demonstrate the economic usefulness of the HAR model and its extensions in risk management applications, we calculate the Value-at-Risk $(V a R)$ for the level $\alpha$ and the forecasting horizon $h$-days ahead by

$$
\operatorname{VaR}_{t+h, \alpha}^{(h)}=\mathrm{RV}_{t+h}^{(h)} z_{\alpha}
$$

where $z_{\alpha}$ is the $\alpha$-quantile of the Standard Normal distribution. Based on this $\operatorname{VaR}$ forecast, we backtest the performance of the individual models using the unconditional coverage test by Kupied (19.95) and the conditional coverage test by Christoffersen (1998). Coverage means that for example a $99 \% \mathrm{VaR}$ should have $1 \%$ violations. A violation occurs if the actual returns exceeds the $V a R$ prediction (long) or turns out to be below the $\operatorname{VaR}$ forecast (short). Both tests compare the actual coverage with the theoretical coverage. While the Kupied (1995) test assumes the violations to be independent from each other, the Christoffersen (1998) test has the alternative hypothesis of a first-order Markov chain.

The $V a R$ results are available in Appendix 0. Evidently, they further confirm that the HAR extensions do not offer any material benefits in a risk management exercise relatively to the simple HAR. Hence, our initial conclusion that the jump component, the continuous component, the signed jumps, and the volatility or return leverage do not offer any significant and economically useful forecasting gains, remains robust.

\section{Conclusion}

The aim of this paper is to add to the extreme scarce literature on agricultural commodities volatility forecasting. Existing studies concentrate their attention to the Chinese futures markets and the benefit of developing model averaging framework. By contrast, they have not focused on the U.S. market, which is the most established market and they do not provide a clear answer as to whether specific volatility components, e.g. the jump component, the continuous component, the signed jumps and the volatility or return leverage can provide incremental predictive gains. Finally, the current literature provides evidence solely based on the realized volatility measure. This study fills these voids by utilizing naive models (Random Walk, AR, ARMA) and several extensions of the simple HAR model (the simple HAR, HAR-J, HAR-CJ, HAR-PS and LHAR-CJ) to forecast two different realized volatility measures, namely, the realized volatility $(R V)$ and median realized volatility $(M e d R V)$. For our study we obtain tick-by-tick data from five important agricultural commodities, i.e. Corn, Rough Rice, Soybeans, Sugar and Wheat and we produce forecasts for 1-day to 66-days ahead. The period of study spans from January 4, 2010 to June 30, 2017 and our out-of-sample period is January 2, 2013 to June $30,2017$. 
In our in-sample analysis, we show that the variants of the HAR model, which decompose the volatility measures into their continuous path and jump components, provide better fitting in the predictive regressions. However, the real out-of-sample forecasts strongly suggest that such decomposition does not offer any superior predictive ability, since none of the variants of the HAR model produce significantly better forecasts compared to the simple HAR model. Thus, there is no benefit to add more complexity in the forecasting models that relates to the volatility decomposition or its relative transformations. Such finding holds for both the $R V$ and $M e d R V$, hence they are not specific to the volatility measure. We note that our findings hold for U.S. markets while other studies, e.g. on Chinese futures markets (Tian et al., 2017a,b, Yang et al., 2017), find increased predictive power of jumps, structural breaks, and time variation of HAR coefficients. We conclude that differing driving factors, such as motive and structure of market participants, could potentially affect differently the behaviour of intra-day volatility and subsequently, its forecastability.

Hence, we maintain that the search for improving the forecasting accuracy of the U.S. agricultural commodities volatility should not be located at the development of extended HAR models that take into account properties such as jump component, the continuous component, the signed jumps and the volatility or return leverage, but rather on other direction, such as the inclusion of exogenous predictors. Degiannakis \& Filis (2018), Degiannakis \& Filis (2017), and Nguyen \& Walther (2019) have already shown that the incorporation of different asset classes volatilities helps improving commodities prices and volatilities (oil prices and volatility in particular) and hence, further study should assess whether such asset classes could also help improve forecasts for agricultural commodities. Even more, future research should consider how extreme weather events, food stocks, biofuels production or even market speculative activity could improve further the agricultural commodity volatility forecasts. Finally, an interesting avenue of further research would be the forecasting accuracy evaluation of alternative forecasting methods, such as machine learning. 


\section{References}

Anderluh, J., \& Borovkova, S. (2008). Commodity volatility modelling and option pricing with a potential function approach. The European Journal of Finance, 14, 91-113. doi:10.1080/13518470701773593.

Andersen, T. G., \& Bollerslev, T. (1998). Answering the Skeptics: Yes, Standard Volatility Models do Provide Accurate Forecasts. International Economic Review, 39, 885905.

Andersen, T. G., Bollerslev, T., \& Diebold, F. X. (2007). Roughing It Up: Including Jump Components in the Measurement, Modeling, and Forecasting of Return Volatility. Review of Economics and Statistics, 89, 701-720. doi:10.1162/rest.89.4.701.

Andersen, T. G., Dobrev, D., \& Schaumburg, E. (2012). Jump-robust volatility estimation using nearest neighbor truncation. Journal of Econometrics, 169, 75-93. doi:10.1016/ j.jeconom.2012.01.011.

Barndorff-Nielsen, O. E., Kinnebrock, S., \& Shephard, N. (2010). Measuring Downside Risk Realized Semivariance. In Volatility and Time Series Econometrics (pp. 117-136). Oxford University Press. doi:10.1093/acprof : oso/9780199549498.003.0007.

Barndorff-Nielsen, O. E., \& Sheppard, N. (2004). Power and Bipower Variation with Stochastic Volatility and Jumps. Journal of Financial Econometrics, 2, 1-37. doi:10. 1093/jjfinec/nbh001.

Bloomberg (2019). There Are Three New Ways to Bet on Chinese Commodity Markets. 28 January 2019, . URL: https://www.bloomberg.com/news/articles/2019-01-28/ there-are-three-new-ways-to-bet-on-chinese-commodity-markets.

von Braun, J., \& Tadesse, G. (2012). Food security, commodity price volatility and the poor. In M. Aoki, T. Kuran, \& G. Roland (Eds.), Institutions and Comparative Economic Development. Palgrave Macmillan Publications, IAE Conference Volume 2012.

CFR (2011). Food Price Volatility and Insecurity. Technical Report. URL: https: //wWw.cfr.org/backgrounder/food-price-volatility-and-insecurity.

Christoffersen, P. F. (1998). Evaluating Interval Forecasts. International Economic Review, 39, 841-862. doi:10.2307/2527341.

Corsi, F. (2009). A simple approximate long-memory model of realized volatility. Journal of Financial Econometrics, 7, 174-196. doi:10.1093/jjfinec/nbp001.

Corsi, F., \& Renò, R. (2012). Discrete-Time Volatility Forecasting With Persistent Leverage Effect and the Link With Continuous-Time Volatility Modeling. Journal of Business \& Economic Statistics, 30, 368-380. doi:10.1080/07350015.2012.663261.

Degiannakis, S. (2008). ARFIMAX and ARFIMAX-TARCH realized volatility modeling. Journal of Applied Statistics, 35, 1169-1180. doi:10.1080/02664760802271017.

Degiannakis, S., \& Filis, G. (2017). Forecasting oil price realized volatility using information channels from other asset classes. Journal of International Money and Finance, 76, 28-49. doi:10.1016/j·jimonfin.2017.05.006. 
Degiannakis, S., \& Filis, G. (2018). Forecasting oil prices: High-frequency financial data are indeed useful. Energy Economics, 76, 388-402. doi:10.1016/j.eneco.2018.10. 026.

Degiannakis, S., Filis, G., \& Hassani, H. (2018). Forecasting global stock market implied volatility indices. Journal of Empirical Finance, 46, 111-129. doi:10.1016/j.jempfin. 2017.12 .008 .

Ederington, L. H., \& Guan, W. (2010). Longer-term time-series volatility forecasts. Journal of Financial and Quantitative Analysis, 45, 1055-1076. doi:10.1017/ S0022109010000372.

Egelkraut, T. M., \& Garcia, P. (2006). Intermediate Volatility Forecasts Using Implied Forward Volatility: The Performance of Selected Agricultural Commodity Options. Journal of Agricultural and Resource Economics Journal of Agricultural and Resource Economics, 31, 508-528.

Elder, J., \& Jin, H. J. (2007). Long memory in commodity futures volatility: A wavelet perspective. Journal of Futures Markets, 27, 411-437. doi:10.1002/fut.20260.

Engle, R., Hong, C.-H., \& Kane, A. (1990). Valuation of Variance Forecast with Simulated Option Markets. Technical Report National Bureau of Economic Research Cambridge, MA. doi:10.3386/w3350.

Fan, J. H., \& Zhang, T. (2018). Demystifying Commodity Futures in China. SSRN Electronic Journal, . URL: https://wWw.ssrn.com/abstract=3124223. doi:10.2139/ ssrn. 3124223 .

FAO (2010). Price Volatility in Agricultural Markets Evidence, impact on food security and policy responses. Technical Report. URL: http://www.fao.org/docrep/013/ am053e/am053e00.pdf.

FAO (2011). Price Volatility in Food and Agricultural Markets: Policy Responses. Technical Report. URL: http://www.fao.org/fileadmin/templates/est/Volatility/ Interagency_Report_to_the_G20_on_Food_Price_Volatility.pdf.

Giot, P., \& Laurent, S. (2003). Market risk in commodity markets: a VaR approach. Energy Economics, 25, 435-457. doi:10.1016/S0140-9883(03)00052-5.

Greb, F., \& Prakash, A. (2015). Has price volatility changed? Food Outlook. Food and Agriculture Organization of the United Nations, Rome, Italy, .

Greb, F., \& Prakash, A. (2017). Assessing volatility patterns in food crops. URL: http: //www.fao.org/3/a-i7066e.pdf.

Hansen, P. R., Lunde, A., \& Nason, J. M. (2011). The Model Confidence Set. Econometrica, 79, 453-497. doi:10.3982/ECTA5771.

Huang, H.-C. R. (2004). A flexible nonlinear inference to the Kuznets hypothesis. Economics Letters, 84, 289-296. doi:10.1016/j.econlet.2004.01.014.

Klein, T., \& Todorova, N. (2018). The Impact of SHFE's Night Trading Session on Volume and Realized Volatility of Aluminum and Copper Futures Markets. SSRN Electronic Journal - Working Paper, (pp. 1-29). URL: https://www.ssrn.com/abstract= 3249598. doi:10.2139/ssrn.3249598. 
Kupiec, P. H. (1995). Techniques for Verifying the Accuracy of Risk Measurement Models. The Journal of Derivatives, 3, 73-84. doi:10.3905/jod.1995.407942.

Li, N., Ker, A., Sam, A. G., \& Aradhyula, S. (2017). Modeling regime-dependent agricultural commodity price volatilities. Agricultural Economics, 48, 683-691. doi:10.1111/ agec.12366.

Liu, L. Y., Patton, A. J., \& Sheppard, K. (2015). Does anything beat 5-minute RV? A comparison of realized measures across multiple asset classes. Journal of Econometrics, 187, 293-311. doi:10.1016/j.jeconom.2015.02.008.

Luo, J., Klein, T., Ji, Q., \& Hou, C. (2019). Forecasting Realized Volatility of Agricultural Commodity Futures with Infinite Hidden Markov HAR Models. International Journal of Forecasting, forth.

Nguyen, D. K., \& Walther, T. (2019). Modeling and Forecasting Commodity Market Volatility with Long-term Economic and Financial Variables. Journal of Forecasting, forth. doi:10.1002/for.2617.

Ordu, B. M., Oran, A., \& Soytas, U. (2018). Is food financialized? Yes, but only when liquidity is abundant. Journal of Banking \& Finance, 95, 82-96. doi:10.1016/j. jbankfin.2017.06.001.

Patton, A. J. (2011). Volatility forecast comparison using imperfect volatility proxies. Journal of Econometrics, 160, 246-256. doi:10.1016/j.jeconom.2010.03.034.

Patton, A. J., \& Sheppard, K. (2015). Good Volatility, Bad Volatility: Signed Jumps and The Persistence of Volatility. Review of Economics and Statistics, 97, 683-697. doi:10.1162/REST\{\_\}a\{\_\}00503.

Pesaran, M. H., \& Timmermann, A. (1992). A Simple Nonparametric Test of Predictive Performance. Journal of Business \& Economic Statistics1, 10, 461-465. doi:10.1080/ 07350015.1992 .10509922$.

Sévi, B. (2014). Forecasting the volatility of crude oil futures using intraday data. European Journal of Operational Research, 235, 643-659. doi:10.1016/j.ejor.2014.01. 019 .

Theodosiou, M., \& Zikes, P. (2011). A comprehensive comparison of alternative tests for jumps in asset prices. Working Paper, Central Bank of Cyprus, .

Tian, F., Yang, K., \& Chen, L. (2017a). Realized Volatility Forecasting of Agricultural Commodity Futures Using Long Memory and Regime Switching. Journal of Forecasting , 36, 421-430. doi:10.1002/for.2443.

Tian, F., Yang, K., \& Chen, L. (2017b). Realized volatility forecasting of agricultural commodity futures using the HAR model with time-varying sparsity. International Journal of Forecasting, 33, 132-152. doi:10.1016/j.ijforecast.2016.08.002.

Triantafyllou, A., Dotsis, G., \& Sarris, A. H. (2015). Volatility Forecasting and Timevarying Variance Risk Premiums in Grains Commodity Markets. Journal of Agricultural Economics, 66, 329-357. doi:10.1111/1477-9552.12101.

Yang, K., Tian, F., Chen, L., \& Li, S. (2017). Realized volatility forecast of agricultural futures using the HAR models with bagging and combination approaches. International Review of Economics \& Finance, 49, 276-291. doi:10.1016/j.iref.2017.01.030. 
Zhang, D. (2017). Oil shocks and stock markets revisited: Measuring connectedness from a global perspective. Energy Economics, 62, 323-333. doi:10.1016/j .eneco.2017.01. 009 . 
Appendix A. Results MedRV

Appendix A.1. In-Sample 


\begin{tabular}{|c|c|c|c|c|c|}
\hline$h$ & 1 & 5 & 10 & 22 & 44 \\
\hline & & & dom Walk & & \\
\hline adj. $R^{2}$ & 0.1393 & 0.3561 & 0.2977 & 0.1964 & -0.0923 \\
\hline $\begin{array}{l}\text { MSPE } \\
\text { MAPE }\end{array}$ & $\begin{array}{l}1.3701 \\
0.4198\end{array}$ & $\begin{array}{l}1.3857 \\
0.4713^{* *}\end{array}$ & $\begin{array}{c}1.4405 \\
0.5074^{* *}\end{array}$ & $\begin{array}{l}1.4957 \\
0.5431 * *\end{array}$ & $\begin{array}{l}1.6236 \\
0.6535\end{array}$ \\
\hline QLIKE & $1.7198^{* *}$ & $1.8561^{* *}$ & $1.9108^{* *}$ & $1.9650^{* *}$ & $2.0696^{* *}$ \\
\hline & & & $\mathrm{AR}(1)$ & & \\
\hline c & $\begin{array}{c}0.9108 \\
(12.6454)\end{array}$ & $\begin{array}{c}0.6785 \\
(12.8731)\end{array}$ & $\begin{array}{c}0.7409 \\
(15.5199)\end{array}$ & $\begin{array}{c}0.8426 \\
(16.0548)\end{array}$ & $\begin{array}{c}1.1508 \\
(23.9994)\end{array}$ \\
\hline & .5696 & 0.6779 & 0.6487 & 0.5987 & 0.4528 \\
\hline$R V^{(n)}$ & $(14.6496)$ & (23.5169) & $(24.9195)$ & $(21.2109)$ & $(19.2723)$ \\
\hline adj. $R^{2}$ & 0.3232 & 0.4592 & 0.4203 & 0.3557 & 0.1998 \\
\hline MSPE & 1.2143 & $1.3108^{* *}$ & $1.3648^{* *}$ & 1.4170 & 1.4935 \\
\hline APE & $\begin{array}{l}0.4378 \\
1.9448\end{array}$ & $\begin{array}{l}0.4805^{*} \\
1.9707\end{array}$ & $\begin{array}{c}0.5134^{* *} \\
20128\end{array}$ & $\begin{array}{l}0.5449^{* *} \\
20504\end{array}$ & $\begin{array}{l}0.6121 \\
2.1212^{* *}\end{array}$ \\
\hline & & & ARMA & & \\
\hline$c$ & 0.1551 & 0.4081 & 0.5141 & 0.8943 & 1.7898 \\
\hline$c^{c}$ & $(5.4775)$ & $(8.0043)$ & $(9.0119)$ & (10.6561) & $(16.5183)$ \\
\hline$R V^{(h)}$ & $\begin{array}{c}0.9265 \\
(102.6925)\end{array}$ & $\begin{array}{c}0.8066 \\
(44.4168)\end{array}$ & $\begin{array}{c}0.7563 \\
(37.3471)\end{array}$ & $\begin{array}{l}0.5746 \\
(17.5697)\end{array}$ & $\begin{array}{r}0.1533 \\
(3.3155)\end{array}$ \\
\hline & -0.6266 & -0.2488 & -0.1897 & 0.0399 & 0.4086 \\
\hline$\varepsilon^{(h)}$ & $(38.7335)$ & $(9.7088)$ & $(7.0618)$ & $(0.9944)$ & $(8.6762)$ \\
\hline adj. $R^{2}$ & 0.3899 & 0.4711 & 0.4285 & 0.3580 & 0.2410 \\
\hline $\begin{array}{l}\text { MSPE } \\
\text { MAPE }\end{array}$ & $\begin{array}{c}1.1536^{* * *} \\
0.4002\end{array}$ & $\begin{array}{l}1.3011^{* * *} \\
0.472^{* * *}\end{array}$ & $\begin{array}{l}1.3615^{* *} \\
0.5084^{* *}\end{array}$ & $\begin{array}{l}1.4156^{*} \\
0.5435^{* *}\end{array}$ & $\begin{array}{l}1.4830 \\
0.6021\end{array}$ \\
\hline & 1.8943 & 1.9625 & 2.0070 & 2.0487 & $2.1111^{* *}$ \\
\hline & & & HAR & & \\
\hline c & 0.3098 & 0.4695 & 0.6129 & 0.8049 & 1.0913 \\
\hline$V^{(1)}$ & 0.2878 & $\begin{array}{l}(9.11301) \\
0.1601\end{array}$ & 0.1381 & 0.0910 & 0.0637 \\
\hline$R V(1)$ & (5.9968) & $(5.8801)$ & $(5.2162)$ & $(3.6860)$ & $(2.9905)$ \\
\hline$R V^{(5)}$ & $\begin{array}{l}0.3435 \\
(4.7716\end{array}$ & $\begin{array}{l}0.3542 \\
(7.586)\end{array}$ & $\begin{array}{r}0.2710 \\
(6.3246\end{array}$ & 0.2089 & 0.1930 \\
\hline & (4.7716) & $(7.5856)$ & $(6.3246)$ & $(5.0017)$ & (5.4903) \\
\hline$R V^{(22)}$ & $\begin{array}{l}0.2222 \\
(3.5670)\end{array}$ & $\begin{array}{l}0.2620 \\
(6.4302)\end{array}$ & $\begin{array}{c}0.2996 \\
(7.4439)\end{array}$ & $\begin{array}{l}0.3173 \\
(7.7915)\end{array}$ & $\begin{array}{l}0.2263 \\
(6.8787)\end{array}$ \\
\hline adj. $R^{2}$ & 0.3933 & 0.4930 & 0.4660 & 0.4165 & 0.3234 \\
\hline $\begin{array}{l}\text { MSPE } \\
\text { MAPE }\end{array}$ & $1.1491^{* * *}$ & $\begin{array}{l}1.2997^{* *} \\
0.469 * *\end{array}$ & $\begin{array}{l}1.3663^{* *} \\
0.5025^{* *}\end{array}$ & $\begin{array}{l}1.4146 \\
0.5411^{* *}\end{array}$ & $\begin{array}{l}1.4871 \\
0.5977\end{array}$ \\
\hline $\begin{array}{l}\text { MAPE } \\
\text { QLIKE }\end{array}$ & $\begin{array}{l}.39889 \\
1.8889\end{array}$ & $\begin{array}{c}0.4695 \\
1.9556\end{array}$ & $\begin{array}{c}0.50255^{*} \\
1.9957\end{array}$ & $\begin{array}{c}0.5411 * * \\
2.0413\end{array}$ & $\begin{array}{c}0.5977 * \\
2.0986^{* *}\end{array}$ \\
\hline & & & HAR-J & & \\
\hline c & 0.2833 & 0.4567 & 0.6010 & 0.7983 & 1.0821 \\
\hline & $\begin{array}{c}(3.4808) \\
0.2649\end{array}$ & $\begin{array}{l}(8.9205) \\
0.1491\end{array}$ & $\begin{array}{c}(12.7789) \\
0.1280\end{array}$ & $\begin{array}{c}(16.4661) \\
0.0855\end{array}$ & $\begin{array}{l}(25.5415) \\
0.0559\end{array}$ \\
\hline$R V^{(1)}$ & $(5.3369)$ & $(5.3565)$ & $(4.7597)$ & $(3.3782)$ & $(2.5703)$ \\
\hline$R V^{(5)}$ & $\begin{array}{l}0.3266 \\
(4.7009)\end{array}$ & 0.3461 & 0.2635 & 0.2048 & 0.1872 \\
\hline & $(4.7099)$ & $(7.3960)$ & (6.1448) & $(4.9089)$ & (5.3058) \\
\hline$R V^{(22)}$ & $\begin{array}{l}0.2240 \\
(3.6098)\end{array}$ & $\begin{array}{l}0.2629 \\
(6.4700)\end{array}$ & $\begin{array}{r}0.3005 \\
(7.4972)\end{array}$ & $\begin{array}{l}0.3178 \\
(7.8372)\end{array}$ & $\begin{array}{l}0.2270 \\
(6.9554)\end{array}$ \\
\hline$J^{(1)}$ & 0.2621 & 0.1263 & 0.1156 & 0.0638 & 0.0899 \\
\hline$J(1)$ & $(2.3993)$ & $(2.3183)$ & $(2.5983)$ & $(1.6828)$ & $(2.2638)$ \\
\hline adj. $R^{2}$ & 0.4000 & 0.4954 & 0.4684 & 0.4172 & 0.3255 \\
\hline $\begin{array}{l}\text { MSPE } \\
\text { MAPE }\end{array}$ & $\begin{array}{l}1.1425^{* * *} \\
0.3912^{* *}\end{array}$ & $\begin{array}{l}1.3000^{* *} \\
0.469^{* *}\end{array}$ & $\begin{array}{l}1.3677^{* *} \\
0.5026^{* *}\end{array}$ & $\begin{array}{l}1.4152 \\
0.5404^{* *}\end{array}$ & $\begin{array}{l}1.4857 \\
0.5966\end{array}$ \\
\hline & 1.8838 & 1.9545 & 1.9952 & 2.0406 & $2.0973^{* *}$ \\
\hline & & & IAR-CJ & & \\
\hline c & $\begin{array}{l}0.3686 \\
(4.7122)\end{array}$ & $\begin{array}{l}0.5305 \\
(0.6153)\end{array}$ & 0.6808 & 0.9030 & 1.2085 \\
\hline$J^{(1)}$ & $\begin{array}{c}(4.7122) \\
0.1626\end{array}$ & $\begin{array}{c}(10.6153) \\
0.0887\end{array}$ & $\begin{array}{c}(14.6331) \\
0.0941\end{array}$ & $\begin{array}{c}(18.67377) \\
0.0551\end{array}$ & $\begin{array}{c}(28.9429) \\
0.0361\end{array}$ \\
\hline$J^{(1)}$ & $(1.6227)$ & $(1.6277)$ & $(2.2697)$ & (1.6383) & $(0.9978)$ \\
\hline$J^{(5)}$ & $\begin{array}{c}0.0777 \\
(2.0670)\end{array}$ & $\begin{array}{l}0.0875 \\
(3.6559)\end{array}$ & $\begin{array}{l}0.0422 \\
(2.0600)\end{array}$ & $\begin{array}{c}-0.0148 \\
(-0.7519)\end{array}$ & $\begin{array}{l}0.0001 \\
(0.0036)\end{array}$ \\
\hline$J^{(22)}$ & -0.0025 & 0.0006 & 0.0179 & 0.0522 & 0.0722 \\
\hline & $(-0.2020)$ & $(0.0622)$ & $(2.3165)$ & $(7.2854)$ & (10.6087) \\
\hline$C^{(1)}$ & $\begin{array}{l}0.2649 \\
(6.5784)\end{array}$ & $\begin{array}{l}0.1837 \\
(5.7244)\end{array}$ & $\begin{array}{r}0.1199 \\
(5.1117)\end{array}$ & $\begin{array}{l}0.0820 \\
(3.7994)\end{array}$ & $\begin{array}{c}0.0578 \\
(3.2770)\end{array}$ \\
\hline$C^{(5)}$ & 0.2624 & 0.2845 & 0.2297 & 0.1871 & 0.1613 \\
\hline & $(4.2929)$ & $(6.8340)$ & $(6.1022)$ & $(5.2061)$ & $(5.6649)$ \\
\hline$C^{(22)}$ & $\begin{array}{c}0.2042 \\
(3.6547)\end{array}$ & $\begin{array}{l}0.2316 \\
(5.8049)\end{array}$ & $\begin{array}{c}0.2251 \\
(5.8277)\end{array}$ & $\begin{array}{l}0.1707 \\
(4.7512)\end{array}$ & $\begin{array}{c}0.0445 \\
(1.6043)\end{array}$ \\
\hline adj. $R^{2}$ & 0.4010 & 0.5003 & 0.4761 & 0.4378 & 0.3867 \\
\hline MSPE & $1.1408^{* *}$ & $1.2947^{* *}$ & $1.3632^{* *}$ & $1.3967^{* *}$ & 1.4567 \\
\hline $\begin{array}{l}\text { MAPE } \\
\text { OLIKE }\end{array}$ & $\begin{array}{l}0.3901^{* * *} \\
1.8833\end{array}$ & $\begin{array}{l}0.4684^{* *} \\
1.938\end{array}$ & $\begin{array}{l}0.5015^{* *} \\
1.9935\end{array}$ & $0.5332^{* *}$ & $\begin{array}{c}0.5800 \\
20820^{* *}\end{array}$ \\
\hline & 1.8833 & 1.9538 & 1.9935 & 2.0332 & $2.0829^{* *}$ \\
\hline & & & IAR-PS & & \\
\hline c & $\begin{array}{l}0.3165 \\
(39645\end{array}$ & $\begin{array}{l}0.4761 \\
(9.3418)\end{array}$ & 0.6162 & 0.8040 & 1.0879 \\
\hline & $\begin{array}{c}(3.9645) \\
0.1467\end{array}$ & $\begin{array}{c}(9.3418) \\
0.1049\end{array}$ & $\begin{array}{c}\left(\begin{array}{c}(3.0527) \\
0.0755\end{array}\right) \\
0\end{array}$ & $\begin{array}{c}(16.7068) \\
0.0333\end{array}$ & $\begin{array}{c}\left(\begin{array}{c}26.0791) \\
0.0237\end{array}\right) \\
0\end{array}$ \\
\hline$R S^{+}$ & $(2.3957)$ & $(3.1172)$ & $(2.6586)$ & $(1.2713)$ & $(0.8861)$ \\
\hline$R S^{-}$ & 0.4074 & 0.1890 & 0.1874 & 0.1653 & 0.1504 \\
\hline & $(4.7053)$ & $(3.7896)$ & $(3.9352)$ & $(3.8112)$ & $(4.0479)$ \\
\hline $\mathbf{I}_{r_{t}<0} R V^{(1)}$ & $\begin{array}{l}-0.0389 \\
(-0.9204)\end{array}$ & $\begin{array}{l}0.0003 \\
(0.006)\end{array}$ & $\begin{array}{c}0.0002 \\
(0.0082)\end{array}$ & $\begin{array}{c}-0.0301 \\
(-1.3133)\end{array}$ & $\begin{array}{c}-0.0383 \\
(-1.9616)\end{array}$ \\
\hline$R V^{(5)}$ & 0.3354 & 0.3451 & 0.2584 & 0.2032 & 0.1781 \\
\hline & (5.0532) & $(7.4672)$ & $(6.2539)$ & $(5.0904)$ & $(5.2445)$ \\
\hline$R V^{(22)}$ & 0.214 & 0.2582 & 0.2967 & 0.3150 & 0.2252 \\
\hline & .4t93) & (6.3739) & $(7.4231)$ & $(7.8520)$ & $(6.9884)$ \\
\hline adj. $R^{2}$ & $\begin{array}{l}0.4054 \\
0\end{array}$ & 0.4986 & 0.4736 & 0.4217 & 0.3309 \\
\hline $\begin{array}{l}\text { MSPE } \\
\text { MAPE }\end{array}$ & $\begin{array}{l}1.1369^{* *} \\
0.3907^{* *}\end{array}$ & $\begin{array}{l}1.3007 \% * \\
0.4691^{* *}\end{array}$ & $\begin{array}{l}1.3663^{* *} \\
0.5025^{* *}\end{array}$ & $\begin{array}{l}1.4139 \% \\
0.5388^{* *}\end{array}$ & $\begin{array}{l}1.4837 \\
0.5949\end{array}$ \\
\hline QLIKE & 1.8816 & 1.9535 & 1.9942 & 2.0383 & $2.0958^{* *}$ \\
\hline & & & HAR-CJ & & \\
\hline $\mathrm{c}$ & 0. & (9. & $\begin{array}{r}0.653 \\
0.59\end{array}$ & 0.9 & 1.2 \\
\hline$J^{(1)}$ & & & & & \\
\hline J1) & (1.5935) & (1.5699) & $\begin{array}{l}0.0880 \\
(2.1747)\end{array}$ & $\begin{array}{l}0.0499 \\
(1.5103)\end{array}$ & $\begin{array}{l}0.0300 \\
(0.8602)\end{array}$ \\
\hline$J^{(5}$ & $\begin{array}{c}0.0770 \\
(2.0719)\end{array}$ & $\begin{array}{l}0.0863 \\
(3643)\end{array}$ & $\begin{array}{l}0.0412 \\
(20238)\end{array}$ & -0.0151 & 0.0000 \\
\hline & -0.6 r. & $\begin{array}{l}(3.6433) \\
-0.0014\end{array}$ & $\begin{array}{c}(2.0238) \\
0.0171\end{array}$ & $\begin{array}{c}(-0.7721) \\
0.0533\end{array}$ & $(0.0014)$ \\
\hline$J^{(2}$ & $\begin{array}{r}-0.00 \\
(-0.32\end{array}$ & $\begin{array}{l}-0.0014 \\
(-0.1498)\end{array}$ & $\begin{array}{r}0.1715 \\
(2.1515\end{array}$ & $\begin{array}{l}0.0533 \\
(7.2455)\end{array}$ & $\begin{array}{c}0.0747 \\
(10.9309)\end{array}$ \\
\hline & & 0.1 & 0.16 & 0.0 & 0.05 \\
\hline C & $\begin{array}{l}0.24 \\
(6.22\end{array}$ & $\begin{array}{l}0.12 \\
(5.42\end{array}$ & $(4.76$ & $(3.4$ & \\
\hline$C^{(5)}$ & 0.2658 & 0.2832 & 0.2342 & 0.1 & 0.1793 \\
\hline C & (4.3407) & $(6.8237)$ & $(6.17$ & $(5.4$ & \\
\hline$C^{(22)}$ & $\begin{array}{c}0.2127 \\
(3.8489)\end{array}$ & 0.2397 & 0.2275 & 0.1636 & 0.02 \\
\hline & (3.8489) & (5.9377) & (5.8137) & $(4.5265)$ & (1.0783) \\
\hline$r_{t}^{-,(1)}$ & $\begin{array}{l}-0.1069 \\
(-2.5449)\end{array}$ & $\begin{array}{l}-0.0 \\
-2.6 \\
-6\end{array}$ & -0.0697 & $\begin{array}{l}-0.0482 \\
(-25132)\end{array}$ & -0.0482 \\
\hline & & 0.0 & -3.1 & $(-2.5$ & $(-2.8)$ \\
\hline & $\begin{array}{l}-0.0029 \\
(-0.4766)\end{array}$ & $(1.4119)$ & $\begin{array}{c}0.0055 \\
(1.1699)\end{array}$ & $\begin{array}{l}0.0028 \\
(0.6467)\end{array}$ & 0.0013 \\
\hline & $\begin{array}{l}(-0.466) \\
-0.1099\end{array}$ & $\begin{array}{l}(1.4119) \\
-0.0626\end{array}$ & $\begin{array}{c}(1.1699) \\
0.0456\end{array}$ & $\begin{array}{c}(0.0467) \\
0.2018\end{array}$ & $\begin{array}{c}(0.3393) \\
0.3454\end{array}$ \\
\hline$r_{t}$ & $(-0.7278)$ & $\begin{array}{l}-0.0020 \\
(-0.6529)\end{array}$ & $(0.5430)$ & $\begin{array}{l}0.2810 \\
(2.8277)\end{array}$ & $(5.6021)$ \\
\hline 1j. $R^{2}$ & .4052 & 0.503 & 0.4798 & 0.44 & 0.3954 \\
\hline & 1.135 & $1.2949^{* *}$ & $1.3617^{* *}$ & $1.3955^{* *}$ & $1.4501^{* *}$ \\
\hline $\begin{array}{l}\text { MAPE } \\
\text { QLIKE }\end{array}$ & $\begin{array}{c}0.3871 \\
1.8779\end{array}$ & $\begin{array}{l}0.4677^{* *} \\
1.9512\end{array}$ & $\begin{array}{l}0.5009^{* *} \\
1.9915\end{array}$ & $\begin{array}{l}0.53433^{* *} \\
2.0327\end{array}$ & $\begin{array}{l}0.5761^{* *} \\
2.0799^{* *}\end{array}$ \\
\hline
\end{tabular}

Table A.17: In-Sample regression results for Corn with $M e d R V$. Note that * and ** indicate the inclusion in the $\mathcal{M}_{90 \%}^{*}$ and $\mathcal{M}_{75 \%}^{*}$, respectively. The $t$-statistics for the parameter estimates are given in parentheses. 


\begin{tabular}{|c|}
\hline$h$ \\
\hline \\
\hline $\operatorname{adj} . R^{2}$ \\
\hline $\begin{array}{l}\text { MSPE } \\
\text { MAPE }\end{array}$ \\
\hline \\
\hline \\
\hline \\
\hline c \\
\hline$R V^{(h)}$ \\
\hline adj. $R^{2}$ \\
\hline MSPE \\
\hline MAPE \\
\hline QLIKE \\
\hline \\
\hline \\
\hline $\mathrm{c}$ \\
\hline$R V^{(h)}$ \\
\hline \\
\hline$\varepsilon^{(h)}$ \\
\hline adj. $R^{2}$ \\
\hline $\begin{array}{l}\text { MSPE } \\
\text { MAPE }\end{array}$ \\
\hline QLIKE \\
\hline \\
\hline c \\
\hline \\
\hline$R V^{(1)}$ \\
\hline$R V^{(5)}$ \\
\hline \\
\hline$R V^{(22)}$ \\
\hline adj. R \\
\hline MSP \\
\hline \\
\hline QLIKE \\
\hline \\
\hline c \\
\hline$R V^{(1)}$ \\
\hline$R V^{(1)}$ \\
\hline$R V^{(5)}$ \\
\hline \\
\hline$R V^{(22)}$ \\
\hline$J^{(1)}$ \\
\hline$J^{(1)}$ \\
\hline adj. $R^{2}$ \\
\hline $\mathrm{PE}$ \\
\hline \\
\hline QLIKE \\
\hline \\
\hline c \\
\hline \\
\hline$J^{(1)}$ \\
\hline$J^{(5)}$ \\
\hline \\
\hline$J^{(22)}$ \\
\hline$C^{(1)}$ \\
\hline \\
\hline$C^{(5)}$ \\
\hline \\
\hline$C^{(22)}$ \\
\hline adj. $R^{2}$ \\
\hline MSPE \\
\hline $\begin{array}{l}\text { MAPE } \\
\text { QLIKE }\end{array}$ \\
\hline \\
\hline c \\
\hline \\
\hline$R S^{+}$ \\
\hline$R S^{-}$ \\
\hline \\
\hline $\mathbf{I}_{r_{t}<0} R V^{(1)}$ \\
\hline \\
\hline$R V$ \\
\hline$B V^{(22)}$ \\
\hline$R V^{(22)}$ \\
\hline adj. $R^{2}$ \\
\hline MSPE \\
\hline MA \\
\hline QLIKE \\
\hline \\
\hline c \\
\hline \\
\hline$J^{(1)}$ \\
\hline \\
\hline$J^{(5)}$ \\
\hline$J^{(22)}$ \\
\hline \\
\hline$C^{(1)}$ \\
\hline$C^{(5)}$ \\
\hline$C^{(0)}$ \\
\hline$C^{(22)}$ \\
\hline$C(2)$ \\
\hline$r_{t}^{-,(1)}$ \\
\hline \\
\hline$r_{t}^{-,(5)}$ \\
\hline \\
\hline \\
\hline adj. $R^{2}$ \\
\hline SPE \\
\hline P \\
\hline QLI \\
\hline
\end{tabular}

Table A.18: In-Sample regression results for Rough Rice with $M e d R V$. Note that $*$ and $* *$ indicate the inclusion in the $\mathcal{M}_{90 \%}^{*}$ and $\mathcal{M}_{75 \%}^{*}$, respectively. The $t$-statistics for the parameter estimates are given in parentheses. 


\begin{tabular}{|c|c|}
\hline$h$ & 1 \\
\hline & \\
\hline adj. $R^{2}$ & 0.1534 \\
\hline & 0.7996 \\
\hline & $\begin{array}{c}0.7928 \\
1.6033^{* *}\end{array}$ \\
\hline & $1.6033^{* 4}$ \\
\hline & \\
\hline $\begin{array}{l}\text { c } \\
R V^{(h)}\end{array}$ & $\begin{array}{l}0.5462 \\
(9.9397) \\
0.5765\end{array}$ \\
\hline & \\
\hline adj. $R^{2}$ & 0.3318 \\
\hline MSPE & 0.7100 \\
\hline MAPE & 0.8160 \\
\hline QLIKE & 1.8267 \\
\hline & \\
\hline$c$ & 0.1173 \\
\hline & $(6.2369)$ \\
\hline$R V^{(h)}$ & $\begin{array}{l}0.9086 \\
(85.9890)\end{array}$ \\
\hline$\varepsilon^{(h)}$ & $\begin{array}{c}-0.5725 \\
(33.7617)\end{array}$ \\
\hline adj. $R^{2}$ & 0.3956 \\
\hline MSPE & $\begin{array}{l}0.69506^{*} \\
0.67\end{array}$ \\
\hline & $0.7888^{* *}$ \\
\hline QLIK & 1.7927 \\
\hline & \\
\hline c & 0.2019 \\
\hline & $\begin{array}{c}(4.0585) \\
0.3014\end{array}$ \\
\hline$R V^{(1)}$ & $(5.1651)$ \\
\hline$R V^{(5)}$ & $0.3670^{\prime}$ \\
\hline & $\begin{array}{l}(5.6254) \\
0.1745\end{array}$ \\
\hline$R V^{(22)}$ & $\begin{array}{l}0.1745 \\
(2.7301)\end{array}$ \\
\hline adj. $R^{2}$ & 0.3964 \\
\hline $\begin{array}{l}\text { MSPE } \\
\text { MAPE }\end{array}$ & $0.6744^{*}$ \\
\hline $\begin{array}{l}\text { MAP } \\
\text { QLIK }\end{array}$ & $\begin{array}{l}0.7611^{* *} \\
1.7624\end{array}$ \\
\hline & \\
\hline & \\
\hline c & 0.1984 \\
\hline (1) & $\begin{array}{c}(3.9871) \\
0.2979\end{array}$ \\
\hline$R V(1)$ & $(5.0584)$ \\
\hline$R V^{(5)}$ & 0.3655 \\
\hline & $(5.6162)$ \\
\hline$R V^{(22)}$ & $\begin{array}{c}0.1739 \\
(2.7207)\end{array}$ \\
\hline$J^{(1)}$ & 0.0464 \\
\hline$J^{(1)}$ & $(0.9395)$ \\
\hline adj. $R^{2}$ & 0.3964 \\
\hline MSPE & $0.6743^{*}$ \\
\hline $\begin{array}{l}\text { MAPE } \\
\text { QLIKE }\end{array}$ & $\begin{array}{c}0.7606^{* *} \\
1.7616\end{array}$ \\
\hline & \\
\hline c & 0.1818 \\
\hline & $\begin{array}{c}(3.4649) \\
0.0740\end{array}$ \\
\hline$J^{(1)}$ & $(1.7967)$ \\
\hline$J^{(5)}$ & -0.0321 \\
\hline$J^{2}(2)$ & (-1.4070) \\
\hline$J^{(22)}$ & $\begin{array}{l}0.0068 \\
(0.7238)\end{array}$ \\
\hline$C^{(1}$ & 0.2968 \\
\hline & $(6.8981)$ \\
\hline$C^{(5)}$ & 0.3323 \\
\hline & $(6.3988)$ \\
\hline$C^{(22)}$ & $\begin{array}{l}0.1606 \\
(2.8900)\end{array}$ \\
\hline & \\
\hline & $\begin{array}{l}0.60839^{*} \\
0.06\end{array}$ \\
\hline & \\
\hline QLIKE & 1.7109 \\
\hline & \\
\hline c & 0 \\
\hline & \\
\hline$R S^{+}$ & $\begin{array}{l}0.2189 \\
(3.4840)\end{array}$ \\
\hline$R S^{-}$ & 0.2315 \\
\hline & $(3.6797)$ \\
\hline $\mathbf{I}_{r_{t}<0} R V^{(1)}$ & $\begin{array}{c}-0.0075 \\
(-0.1936)\end{array}$ \\
\hline$(5)$ & $\begin{array}{c}(-0.1950) \\
0.4245\end{array}$ \\
\hline$R V^{(5)}$ & $(7.5615)$ \\
\hline$R V^{(22)}$ & 0.1640 \\
\hline & $(2.5335)$ \\
\hline adj. $R^{2}$ & 0.39 \\
\hline & \\
\hline & 0.751 \\
\hline & 1.7516 \\
\hline & \\
\hline c & 0. \\
\hline & \\
\hline$J^{(1)}$ & $\begin{array}{l}0.0518 \\
(1.4033)\end{array}$ \\
\hline$J^{(5)}$ & -0.034 \\
\hline & $(-1$ \\
\hline$J^{(22)}$ & \\
\hline & \\
\hline$C^{(1)}$ & $\begin{array}{l}0.2821 \\
(6.5677)\end{array}$ \\
\hline$a^{(}$ & 0.3293 \\
\hline$C^{c}$ & (6.3106) \\
\hline$C$ & 0.1626 \\
\hline & \\
\hline$r_{t}^{-,(1)}$ & \\
\hline & (-1.9576) \\
\hline$r_{t}^{-,(5)}$ & $\begin{array}{c}0.0073 \\
(1.2940)\end{array}$ \\
\hline$r_{-,(22)}^{-, ~}$ & (1) \\
\hline & $(-0.7749)$ \\
\hline adj. $R^{2}$ & \\
\hline $\begin{array}{l}\text { MSPI } \\
\text { MAP }\end{array}$ & $\begin{array}{l}0.6668^{* *} \\
07711^{* *}\end{array}$ \\
\hline QLIKE & $\begin{array}{l}0.7711 \\
1.7073\end{array}$ \\
\hline
\end{tabular}

Table A.19: In-Sample regression results for Soybean with $M e d R V$. Note that ${ }^{*}$ and $* *$ indicate the inclusion in the $\mathcal{M}_{90 \%}^{*}$ and $\mathcal{M}_{75 \%}^{*}$, respectively. The $t$-statistics for the parameter estimates are given in parentheses. 


\begin{tabular}{|c|c|}
\hline$h$ & 1 \\
\hline & \\
\hline adj. $R^{2}$ & 0.2262 \\
\hline $\begin{array}{l}\text { MSPE } \\
\text { MAPE }\end{array}$ & $\begin{array}{l}2.5390 \\
0.4220\end{array}$ \\
\hline QLIKE & $2.0430^{* *}$ \\
\hline & \\
\hline & \\
\hline & $\begin{array}{l}(6.0962) \\
0.6129\end{array}$ \\
\hline$R V^{(h)}$ & $\begin{array}{l}0.6129 \\
(8.5681)\end{array}$ \\
\hline adj. $R^{2}$ & 0.3753 \\
\hline MSPE & 2.2801 \\
\hline MAF & 0.5358 \\
\hline QLIKE & 2.4323 \\
\hline & \\
\hline & 0.0923 \\
\hline c & $(2.7729)$ \\
\hline$R V^{(h)}$ & 0.9714 \\
\hline & \\
\hline$\varepsilon^{(h)}$ & $\begin{array}{c}-0.7478 \\
(74.8161)\end{array}$ \\
\hline adj. $R^{2}$ & 0.4726 \\
\hline $\begin{array}{l}\text { MSPE } \\
\text { MAPE }\end{array}$ & $\begin{array}{c}2.0959^{*} \\
0.3989\end{array}$ \\
\hline & $\begin{array}{l}0.3989 \\
2.2463\end{array}$ \\
\hline & \\
\hline c & 0.2650 \\
\hline & $\begin{array}{c}(2.5424) \\
0.2170\end{array}$ \\
\hline$R V^{(1)}$ & $(2.4736)$ \\
\hline$R V^{(5)}$ & 0.3694 \\
\hline & $(3.6601)$ \\
\hline$R V^{(22)}$ & $\begin{array}{c}0.3318 \\
(4.1014)\end{array}$ \\
\hline adj. $R^{2}$ & 0.4843 \\
\hline MSPE & $2.0703^{*}$ \\
\hline MAPE & $0.3877^{* *}$ \\
\hline & \\
\hline & 0.192 \\
\hline c & (1.6696) \\
\hline$R V^{(1)}$ & 0.2009 \\
\hline & 0.3523 \\
\hline$R V^{(5)}$ & $(3.5286)$ \\
\hline$B V^{(22)}$ & 0.3099 \\
\hline$R V(22)$ & $(3.8850)$ \\
\hline$J^{(1)}$ & 0.4887 \\
\hline J1) & $(2.7537)$ \\
\hline adj. $\mathrm{R}^{2}$ & 0.4942 \\
\hline MSPE & $2.0498^{*}$ \\
\hline $\begin{array}{l}\text { MAPE } \\
\text { QLIKE }\end{array}$ & $\begin{array}{c}0.3871^{* *} \\
2.2162\end{array}$ \\
\hline & \\
\hline & 0.2060 \\
\hline & $(1.7492)$ \\
\hline$J^{(1)}$ & $\begin{array}{c}0.3086 \\
(2.8482)\end{array}$ \\
\hline$J^{(5)}$ & $\begin{array}{l}0.0063 \\
(0.1157)\end{array}$ \\
\hline$J^{(22)}$ & 0.0144 \\
\hline$J^{(22)}$ & $(0.7554)$ \\
\hline$C^{(1)}$ & 0.2396 \\
\hline & $(2.9244)$ \\
\hline$C^{(5)}$ & $\begin{array}{r}0.3184 \\
(3.4719)\end{array}$ \\
\hline$G^{(22)}$ & 0.2484 \\
\hline$C^{(22)}$ & (3.3097) \\
\hline adj. $R^{2}$ & 0.4962 \\
\hline MSPE & $2.0447^{*}$ \\
\hline MAPE & $0.3844^{* *}$ \\
\hline & \\
\hline & \\
\hline c & 0.2433 \\
\hline & $\begin{array}{l}(2.4254) \\
-0.1064\end{array}$ \\
\hline$R S^{+}$ & $\begin{array}{l}-0.1004 \\
(-0.6988)\end{array}$ \\
\hline$R S^{-}$ & 0.5064 \\
\hline$R S$ & $(2.4143)$ \\
\hline $\mathbf{I}_{r_{t}<0} R V^{(1)}$ & $\begin{array}{l}0.0048 \\
(0.0820)\end{array}$ \\
\hline & 0.3602 \\
\hline$R V^{(5)}$ & $(3.9271)$ \\
\hline$R V^{(22)}$ & $\begin{array}{l}0.3180 \\
\end{array}$ \\
\hline & \\
\hline $\begin{array}{l}\text { adj. } \mathrm{R}^{2} \\
\text { MSPE }\end{array}$ & $\begin{array}{c}0.5133 \\
2.0102^{*}\end{array}$ \\
\hline MAPE & 0.3943 \\
\hline QLIKE & 2.2139 \\
\hline & \\
\hline c & 0.1856 \\
\hline & \\
\hline$J^{(1)}$ & $\begin{array}{l}0.2166 \\
0(1.9988)\end{array}$ \\
\hline & $\begin{array}{l}(1.9988) \\
0.0099\end{array}$ \\
\hline$J^{(5)}$ & $(0.1992)$ \\
\hline$J^{(22)}$ & 0.0169 \\
\hline & $(0.9346)$ \\
\hline$C^{(1)}$ & 0.1594 \\
\hline & $(2.3619)$ \\
\hline$C^{(5)}$ & $\begin{array}{c}0.3479 \\
(3.9310)\end{array}$ \\
\hline$C^{(22)}$ & 0.2320 \\
\hline$C^{(22)}$ & $(3.1989)$ \\
\hline (1) & -0.4428 \\
\hline & $(-2.8705)$ \\
\hline & 0.0299 \\
\hline & $\begin{array}{l}(2.9978) \\
-0.2537\end{array}$ \\
\hline$r_{t}^{-,(2}$ & $\begin{array}{l}-0.2537 \\
(-0.8880)\end{array}$ \\
\hline adj. $R^{2}$ & 0.5257 \\
\hline MSPE & $1.9823^{* *}$ \\
\hline OUVE & 0.3964 \\
\hline QLIKE & 2.1898 \\
\hline
\end{tabular}

Table A.20: In-Sample regression results for Sugar with $M e d R V$. Note that ${ }^{*}$ and ${ }^{* *}$ indicate the inclusion in the $\mathcal{M}_{90 \%}^{*}$ and $\mathcal{M}_{75 \%}^{*}$, respectively. The $t$-statistics for the parameter estimates are given in parentheses. 


\begin{tabular}{|c|}
\hline$h$ \\
\hline \\
\hline adj. $R^{2}$ \\
\hline $\begin{array}{l}\text { adj. } \\
\text { MSPE }\end{array}$ \\
\hline MAPE \\
\hline \\
\hline \\
\hline $\begin{array}{l}\text { c } \\
R V^{(h)}\end{array}$ \\
\hline adj. $R^{2}$ \\
\hline MSPE \\
\hline MAPE \\
\hline QLIKE \\
\hline \\
\hline \\
\hline \\
\hline$R V^{(h)}$ \\
\hline \\
\hline$\varepsilon^{(h)}$ \\
\hline \\
\hline MSPE \\
\hline $\begin{array}{l}\text { MAPE } \\
\text { QLIKE }\end{array}$ \\
\hline \\
\hline c \\
\hline \\
\hline$R V^{(1)}$ \\
\hline$R V^{(5)}$ \\
\hline \\
\hline$R V^{(22)}$ \\
\hline adj. $R^{2}$ \\
\hline MSPE \\
\hline $\begin{array}{l}\text { MAPE } \\
\text { QLIKE }\end{array}$ \\
\hline \\
\hline \\
\hline c \\
\hline$R V^{(1)}$ \\
\hline \\
\hline$R V^{(5)}$ \\
\hline$R V^{(22)}$ \\
\hline$R V^{(22)}$ \\
\hline \\
\hline$J^{(1)}$ \\
\hline adj. $\mathrm{R}^{2}$ \\
\hline MSP \\
\hline MAPE \\
\hline QLIKE \\
\hline \\
\hline c \\
\hline \\
\hline$J^{(1)}$ \\
\hline$J^{(5)}$ \\
\hline \\
\hline$J^{(22)}$ \\
\hline \\
\hline$C^{(1)}$ \\
\hline \\
\hline$C^{(5)}$ \\
\hline$C^{(22)}$ \\
\hline$C^{(22)}$ \\
\hline adj. $\mathrm{R}^{2}$ \\
\hline $\begin{array}{l}\text { MSPE } \\
\text { MAPE }\end{array}$ \\
\hline QLIKE \\
\hline \\
\hline c \\
\hline \\
\hline$R S^{+}$ \\
\hline$R S^{-}$ \\
\hline \\
\hline $\mathbf{I}_{r_{t}<0} R V^{(1)}$ \\
\hline$B V^{(5)}$ \\
\hline$R V^{(5)}$ \\
\hline$R V^{(22)}$ \\
\hline \\
\hline adj. $\mathrm{R}^{2}$ \\
\hline MSPE \\
\hline MAPE \\
\hline QLIKE \\
\hline \\
\hline c \\
\hline \\
\hline$J^{(1)}$ \\
\hline \\
\hline$J^{(5)}$ \\
\hline$J^{(22)}$ \\
\hline \\
\hline$C^{(1}$ \\
\hline \\
\hline$C^{(5)}$ \\
\hline \\
\hline$C^{(22)}$ \\
\hline$r_{-,-(1)}^{-}$ \\
\hline \\
\hline$r_{t}$ \\
\hline \\
\hline$r_{t}^{-,(22)}$ \\
\hline $\mathrm{R}^{2}$ \\
\hline $\mathrm{PF}$ \\
\hline OLUKE \\
\hline QLIKE \\
\hline
\end{tabular}

Table A.21: In-Sample regression results for Wheat with $M e d R V$. Note that $*$ and $* *$ indicate the inclusion in the $\mathcal{M}_{90 \%}^{*}$ and $\mathcal{M}_{75 \%}^{*}$, respectively. The $t$-statistics for the parameter estimates are given in parentheses. 
Appendix A.2. Out-of-Sample

\begin{tabular}{|c|c|c|c|c|c|c|c|c|c|}
\hline & $h$ & RW & $\mathrm{AR}$ & ARMA & HAR & HAR-J & HAR-CJ & HAR-PS & LHAR-CJ \\
\hline \multirow{6}{*}{ MSPE } & 1 & 0.9890 & 0.8956 & 0.9527 & $0.8414^{* *}$ & $0.8420^{* *}$ & $0.8437^{* *}$ & $0.8479^{* *}$ & $0.8627^{*}$ \\
\hline & 5 & $1.0020^{*}$ & $0.9695^{* *}$ & 1.0371 & $0.9588^{* *}$ & $0.9580^{* *}$ & $0.9630^{* *}$ & $0.9601^{* *}$ & $0.9686^{* *}$ \\
\hline & 10 & $1.0453^{*}$ & $1.0148^{* *}$ & 1.0752 & $1.0207^{* *}$ & $1.0223^{* *}$ & $1.0294^{* *}$ & $1.0280^{*}$ & 1.0405 \\
\hline & 22 & 1.1325 & 1.0820 & 1.1263 & $1.0669^{* *}$ & $1.0684^{* *}$ & $1.0607^{* *}$ & $1.0678^{* *}$ & 1.0748 \\
\hline & 44 & 1.2243 & $1.1221^{* *}$ & $1.1184^{* *}$ & $1.1124^{* *}$ & $1.1122^{* *}$ & $1.0982^{* *}$ & $1.1089^{* *}$ & $1.1077^{* *}$ \\
\hline & 66 & 1.2886 & 1.1573 & 1.2960 & 1.1263 & 1.1283 & $1.1126^{* *}$ & 1.1260 & 1.1240 \\
\hline \multirow{6}{*}{ MAPE } & 1 & 0.5958 & 0.6332 & $0.5920^{* *}$ & $0.5810^{* *}$ & $0.5808^{* *}$ & $0.5790^{* *}$ & $0.5799^{* *}$ & 0.5920 \\
\hline & 5 & $0.6396^{* *}$ & 0.6650 & $0.6647^{*}$ & $0.6471^{* *}$ & $0.6468^{* *}$ & $0.6456^{* *}$ & $0.6458^{* *}$ & $0.6464^{* *}$ \\
\hline & 10 & $0.6451^{* *}$ & 0.6740 & 0.6829 & 0.6744 & 0.6763 & 0.6775 & 0.6756 & 0.6797 \\
\hline & 22 & $0.6834^{* *}$ & 0.7147 & 0.7430 & $0.7043^{*}$ & 0.7051 & $0.6973^{*}$ & $0.7026^{*}$ & 0.7055 \\
\hline & 44 & $0.7443^{* *}$ & 0.7644 & 0.7811 & 0.7506 & 0.7514 & $0.7366^{* *}$ & 0.7476 & 0.7455 \\
\hline & 66 & 0.7860 & 0.7945 & 0.8687 & 0.7696 & 0.7711 & $0.7563^{* *}$ & 0.7682 & 0.7666 \\
\hline \multirow{6}{*}{ QLIKE } & 1 & 1.5628 & 1.5390 & 1.5492 & $1.5277^{* *}$ & $1.5277^{* *}$ & $1.5280^{* *}$ & $1.5288^{* *}$ & $1.5300^{*}$ \\
\hline & 5 & 1.5664 & 1.5559 & 1.5702 & $1.5519^{* *}$ & $1.5516^{* *}$ & $1.5523^{* *}$ & $1.5515^{* *}$ & $1.5521^{* *}$ \\
\hline & 10 & $1.5709^{*}$ & $1.5636^{* *}$ & $1.5748^{*}$ & $1.5660^{* *}$ & $1.5662^{* *}$ & $1.5691^{*}$ & $1.5675^{*}$ & $1.5704^{*}$ \\
\hline & 22 & 1.5979 & $1.5828^{*}$ & 1.5954 & $1.5790^{* *}$ & $1.5792^{* *}$ & $1.5782^{* *}$ & $1.5792^{* *}$ & $1.5817^{*}$ \\
\hline & 44 & 1.6363 & $1.5976^{*}$ & $1.5865^{* *}$ & $1.5937^{* *}$ & $1.5935^{* *}$ & $1.5910^{* *}$ & $1.5928^{* *}$ & $1.5935^{* *}$ \\
\hline & 66 & 1.6662 & $1.5976^{*}$ & 1.6295 & 1.5970 & 1.5973 & $1.5935^{* *}$ & $1.5965^{*}$ & 1.5970 \\
\hline \multirow{6}{*}{ SR } & 1 & $0.7436^{* * *}$ & $0.7237^{* * *}$ & $0.7465^{* * *}$ & $0.7446^{* * *}$ & $0.7417^{* * *}$ & $0.7446^{* * *}$ & $0.7427^{* * *}$ & $0.7370^{* * *}$ \\
\hline & 5 & $0.6660^{* * *}$ & $0.6518^{* * *}$ & $0.6566^{* * *}$ & $0.6689^{* * *}$ & $0.6689^{* * *}$ & $0.6736^{* * *}$ & $0.6717^{* * *}$ & $0.6698^{* * *}$ \\
\hline & 10 & $0.6679^{* * *}$ & $0.6537^{* * *}$ & $0.6462^{* * *}$ & $0.6462^{* * *}$ & $0.6462^{* * *}$ & $0.6339^{* * *}$ & $0.6471^{* * *}$ & $0.6272^{* * *}$ \\
\hline & 22 & $0.6121^{* * *}$ & $0.5960^{* * *}$ & $0.5525^{* *}$ & $0.6216^{* * *}$ & $0.6235^{* * *}$ & $0.6301^{* * *}$ & $0.6244^{* * *}$ & $0.6083^{* * *}$ \\
\hline & 44 & 0.4768 & 0.4910 & $0.6026^{* * *}$ & 0.5412 & 0.5412 & $0.5572^{* * *}$ & $0.5468^{*}$ & 0.5241 \\
\hline & 66 & 0.4049 & 0.5449 & 0.4617 & 0.5203 & 0.5270 & 0.5374 & 0.5222 & 0.5071 \\
\hline
\end{tabular}

Table A.22: Forecasting Evaluation for Corn Futures with $M e d R V$. Note that ${ }^{*}$ and ${ }^{* *}$ indicate the inclusion in the $\mathcal{M}_{90 \%}^{*}$ and $\mathcal{M}_{75 \%}^{*}$, respectively. For the Success Ratio (SR), the asterisk *, ${ }^{* *}$, and ${ }^{* * *}$ indicate a statistical significance at $10 \%, 5 \%$, and $1 \%$, respectively.

\begin{tabular}{|c|c|c|c|c|c|c|c|c|c|}
\hline & $h$ & RW & $\mathrm{AR}$ & ARMA & HAR & HAR-J & HAR-CJ & HAR-PS & LHAR-CJ \\
\hline \multirow{6}{*}{ MSPE } & 1 & 1.7872 & 1.5022 & 1.7734 & $1.4042^{* *}$ & $1.4008^{* *}$ & $1.3951^{* *}$ & $1.3971^{* *}$ & $1.3987^{* *}$ \\
\hline & 5 & 1.5206 & 1.4697 & 1.5164 & $1.4366^{* *}$ & $1.4371^{* *}$ & $1.4319^{* *}$ & $1.4357^{* *}$ & $1.4320^{* *}$ \\
\hline & 10 & $1.4838^{*}$ & $1.4601^{* *}$ & 1.5317 & $1.4497^{* *}$ & $1.4521^{* *}$ & $1.4510^{* *}$ & $1.4505^{* *}$ & $1.4508^{* *}$ \\
\hline & 22 & $1.4654^{* *}$ & $1.4655^{* *}$ & $1.4404^{* *}$ & $1.4700^{* *}$ & $1.4675^{* *}$ & $1.4693^{* *}$ & $1.4677^{* *}$ & $1.4674^{* *}$ \\
\hline & 44 & $1.4340^{* *}$ & $1.4448^{* *}$ & 1.4916 & $1.4584^{* *}$ & $1.4570^{* *}$ & $1.4578^{* *}$ & $1.4560^{* *}$ & $1.4597^{* *}$ \\
\hline & 66 & $1.4682^{* *}$ & 1.4938 & 1.5501 & 1.5012 & 1.4988 & 1.5020 & 1.4989 & $1.4917^{*}$ \\
\hline \multirow{6}{*}{ MAPE } & 1 & $1.1032^{* *}$ & 1.2800 & $1.1056^{* *}$ & 1.1484 & $1.1368^{* *}$ & $1.1403^{* *}$ & $1.1340^{* *}$ & $1.1476^{* *}$ \\
\hline & 5 & 1.2344 & 1.2811 & $1.1979^{* *}$ & 1.2091 & $1.2045^{* *}$ & 1.2046 & $1.1893^{* *}$ & 1.2155 \\
\hline & 10 & $1.1429^{* *}$ & 1.2041 & 1.2064 & 1.1988 & 1.1977 & 1.2031 & 1.1979 & 1.2203 \\
\hline & 22 & $1.1650^{*}$ & 1.2438 & $1.1372^{* *}$ & 1.2448 & 1.2435 & 1.2528 & 1.2388 & 1.2836 \\
\hline & 44 & $1.1428^{* *}$ & 1.1838 & $1.1342^{* *}$ & 1.2380 & 1.2379 & 1.2366 & 1.2368 & 1.2709 \\
\hline & 66 & $1.1558^{* *}$ & 1.1803 & $1.1804^{*}$ & 1.2648 & 1.2641 & 1.2485 & 1.2632 & 1.3121 \\
\hline \multirow{6}{*}{ QLIKE } & 1 & 1.8923 & 1.3430 & 1.6712 & $1.2643^{*}$ & $1.2611^{* *}$ & $1.2567^{* *}$ & $1.2609^{* *}$ & $1.2571^{* *}$ \\
\hline & 5 & 1.3534 & 1.3250 & 1.3432 & 1.2931 & 1.2921 & $1.2851^{* *}$ & 1.2931 & $1.2847^{* *}$ \\
\hline & 10 & $1.3306^{*}$ & $1.3096^{*}$ & 1.3594 & $1.3001^{* *}$ & $1.3002^{* *}$ & $1.2959^{* *}$ & $1.3000^{* *}$ & $1.2932^{* *}$ \\
\hline & 22 & $1.3189^{* *}$ & $1.3169^{* *}$ & $1.3068^{* *}$ & $1.3200^{* *}$ & $1.3184^{* *}$ & $1.3140^{* *}$ & $1.3194^{* *}$ & $1.3074^{* *}$ \\
\hline & 44 & $1.2950^{* *}$ & $1.3037^{*}$ & 1.3722 & 1.3208 & 1.3209 & $1.3149^{*}$ & 1.3202 & $1.3086^{*}$ \\
\hline & 66 & $1.3301^{* *}$ & 1.3534 & 1.4147 & $1.3454^{*}$ & $1.3446^{*}$ & 1.3504 & $1.3441^{*}$ & $1.3262^{* *}$ \\
\hline \multirow{6}{*}{ SR } & 1 & $0.6907^{* * *}$ & $0.6129^{* * *}$ & $0.6879^{* * *}$ & $0.7068^{* * *}$ & $0.7125^{* * *}$ & $0.7059^{* * *}$ & $0.7125^{* * *}$ & $0.7135^{* * *}$ \\
\hline & 5 & $0.6898^{* * *}$ & $0.6433^{* * *}$ & $0.6803^{* * *}$ & $0.6784^{* * *}$ & $0.6765^{* * *}$ & $0.6736^{* * *}$ & $0.6755^{* * *}$ & $0.6784^{* * *}$ \\
\hline & 10 & $0.6717^{* * *}$ & $0.6565^{* * *}$ & $0.6499^{* * *}$ & $0.6537^{* * *}$ & $0.6556^{* * *}$ & $0.6556^{* * *}$ & $0.6594^{* * *}$ & $0.6670^{* * *}$ \\
\hline & 22 & $0.7040^{* * *}$ & $0.6347^{* * *}$ & $0.6879^{* * *}$ & $0.6376^{* * *}$ & $0.6433^{* * *}$ & $0.6357^{* * *}$ & $0.6490^{* * *}$ & $0.6546^{* * *}$ \\
\hline & 44 & $0.6869^{* * *}$ & $0.6784^{* * *}$ & $0.6822^{* * *}$ & $0.6319^{* * *}$ & $0.6395^{* * *}$ & $0.6518^{* * *}$ & $0.6461^{* * *}$ & $0.6698^{* * *}$ \\
\hline & 66 & $0.6793^{* * *}$ & $0.6879^{* * *}$ & $0.6452^{* * *}$ & $0.6129^{* * *}$ & $0.6186^{* * *}$ & $0.6471^{* * *}$ & $0.6243^{* * *}$ & $0.6641^{* * *}$ \\
\hline
\end{tabular}

Table A.23: Forecasting Evaluation for Rice Futures with $M e d R V$. Note that ${ }^{*}$ and ${ }^{* *}$ indicate the inclusion in the $\mathcal{M}_{90 \%}^{*}$ and $\mathcal{M}_{75 \%}^{*}$, respectively. For the Success Ratio (SR), the asterisk *, ${ }^{* *}$, and ${ }^{* * *}$ indicate a statistical significance at $10 \%, 5 \%$, and $1 \%$, respectively. 


\begin{tabular}{|c|c|c|c|c|c|c|c|c|c|}
\hline & $h$ & RW & $\mathrm{AR}$ & ARMA & HAR & HAR-J & HAR-CJ & HAR-PS & LHAR-CJ \\
\hline \multirow{6}{*}{ MSPE } & 1 & 0.8109 & 0.7237 & $0.7851^{*}$ & $0.6720^{* *}$ & $0.6725^{* *}$ & $0.6668^{* *}$ & $0.6715^{* *}$ & $0.6705^{* *}$ \\
\hline & 5 & 0.7649 & 0.7400 & 0.7888 & $0.7331^{* *}$ & $0.7333^{* *}$ & $0.7316^{* *}$ & 0.7376 & 0.7377 \\
\hline & 10 & 0.8182 & $0.7865^{* *}$ & 0.8119 & $0.7816^{* *}$ & $0.7821^{* *}$ & $0.7823^{* *}$ & $0.7822^{* *}$ & 0.7869 \\
\hline & 22 & 0.8929 & 0.8516 & 0.9107 & $0.8301^{* *}$ & $0.8311^{*}$ & $0.8265^{* *}$ & $0.8299^{* *}$ & 0.8354 \\
\hline & 44 & 0.9914 & 0.8973 & $0.8888^{* *}$ & $0.8840^{* *}$ & $0.8843^{* *}$ & 0.8899 & $0.8842^{* *}$ & 0.9009 \\
\hline & 66 & 1.0476 & $0.8969^{* *}$ & $0.9002^{* *}$ & $0.8998^{* *}$ & $0.9002^{* *}$ & $0.9049^{* *}$ & $0.9003^{* *}$ & 0.9212 \\
\hline \multirow{6}{*}{ MAPE } & 1 & 0.6117 & 0.6559 & 0.6081 & 0.5977 & 0.5982 & $0.5922^{* *}$ & $0.5961^{*}$ & 0.5984 \\
\hline & 5 & $0.6288^{* *}$ & 0.6624 & 0.6684 & 0.6511 & 0.6517 & 0.6494 & 0.6530 & 0.6583 \\
\hline & 10 & $0.6621^{* *}$ & 0.6968 & 0.7215 & 0.6891 & 0.6897 & 0.6868 & 0.6900 & 0.6912 \\
\hline & 22 & $0.7293^{* *}$ & 0.7618 & 0.8061 & $0.7441^{*}$ & $0.7446^{*}$ & $0.7396^{* *}$ & $0.7438^{*}$ & 0.7501 \\
\hline & 44 & $0.8140^{* *}$ & 0.8207 & 0.8422 & $0.8012^{* *}$ & $0.8014^{* *}$ & $0.8000^{* *}$ & $0.8011^{* *}$ & 0.8135 \\
\hline & 66 & 0.8676 & 0.8360 & $0.8194^{* *}$ & $0.8214^{* *}$ & $0.8213^{* *}$ & $0.8226^{* *}$ & $0.8209^{* *}$ & 0.8370 \\
\hline \multirow{6}{*}{ QLIKE } & 1 & 1.1557 & 1.1304 & 1.1413 & $1.1140^{* *}$ & $1.1142^{* *}$ & $1.1134^{* *}$ & $1.1139^{* *}$ & $1.1153^{*}$ \\
\hline & 5 & 1.1417 & 1.1362 & 1.1545 & $1.1330^{* *}$ & $1.1332^{* *}$ & $1.1327^{* *}$ & 1.1346 & 1.1350 \\
\hline & 10 & 1.1651 & $1.1545^{* *}$ & 1.1723 & $1.1548^{* *}$ & $1.1550^{* *}$ & $1.1562^{* *}$ & $1.1552^{* *}$ & 1.1584 \\
\hline & 22 & 1.2243 & 1.1897 & 1.2182 & $1.1791^{* *}$ & $1.1794^{* *}$ & $1.1791^{* *}$ & $1.1789^{* *}$ & 1.1828 \\
\hline & 44 & 1.2845 & 1.2156 & $1.2068^{* *}$ & $1.2077^{* *}$ & $1.2078^{* *}$ & 1.2118 & $1.2078^{* *}$ & 1.2173 \\
\hline & 66 & 1.3268 & $1.2234^{* *}$ & $1.2157^{* *}$ & $1.2179^{* *}$ & $1.2180^{* *}$ & $1.2195^{* *}$ & $1.2181^{* *}$ & 1.2297 \\
\hline \multirow{6}{*}{$\mathrm{SR}$} & 1 & $0.7881^{* * *}$ & $0.7806^{* * *}$ & $0.7872^{* * *}$ & $0.7957^{* * *}$ & $0.7947^{* * *}$ & $0.7853^{* * *}$ & $0.7947^{* * *}$ & $0.7947^{* * *}$ \\
\hline & 5 & $0.7542^{* * *}$ & $0.7495^{* * *}$ & $0.7307^{* * *}$ & $0.7533^{* * *}$ & $0.7552^{* * *}$ & $0.7486^{* * *}$ & $0.7514^{* * *}$ & $0.7552^{* * *}$ \\
\hline & 10 & $0.7298^{* * *}$ & $0.7203^{* * *}$ & $0.6883^{* * *}$ & $0.7401^{* * *}$ & $0.7354^{* * *}$ & $0.7147^{* * *}$ & $0.7269^{* * *}$ & $0.7119^{* * *}$ \\
\hline & 22 & $0.6422^{* * *}$ & $0.6403^{* * *}$ & $0.5753^{* * *}$ & $0.6723^{* * *}$ & $0.6685^{* * *}$ & $0.6450^{* * *}$ & $0.6676^{* * *}$ & $0.6412^{* * *}$ \\
\hline & 44 & 0.5009 & 0.4849 & $0.5348^{*}$ & $0.5480^{* *}$ & $0.5480^{* *}$ & $0.5414^{*}$ & $0.5471^{* *}$ & 0.5311 \\
\hline & 66 & 0.4162 & 0.3889 & 0.4595 & 0.4576 & 0.4595 & 0.4595 & 0.4557 & 0.4407 \\
\hline
\end{tabular}

Table A.24: Forecasting Evaluation for Soy Futures with $M e d R V$. Note that ${ }^{*}$ and ${ }^{* *}$ indicate the inclusion in the $\mathcal{M}_{90 \%}^{*}$ and $\mathcal{M}_{75 \%}^{*}$, respectively. For the Success Ratio (SR), the asterisk *, **, and *** indicate a statistical significance at $10 \%, 5 \%$, and $1 \%$, respectively.

\begin{tabular}{|c|c|c|c|c|c|c|c|c|c|}
\hline & $h$ & RW & $\mathrm{AR}$ & ARMA & HAR & HAR-J & HAR-CJ & HAR-PS & LHAR-CJ \\
\hline \multirow{6}{*}{ MSPE } & 1 & 1.3511 & 1.2559 & 1.3330 & $1.0899^{* *}$ & $1.0989^{* *}$ & $1.0898^{* *}$ & $1.0916^{* *}$ & 1.1039 \\
\hline & 5 & 1.2470 & 1.2311 & 1.2525 & $1.1789^{* *}$ & $1.1812^{*}$ & $1.1810^{* *}$ & $1.1842^{*}$ & $1.1862^{*}$ \\
\hline & 10 & $1.2169^{* *}$ & $1.2308^{* *}$ & 1.2933 & $1.2125^{* *}$ & $1.2131^{* *}$ & $1.2173^{* *}$ & $1.2134^{* *}$ & $1.2210^{* *}$ \\
\hline & 22 & $1.2859^{* *}$ & $1.2813^{* *}$ & $1.3286^{* *}$ & $1.2850^{* *}$ & $1.2859^{* *}$ & $1.2949^{* *}$ & $1.2869^{* *}$ & $1.2974^{* *}$ \\
\hline & 44 & $1.3138^{* *}$ & $1.3412^{* *}$ & $1.3569^{* *}$ & $1.3317^{* *}$ & $1.3318^{* *}$ & $1.3415^{* *}$ & $1.3321^{* *}$ & $1.3413^{* *}$ \\
\hline & 66 & $1.3051^{* *}$ & 1.3783 & 1.3771 & 1.4049 & 1.4046 & 1.3998 & 1.4049 & 1.3830 \\
\hline \multirow{6}{*}{ MAPE } & 1 & 0.6343 & 0.7299 & 0.6368 & 0.6109 & $0.6001^{* *}$ & $0.5985^{* *}$ & $0.6033^{* *}$ & $0.6103^{*}$ \\
\hline & 5 & $0.6452^{* *}$ & 0.6971 & $0.6473^{* *}$ & $0.6539^{*}$ & $0.6515^{* *}$ & $0.6489^{* *}$ & $0.6507^{* *}$ & $0.6465^{* *}$ \\
\hline & 10 & $0.6442^{* *}$ & 0.7095 & $0.6666^{*}$ & 0.6748 & 0.6733 & 0.6691 & 0.6722 & 0.6698 \\
\hline & 22 & $0.6793^{* *}$ & $0.7000^{*}$ & $0.6962 *$ & $0.6965^{*}$ & $0.6956^{*}$ & $0.6953^{*}$ & $0.6950^{*}$ & $0.7029^{*}$ \\
\hline & 44 & $0.7029^{* *}$ & $0.7003^{* *}$ & $0.7047^{* *}$ & 0.7218 & 0.7217 & 0.7297 & 0.7197 & 0.7338 \\
\hline & 66 & $0.6915^{* *}$ & 0.7091 & $0.7082^{*}$ & 0.7471 & 0.7480 & 0.7517 & 0.7464 & 0.7473 \\
\hline \multirow{6}{*}{ QLIKE } & 1 & 1.8012 & 1.8068 & 1.7944 & $1.7603^{* *}$ & $1.7602^{* *}$ & $1.7597^{* *}$ & $1.7608^{* *}$ & 1.9152 \\
\hline & 5 & $1.7956^{*}$ & 1.7961 & $1.7904^{*}$ & $1.7820^{* *}$ & $1.7825^{* *}$ & $1.7810^{* *}$ & $1.7826^{* *}$ & $1.7912^{* *}$ \\
\hline & 10 & $1.7877^{* *}$ & $1.7973^{* *}$ & 1.8131 & $1.7916^{* *}$ & $1.7917^{* *}$ & $1.7913^{* *}$ & $1.7917^{* *}$ & $1.8025^{*}$ \\
\hline & 22 & $1.8198^{* *}$ & $1.8198^{* *}$ & 1.8623 & $1.8182^{* *}$ & $1.8186^{* *}$ & $1.8191^{* *}$ & $1.8185^{* *}$ & $1.8246^{* *}$ \\
\hline & 44 & $1.8480^{* *}$ & $1.8518^{* *}$ & $1.8567^{* *}$ & $1.8526^{* *}$ & $1.8524^{* *}$ & $1.8474^{* *}$ & $1.8525^{* *}$ & $1.8495^{* *}$ \\
\hline & 66 & $1.8327^{* *}$ & 1.8595 & 1.8713 & 1.8714 & 1.8709 & 1.8596 & 1.8715 & 1.8601 \\
\hline \multirow{6}{*}{ SR } & 1 & $0.7502^{* * *}$ & $0.7352^{* * *}$ & $0.7474^{* * *}$ & $0.7728^{* * *}$ & $0.7690^{* * *}$ & $0.7596^{* * *}$ & $0.7634^{* * *}$ & $0.7559 * * *$ \\
\hline & 5 & $0.7324^{* * *}$ & $0.7192^{* * *}$ & $0.7362^{* * *}$ & $0.7352^{* * *}$ & $0.7333^{* * *}$ & $0.7333^{* * *}$ & $0.7352^{* * *}$ & $0.7305^{* * *}$ \\
\hline & 10 & $0.7315^{* * *}$ & $0.7296^{* * *}$ & $0.7023^{* * *}$ & $0.7324^{* * *}$ & $0.7324^{* * *}$ & $0.7268^{* * *}$ & $0.7408^{* * *}$ & $0.7305^{* * *}$ \\
\hline & 22 & $0.7023^{* * *}$ & $0.7155^{* * *}$ & $0.6977^{* * *}$ & $0.7183^{* * *}$ & $0.7155^{* * *}$ & $0.7014^{* * *}$ & $0.7183^{* * *}$ & $0.7042^{* * *}$ \\
\hline & 44 & $0.7080^{* * *}$ & $0.6967^{* * *}$ & $0.7258^{* * *}$ & $0.7005^{* * *}$ & $0.6948^{* * *}$ & $0.6751^{* * *}$ & $0.6958^{* * *}$ & $0.6779^{* * *}$ \\
\hline & 66 & $0.7502 * * *$ & $0.6770^{* * *}$ & $0.7042^{* * *}$ & $0.6545^{* * *}$ & $0.6563^{* * *}$ & $0.6460^{* * *}$ & $0.6563^{* * *}$ & $0.6657^{* * *}$ \\
\hline
\end{tabular}

Table A.25: Forecasting Evaluation for Sugar Futures with $M e d R V$. Note that * and ** indicate the inclusion in the $\mathcal{M}_{90 \%}^{*}$ and $\mathcal{M}_{75 \%}^{*}$, respectively. For the Success Ratio (SR), the asterisk *, ${ }^{* *}$, and ${ }^{* * *}$ indicate a statistical significance at $10 \%, 5 \%$, and $1 \%$, respectively. 


\begin{tabular}{|c|c|c|c|c|c|c|c|c|c|}
\hline & $h$ & RW & $A R$ & ARMA & HAR & HAR-J & HAR-CJ & HAR-PS & LHAR-CJ \\
\hline \multirow{6}{*}{ MSPE } & 1 & 1.2218 & 1.1210 & 1.1825 & $1.0307^{* *}$ & $1.0374^{* *}$ & $1.0375^{* *}$ & $1.0266^{* *}$ & $1.0458^{*}$ \\
\hline & 5 & 1.2676 & 1.2228 & 1.2854 & $1.1765^{* *}$ & $1.1779^{* *}$ & $1.1837^{* *}$ & $1.1734^{* *}$ & $1.1867^{* *}$ \\
\hline & 10 & $1.2522^{* *}$ & $1.2286^{* *}$ & $1.2633^{* *}$ & $1.2147^{* *}$ & $1.2149^{* *}$ & $1.2175^{* *}$ & $1.2165^{* *}$ & $1.2125^{* *}$ \\
\hline & 22 & $1.2550^{* *}$ & $1.2642^{* *}$ & $1.3113^{*}$ & $1.2626^{* *}$ & $1.2657^{* *}$ & $1.2682^{* *}$ & $1.2676^{* *}$ & $1.2699^{* *}$ \\
\hline & 44 & $1.3406^{* *}$ & $1.3420^{* *}$ & 1.4302 & $1.3330^{* *}$ & $1.3293^{* *}$ & $1.3231^{* *}$ & $1.3308^{* *}$ & $1.3239^{* *}$ \\
\hline & 66 & $1.3954^{* *}$ & $1.4170^{*}$ & 1.6375 & $1.3967^{* *}$ & $1.3997^{* *}$ & $1.3800^{* *}$ & $1.3989^{* *}$ & $1.3910^{* *}$ \\
\hline \multirow{6}{*}{ MAPE } & 1 & 0.6369 & 0.6978 & 0.6358 & $0.6224^{*}$ & 0.6277 & $0.6223^{*}$ & $0.6179^{* *}$ & 0.6402 \\
\hline & 5 & $0.6634^{* *}$ & 0.7119 & $0.6851^{* *}$ & $0.6774^{* *}$ & 0.6796 & $0.6759^{* *}$ & $0.6747^{* *}$ & 0.6930 \\
\hline & 10 & $0.6591^{* *}$ & 0.7088 & 0.6910 & 0.7007 & 0.7022 & 0.6981 & 0.7007 & 0.7150 \\
\hline & 22 & $0.6783^{* *}$ & 0.7316 & 0.7292 & 0.7284 & 0.7294 & 0.7250 & 0.7288 & 0.7458 \\
\hline & 44 & $0.7234^{* *}$ & 0.7634 & 0.8111 & 0.7713 & 0.7703 & 0.7669 & 0.7701 & 0.7905 \\
\hline & 66 & $0.7519^{* *}$ & 0.8104 & 0.8905 & 0.8074 & 0.8085 & 0.8050 & 0.8079 & 0.8320 \\
\hline \multirow{6}{*}{ QLIKE } & 1 & 1.7837 & 1.7570 & 1.7640 & $1.7348^{* *}$ & $1.7354^{* *}$ & $1.7351^{* *}$ & $1.7342^{* *}$ & 1.7384 \\
\hline & 5 & 1.7724 & 1.7693 & 1.7754 & $1.7584^{* *}$ & $1.7589^{* *}$ & $1.7590^{* *}$ & $1.7574^{* *}$ & $1.7608^{* *}$ \\
\hline & 10 & $1.7701^{* *}$ & $1.7710^{* *}$ & $1.7771^{* *}$ & $1.7689^{* *}$ & $1.7690^{* *}$ & $1.7687^{* *}$ & $1.7691^{* *}$ & $1.7683^{* *}$ \\
\hline & 22 & $1.7832^{* *}$ & $1.7846^{* *}$ & 1.8111 & $1.7828^{* *}$ & $1.7833^{* *}$ & $1.7834^{* *}$ & $1.7838^{* *}$ & $1.7821^{* *}$ \\
\hline & 44 & $1.8265^{* *}$ & $1.8164^{*}$ & 1.8283 & $1.8069^{* *}$ & $1.8060^{* *}$ & $1.8040^{* *}$ & $1.8064^{* *}$ & $1.7988^{* *}$ \\
\hline & 66 & $1.8418^{* *}$ & $1.8247^{* *}$ & 1.8784 & $1.8214^{* *}$ & $1.8220^{* *}$ & $1.8178^{* *}$ & $1.8220^{* *}$ & $1.8153^{* *}$ \\
\hline \multirow{6}{*}{ SR } & 1 & $0.7030^{* * *}$ & $0.6635^{* * *}$ & $0.7002^{* * *}$ & $0.7049^{* * *}$ & $0.7039^{* * *}$ & $0.7068^{* * *}$ & $0.7115^{* * *}$ & $0.7105^{* * *}$ \\
\hline & 5 & $0.6776^{* * *}$ & $0.6278^{* * *}$ & $0.6645^{* * *}$ & $0.6795^{* * *}$ & $0.6701^{* * *}$ & $0.6776^{* * *}$ & $0.6776^{* * *}$ & $0.6776^{* * *}$ \\
\hline & 10 & $0.6692^{* * *}$ & $0.6118^{* * *}$ & $0.6382^{* * *}$ & $0.6410^{* * *}$ & $0.6466^{* * *}$ & $0.6400^{* * *}$ & $0.6429^{* * *}$ & $0.6598^{* * *}$ \\
\hline & 22 & $0.6560^{* * *}$ & $0.5977^{* * *}$ & $0.5686^{* * *}$ & $0.6071^{* * *}$ & $0.6081^{* * *}$ & $0.6071^{* * *}$ & $0.6081^{* * *}$ & $0.6241^{* * *}$ \\
\hline & 44 & $0.5987^{* * *}$ & 0.5028 & 0.4737 & $0.5244^{* *}$ & $0.5291^{* *}$ & $0.5320^{* *}$ & $0.5226^{* *}$ & $0.5479^{* * *}$ \\
\hline & 66 & $0.5451^{* * *}$ & 0.4314 & 0.4173 & 0.4549 & 0.4549 & 0.4436 & 0.4455 & 0.4746 \\
\hline
\end{tabular}

Table A.26: Forecasting Evaluation for Wheat Futures with $M e d R V$. Note that ${ }^{*}$ and ${ }^{* *}$ indicate the inclusion in the $\mathcal{M}_{90 \%}^{*}$ and $\mathcal{M}_{75 \%}^{*}$, respectively. For the Success Ratio (SR), the asterisk ${ }^{*}$, ${ }^{* *}$, and ${ }^{* * *}$ indicate a statistical significance at $10 \%, 5 \%$, and $1 \%$, respectively. 


\section{Appendix B. Additional Out-of-Sample Results}

\begin{tabular}{|c|c|c|c|c|c|c|c|c|c|}
\hline & $h$ & RW & $\mathrm{AR}$ & ARMA & HAR & HAR-J & HAR-CJ & HAR-PS & LHAR-CJ \\
\hline \multirow{6}{*}{ MSPE } & 1 & 1.3038 & 1.1508 & 1.2400 & $1.0898^{* *}$ & $1.0901^{* *}$ & $1.0890^{* *}$ & $1.0992 *$ & $1.0993^{* *}$ \\
\hline & 5 & $0.7970^{* *}$ & $0.7839^{*}$ & 0.9020 & $0.7629^{* *}$ & $0.7638^{* *}$ & $0.7606^{* *}$ & $0.7680^{* *}$ & $0.7580^{* *}$ \\
\hline & 10 & $0.7694^{*}$ & 0.7501 & 0.8402 & $0.7252^{*}$ & $0.7246^{*}$ & $0.7164^{* *}$ & $0.7325^{*}$ & $0.7122^{* *}$ \\
\hline & 22 & $0.7619^{*}$ & 0.7307 & 0.8581 & $0.6849^{* *}$ & $0.6841^{* *}$ & $0.6824^{* *}$ & $0.6883^{* *}$ & $0.6849^{* *}$ \\
\hline & 44 & 0.8185 & 0.7428 & 0.7154 & $0.6786^{* *}$ & $0.6785^{* *}$ & $0.6762^{* *}$ & $0.6796^{* *}$ & 0.6943 \\
\hline & 66 & 0.8722 & 0.7726 & 0.7752 & $0.6547^{* *}$ & $0.6546^{* *}$ & $0.6471^{* *}$ & $0.6550^{* *}$ & 0.6642 \\
\hline \multirow{6}{*}{ MAPE } & 1 & 0.6093 & 0.5899 & 0.5988 & $0.5516^{* *}$ & $0.5515^{* *}$ & $0.5509^{* *}$ & 0.5550 & $0.5570^{*}$ \\
\hline & 5 & 0.5090 & 0.5008 & 0.5307 & $0.4809^{* *}$ & $0.4808^{* *}$ & $0.4778^{* *}$ & $0.4816^{* *}$ & $0.4782^{* *}$ \\
\hline & 10 & 0.4858 & 0.4695 & 0.4877 & $0.4592^{*}$ & $0.4593^{*}$ & $0.4544^{* *}$ & $0.4604^{*}$ & $0.4555^{* *}$ \\
\hline & 22 & 0.4697 & 0.4567 & 0.5277 & $0.4445^{*}$ & $0.4451^{*}$ & $0.4410^{* *}$ & $0.4449^{*}$ & $0.4454^{*}$ \\
\hline & 44 & 0.5347 & 0.5066 & 0.5215 & $0.4714^{* *}$ & 0.4723 & 0.4753 & 0.4722 & 0.4876 \\
\hline & 66 & 0.5778 & 0.5411 & 0.5547 & $0.4816^{* *}$ & $0.4821^{* *}$ & $0.4783^{* *}$ & $0.4822^{* *}$ & 0.4926 \\
\hline \multirow{6}{*}{ QLIKE } & 1 & 1.6887 & 1.6600 & 1.6798 & $1.6517^{* *}$ & $1.6519^{* *}$ & $1.6520^{* *}$ & 1.6535 & $1.6529^{* *}$ \\
\hline & 5 & 1.6777 & $1.6686^{* *}$ & 1.6962 & $1.6653^{* *}$ & $1.6654^{* *}$ & $1.6656^{* *}$ & $1.6656^{* *}$ & $1.6662^{* *}$ \\
\hline & 10 & 1.6894 & $1.6812^{*}$ & 1.6967 & $1.6778^{* *}$ & $1.6777^{* *}$ & $1.6769^{* *}$ & $1.6783^{* *}$ & $1.6774^{* *}$ \\
\hline & 22 & 1.7045 & 1.6967 & 1.7232 & $1.6902^{* *}$ & $1.6901^{* *}$ & $1.6902^{* *}$ & $1.6907^{* *}$ & $1.6914^{* *}$ \\
\hline & 44 & 1.7444 & 1.7214 & $1.7121^{* *}$ & $1.7094^{* *}$ & $1.7093^{* *}$ & $1.7084^{* *}$ & $1.7096^{* *}$ & 1.7119 \\
\hline & 66 & 1.7787 & 1.7339 & 1.7300 & $1.7197^{* *}$ & $1.7195^{* *}$ & $1.7177^{* *}$ & $1.7197^{* *}$ & 1.7208 \\
\hline \multirow{6}{*}{ SR } & 1 & $0.7606^{* * *}$ & $0.7389^{* * *}$ & $0.7597^{* * *}$ & $0.7597^{* * *}$ & $0.7578^{* * *}$ & $0.7635^{* * *}$ & $0.7540^{* * *}$ & $0.7588^{* * *}$ \\
\hline & 5 & $0.7313^{* * *}$ & $0.7275^{* * *}$ & $0.6850^{* * *}$ & $0.7408^{* * *}$ & $0.7389^{* * *}$ & $0.7351^{* * *}$ & $0.7398^{* * *}$ & $0.7294^{* * *}$ \\
\hline & 10 & $0.7275^{* * *}$ & $0.7417^{* * *}$ & $0.7502^{* * *}$ & $0.7588^{* * *}$ & $0.7597^{* * *}$ & $0.7550^{* * *}$ & $0.7625^{* * *}$ & $0.7550^{* * *}$ \\
\hline & 22 & $0.7351^{* * *}$ & $0.7342^{* * *}$ & $0.5695^{* * *}$ & $0.7597^{* * *}$ & $0.7559^{* * *}$ & $0.7521^{* * *}$ & $0.7578^{* * *}$ & $0.7446^{* * *}$ \\
\hline & 44 & $0.5601^{* * *}$ & $0.5440^{* * *}$ & $0.5412^{* * *}$ & $0.6878^{* * *}$ & $0.6840^{* * *}$ & $0.6698^{* * *}$ & $0.6916^{* * *}$ & $0.6291^{* * *}$ \\
\hline & 66 & 0.4295 & $0.5629^{* * *}$ & $0.5317^{* *}$ & $0.6339^{* * *}$ & $0.6216^{* * *}$ & $0.6254^{* * *}$ & $0.6310^{* * *}$ & $0.5781^{* * *}$ \\
\hline
\end{tabular}

Table B.27: Forecasting Evaluation for Corn Futures with average $R V_{t+h}^{(h)}$. Note that * and ** indicate the inclusion in the $\mathcal{M}_{90 \%}^{*}$ and $\mathcal{M}_{75 \%}^{*}$, respectively. For the Success Ratio (SR), the asterisk ${ }^{*},{ }^{* *}$, and $* * *$ indicate a statistical significance at $10 \%, 5 \%$, and $1 \%$, respectively.

\begin{tabular}{|c|c|c|c|c|c|c|c|c|c|}
\hline & $h$ & RW & $\mathrm{AR}$ & ARMA & HAR & HAR-J & HAR-CJ & HAR-PS & LHAR-CJ \\
\hline \multirow{6}{*}{ MSPE } & 1 & 2.4100 & 2.1404 & 2.3635 & $1.9934^{* *}$ & $1.9968^{* *}$ & $1.9967^{* *}$ & $1.9943^{* *}$ & $1.9838^{* *}$ \\
\hline & 5 & $1.3149^{* *}$ & 1.3366 & 1.3595 & $1.2690^{* *}$ & $1.2714^{* *}$ & $1.2791^{* *}$ & $1.2710^{* *}$ & $1.2486^{* *}$ \\
\hline & 10 & $1.0925^{* *}$ & $1.1439^{*}$ & 1.2533 & $1.1146^{* *}$ & $1.1180^{* *}$ & $1.1284^{* *}$ & $1.1168^{* *}$ & $1.0867^{* *}$ \\
\hline & 22 & $0.9978^{* *}$ & 1.0516 & $1.0265^{* *}$ & $1.0368^{*}$ & 1.0392 & 1.0564 & $1.0379^{*}$ & $0.9993^{* *}$ \\
\hline & 44 & $0.8255^{* *}$ & 0.8944 & 1.0277 & 0.9593 & 0.9616 & 0.9922 & 0.9598 & 0.9241 \\
\hline & 66 & $0.8404^{* *}$ & 0.9563 & 1.1987 & 0.9673 & 0.9706 & 1.0023 & 0.9676 & 0.9292 \\
\hline \multirow{6}{*}{ MAPE } & 1 & 0.8550 & 0.7907 & 0.8478 & $0.7343^{* *}$ & $0.7327^{* *}$ & $0.7354^{* *}$ & $0.7359^{*}$ & $0.7355^{* *}$ \\
\hline & 5 & 0.5819 & 0.5692 & 0.5996 & $0.5521^{* *}$ & $0.5518^{* *}$ & $0.5577^{*}$ & $0.5519^{* *}$ & $0.5549^{* *}$ \\
\hline & 10 & $0.5350^{* *}$ & $0.5331^{* *}$ & 0.5662 & $0.5245^{* *}$ & $0.5256^{* *}$ & 0.5356 & $0.5249^{* *}$ & $0.5279^{* *}$ \\
\hline & 22 & $0.5086^{* *}$ & $0.5233^{*}$ & 0.5598 & $0.5185^{* *}$ & $0.5193^{* *}$ & 0.5285 & $0.5189^{* *}$ & $0.5220^{* *}$ \\
\hline & 44 & $0.4980^{* *}$ & 0.5183 & 0.5574 & 0.5251 & 0.5259 & 0.5379 & 0.5254 & 0.5190 \\
\hline & 66 & $0.5050^{* *}$ & 0.5507 & 0.6087 & 0.5306 & 0.5307 & 0.5398 & 0.5308 & $0.5179^{*}$ \\
\hline \multirow{6}{*}{ QLIKE } & 1 & 2.0261 & 1.9471 & 2.0171 & $1.8804^{* *}$ & $1.8811^{* *}$ & $1.8829^{* *}$ & $1.8807^{* *}$ & $1.8781^{* *}$ \\
\hline & 5 & 1.9173 & 1.9147 & 1.9352 & $1.8983^{* *}$ & $1.8988^{* *}$ & $1.9014^{* *}$ & $1.8990^{* *}$ & $1.8953^{* *}$ \\
\hline & 10 & $1.9156^{*}$ & 1.9163 & 1.9574 & $1.9087^{*}$ & $1.9095^{*}$ & $1.9118^{*}$ & $1.9093^{*}$ & $1.9017^{* *}$ \\
\hline & 22 & $1.9237^{*}$ & 1.9260 & 1.9456 & $1.9230^{*}$ & 1.9239 & 1.9269 & $1.9234^{*}$ & $1.9121^{* *}$ \\
\hline & 44 & $1.9223^{* *}$ & 1.9329 & 1.9851 & 1.9319 & 1.9330 & 1.9382 & 1.9321 & $1.9203^{* *}$ \\
\hline & 66 & $1.9383^{*}$ & 1.9743 & 2.0416 & 1.9459 & 1.9473 & 1.9525 & 1.9460 & $1.9281^{* *}$ \\
\hline \multirow{6}{*}{ SR } & 1 & $0.7011^{* * *}$ & $0.6556^{* * *}$ & $0.7030^{* * *}$ & $0.7353^{* * *}$ & $0.7268^{* * *}$ & $0.7353^{* * *}$ & $0.7315^{* * *}$ & $0.7334^{* * *}$ \\
\hline & 5 & $0.7875^{* * *}$ & $0.7562^{* * *}$ & $0.7581^{* * *}$ & $0.7932^{* * *}$ & $0.7922^{* * *}$ & $0.7960^{* * *}$ & $0.7922^{* * *}$ & $0.7941^{* * *}$ \\
\hline & 10 & $0.8121^{* * *}$ & $0.7913^{* * *}$ & $0.7770^{* * *}$ & $0.8008^{* * *}$ & $0.8027^{* * *}$ & $0.8083^{* * *}$ & $0.8036^{* * *}$ & $0.8065^{* * *}$ \\
\hline & 22 & $0.8387^{* * *}$ & $0.7913^{* * *}$ & $0.7998^{* * *}$ & $0.7913^{* * *}$ & $0.7913^{* * *}$ & $0.7998^{* * *}$ & $0.7884^{* * *}$ & $0.8121^{* * *}$ \\
\hline & 44 & $0.8681^{* * *}$ & $0.8406^{* * *}$ & $0.8454^{* * *}$ & $0.8008^{* * *}$ & $0.8027^{* * *}$ & $0.8093^{* * *}$ & $0.8036^{* * *}$ & $0.8207^{* * *}$ \\
\hline & 66 & $0.8548^{* * *}$ & $0.7884^{* * *}$ & $0.7751^{* * *}$ & $0.7875^{* * *}$ & $0.7875^{* * *}$ & $0.7979^{* * *}$ & $0.7894^{* * *}$ & $0.7913^{* * *}$ \\
\hline
\end{tabular}

Table B.28: Forecasting Evaluation for Rice Futures with average $R V_{t+h}^{(h)}$. Note that * and ** indicate the inclusion in the $\mathcal{M}_{90 \%}^{*}$ and $\mathcal{M}_{75 \%}^{*}$, respectively. For the Success Ratio (SR), the asterisk ${ }^{*}$, **, and $* * *$ indicate a statistical significance at $10 \%, 5 \%$, and $1 \%$, respectively. 


\begin{tabular}{|c|c|c|c|c|c|c|c|c|c|}
\hline & $h$ & RW & $\mathrm{AR}$ & ARMA & HAR & HAR-J & HAR-CJ & HAR-PS & LHAR-CJ \\
\hline \multirow{6}{*}{ MSPE } & 1 & 0.9453 & 0.8394 & 0.9104 & $0.7848^{* *}$ & $0.7833^{* *}$ & $0.7841^{* *}$ & $0.7857^{* *}$ & $0.7843^{* *}$ \\
\hline & 5 & $0.5499^{*}$ & $0.5378^{*}$ & 0.5982 & $0.5281^{* *}$ & $0.5262^{* *}$ & $0.5280^{* *}$ & $0.5280^{* *}$ & $0.5319^{* *}$ \\
\hline & 10 & 0.5268 & 0.5202 & 0.5959 & $0.4986^{*}$ & $0.4962^{* *}$ & $0.5039^{*}$ & $0.4989^{*}$ & 0.5116 \\
\hline & 22 & 0.5610 & 0.5616 & 0.6847 & $0.5125^{* *}$ & $0.5122^{* *}$ & 0.5214 & $0.5129^{* *}$ & 0.5344 \\
\hline & 44 & 0.6527 & 0.6265 & 0.6115 & $0.5528^{* *}$ & $0.5529^{* *}$ & 0.5618 & $0.5527^{* *}$ & 0.5752 \\
\hline & 66 & 0.6976 & 0.6006 & $0.5689^{* *}$ & $0.5492^{* *}$ & $0.5496^{* *}$ & $0.5548^{* *}$ & $0.5494^{* *}$ & 0.5740 \\
\hline \multirow{6}{*}{ MAPE } & 1 & 0.6157 & 0.6015 & 0.6076 & $0.5549^{*}$ & $0.5531^{* *}$ & $0.5554^{*}$ & $0.5550^{*}$ & $0.5581^{*}$ \\
\hline & 5 & 0.4914 & 0.4803 & 0.5087 & $0.4725^{* *}$ & $0.4713^{* *}$ & $0.4742 *$ & $0.4726^{* *}$ & 0.4771 \\
\hline & 10 & $0.4801^{* *}$ & $0.4651^{* *}$ & 0.5007 & $0.4639^{* *}$ & $0.4633^{* *}$ & $0.4668^{* *}$ & $0.4642^{* *}$ & $0.4684^{* *}$ \\
\hline & 22 & 0.4911 & 0.4903 & 0.5765 & $0.4650^{* *}$ & $0.4655^{* *}$ & 0.4701 & 0.4664 & 0.4768 \\
\hline & 44 & 0.5549 & 0.5490 & 0.5650 & $0.5069^{* *}$ & $0.5073^{* *}$ & $0.5097^{* *}$ & $0.5071^{* *}$ & 0.5177 \\
\hline & 66 & 0.5905 & 0.5476 & 0.5400 & $0.5073^{* *}$ & $0.5076^{* *}$ & $0.5041^{* *}$ & $0.5076^{* *}$ & 0.5176 \\
\hline \multirow{6}{*}{ QLIKE } & 1 & 1.3287 & 1.2983 & 1.3209 & $1.2841^{* *}$ & $1.2837^{* *}$ & $1.2834^{* *}$ & $1.2839^{* *}$ & $1.2834^{* *}$ \\
\hline & 5 & 1.3014 & $1.2939^{* *}$ & 1.3119 & $1.2924^{* *}$ & $1.2921^{* *}$ & $1.2925^{* *}$ & $1.2923^{* *}$ & $1.2932^{* *}$ \\
\hline & 10 & 1.3165 & $1.3082^{*}$ & 1.3302 & $1.3045^{* *}$ & $1.3042^{* *}$ & $1.3055^{*}$ & $1.3045^{* *}$ & 1.3074 \\
\hline & 22 & 1.3531 & 1.3401 & 1.3793 & $1.3271^{* *}$ & $1.3271^{* *}$ & 1.3287 & $1.3271^{* *}$ & 1.3323 \\
\hline & 44 & 1.4162 & 1.3846 & 1.3778 & $1.3603^{* *}$ & $1.3604^{* *}$ & $1.3623^{* *}$ & $1.3603^{* *}$ & 1.3669 \\
\hline & 66 & 1.4569 & 1.4004 & $1.3901^{* *}$ & $1.3811^{* *}$ & $1.3812^{* *}$ & $1.3818^{* *}$ & $1.3811^{* *}$ & 1.3889 \\
\hline \multirow{6}{*}{ SR } & 1 & $0.7787^{* * *}$ & $0.7495^{* * *}$ & $0.7768^{* * *}$ & $0.7910^{* * *}$ & $0.7900^{* * *}$ & $0.7834^{* * *}$ & $0.7863^{* * *}$ & $0.7900^{* * *}$ \\
\hline & 5 & $0.8117^{* * *}$ & $0.8145^{* * *}$ & $0.7768^{* * *}$ & $0.8145^{* * *}$ & $0.8136^{* * *}$ & $0.8136^{* * *}$ & $0.8107^{* * *}$ & $0.8098^{* * *}$ \\
\hline & 10 & $0.8117^{* * *}$ & $0.7994^{* * *}$ & $0.7250^{* * *}$ & $0.8173^{* * *}$ & $0.8154^{* * *}$ & $0.8126^{* * *}$ & $0.8136^{* * *}$ & $0.7957^{* * *}$ \\
\hline & 22 & $0.7363^{* * *}$ & $0.6770^{* * *}$ & $0.5753^{* * *}$ & $0.7316^{* * *}$ & $0.7279^{* * *}$ & $0.7373^{* * *}$ & $0.7316^{* * *}$ & $0.7326^{* * *}$ \\
\hline & 44 & $0.6186^{* * *}$ & 0.4849 & 0.5104 & $0.6215^{* * *}$ & $0.6177^{* * *}$ & $0.6384^{* * *}$ & $0.6168^{* * *}$ & $0.6347^{* * *}$ \\
\hline & 66 & 0.4718 & 0.3484 & 0.4746 & $0.5254^{*}$ & 0.5226 & $0.5678^{* * *}$ & $0.5226^{*}$ & 0.5104 \\
\hline
\end{tabular}

Table B.29: Forecasting Evaluation for Soy Futures with average $R V_{t+h}^{(h)}$. Note that * and ** indicate the inclusion in the $\mathcal{M}_{90 \%}^{*}$ and $\mathcal{M}_{75 \%}^{*}$, respectively. For the Success Ratio (SR), the asterisk *, **, and *** indicate a statistical significance at $10 \%, 5 \%$, and $1 \%$, respectively.

\begin{tabular}{|c|c|c|c|c|c|c|c|c|c|}
\hline & $h \quad \mid$ & RW & $\mathrm{AR}$ & ARMA & HAR & HAR-J & HAR-CJ & HAR-PS & LHAR-CJ \\
\hline \multirow{6}{*}{ MSPE } & 1 & 1.6238 & 1.4626 & 1.5919 & $1.3174^{* *}$ & $1.3168^{* *}$ & $1.3167^{* *}$ & $1.3188^{* *}$ & $1.3190^{* *}$ \\
\hline & 5 & 0.9428 & 0.9402 & 1.0376 & $0.8756^{* *}$ & $0.8733^{* *}$ & $0.8722^{* *}$ & $0.8734^{* *}$ & $0.8797^{* *}$ \\
\hline & 10 & $0.8091^{* *}$ & $0.8237^{* *}$ & 0.9256 & $0.8039^{* *}$ & $0.8016^{* *}$ & $0.7969^{* *}$ & $0.8016^{* *}$ & $0.8048^{* *}$ \\
\hline & 22 & $0.7633^{* *}$ & 0.8125 & 0.9445 & $0.7857^{* *}$ & $0.7818^{* *}$ & $0.7768^{* *}$ & $0.7841^{* *}$ & $0.7877^{* *}$ \\
\hline & 44 & $0.7468^{* *}$ & 0.9151 & 0.9183 & 0.8476 & 0.8427 & 0.8356 & 0.8478 & 0.8646 \\
\hline & 66 & $0.7331^{* *}$ & 0.9530 & 0.8933 & 0.9321 & 0.9267 & 0.9092 & 0.9321 & 0.9240 \\
\hline \multirow{6}{*}{ MAPE } & 1 & 0.6537 & 0.6265 & 0.6480 & $0.5741^{* *}$ & $0.5748^{* *}$ & $0.5763^{*}$ & $0.5744^{* *}$ & $0.5784^{*}$ \\
\hline & 5 & 0.5125 & 0.5055 & 0.5425 & $0.4838^{* *}$ & $0.4839^{* *}$ & $0.4857^{* *}$ & $0.4840^{* *}$ & $0.4882^{* *}$ \\
\hline & 10 & $0.4810^{* *}$ & $0.4767^{* *}$ & 0.5185 & $0.4682^{* *}$ & $0.4688^{* *}$ & $0.4719^{* *}$ & $0.4675^{* *}$ & $0.4687^{* *}$ \\
\hline & 22 & $0.4867^{* *}$ & 0.4893 & 0.5345 & $0.4776^{* *}$ & $0.4772^{* *}$ & $0.4836^{* *}$ & $0.4764^{* *}$ & $0.4819^{* *}$ \\
\hline & 44 & $0.4757^{* *}$ & 0.5121 & 0.5278 & 0.5010 & 0.5000 & 0.5093 & 0.5003 & 0.5107 \\
\hline & 66 & $0.4635^{* *}$ & 0.5060 & 0.5241 & 0.5134 & 0.5122 & 0.5180 & 0.5131 & 0.5181 \\
\hline \multirow{6}{*}{ QLIKE } & 1 & 1.9622 & 1.9459 & 1.9591 & $1.9182^{* *}$ & $1.9179^{* *}$ & $1.9177^{* *}$ & $1.9189^{* *}$ & $1.9180^{* *}$ \\
\hline & 5 & 1.9441 & 1.9398 & 1.9603 & $1.9325^{* *}$ & $1.9321^{* *}$ & $1.9314^{* *}$ & $1.9321^{* *}$ & $1.9322^{* *}$ \\
\hline & 10 & $1.9439^{* *}$ & $1.9418^{* *}$ & 1.9672 & $1.9424^{* *}$ & $1.9420^{* *}$ & $1.9403^{* *}$ & $1.9421^{* *}$ & $1.9407^{* *}$ \\
\hline & 22 & $1.9586^{* *}$ & 1.9638 & 2.0159 & $1.9588^{*}$ & $1.9581^{*}$ & $1.9550^{* *}$ & $1.9587^{*}$ & $1.9543^{* *}$ \\
\hline & 44 & $1.9884^{* *}$ & 2.0186 & 2.0308 & 1.9908 & 1.9896 & $1.9826^{* *}$ & 1.9909 & $1.9830^{* *}$ \\
\hline & 66 & $1.9919^{* *}$ & 2.0413 & 2.0135 & 2.0190 & 2.0176 & $2.0062^{*}$ & 2.0193 & $2.0068^{*}$ \\
\hline \multirow{6}{*}{$\mathrm{SR}$} & 1 & $0.7333^{* * *}$ & $0.7352^{* * *}$ & $0.7362^{* * *}$ & $0.7662^{* * *}$ & $0.7624^{* * *}$ & $0.7681^{* * *}$ & $0.7624^{* * *}$ & $0.7718^{* * *}$ \\
\hline & 5 & $0.7822^{* * *}$ & $0.7784^{* * *}$ & $0.7793^{* * *}$ & $0.8038^{* * *}$ & $0.8103^{* * *}$ & $0.8047^{* * *}$ & $0.8150^{* * *}$ & $0.8103^{* * *}$ \\
\hline & 10 & $0.8282^{* * *}$ & $0.8319^{* * *}$ & $0.7953^{* * *}$ & $0.8291^{* * *}$ & $0.8300^{* * *}$ & $0.8169^{* * *}$ & $0.8300^{* * *}$ & $0.8169^{* * *}$ \\
\hline & 22 & $0.8188^{* * *}$ & $0.8244^{* * *}$ & $0.8028^{* * *}$ & $0.8357^{* * *}$ & $0.8254^{* * *}$ & $0.7962^{* * *}$ & $0.8376^{* * *}$ & $0.8254^{* * *}$ \\
\hline & 44 & $0.9296^{* * *}$ & $0.8451^{* * *}$ & $0.8648^{* * *}$ & $0.8761^{* * *}$ & $0.8601^{* * *}$ & $0.8441^{* * *}$ & $0.8667^{* * *}$ & $0.8648^{* * *}$ \\
\hline & 66 & $0.9408^{* * *}$ & $0.8141^{* * *}$ & $0.8197^{* * *}$ & $0.8225^{* * *}$ & $0.8216^{* * *}$ & $0.8225^{* * *}$ & $0.8310^{* * *}$ & $0.8319^{* * *}$ \\
\hline
\end{tabular}

Table B.30: Forecasting Evaluation for Sugar Futures with average $R V_{t+h}^{(h)}$. Note that * and ** indicate the inclusion in the $\mathcal{M}_{90 \%}^{*}$ and $\mathcal{M}_{75 \%}^{*}$, respectively. For the Success Ratio (SR), the asterisk ${ }^{*}$, **, and $* * *$ indicate a statistical significance at $10 \%, 5 \%$, and $1 \%$, respectively. 


\begin{tabular}{|c|c|c|c|c|c|c|c|c|c|}
\hline & $h$ & RW & $\mathrm{AR}$ & ARMA & HAR & HAR-J & HAR-CJ & HAR-PS & LHAR-CJ \\
\hline \multirow{6}{*}{ MSPE } & 1 & 1.4871 & 1.3285 & 1.4444 & $1.2476^{* *}$ & $1.2497^{* *}$ & $1.2575^{*}$ & $1.2418^{* *}$ & $1.2579^{*}$ \\
\hline & 5 & $0.9886^{*}$ & $0.9328^{*}$ & 1.1257 & $0.8978^{* *}$ & $0.8980^{* *}$ & $0.9099^{* *}$ & $0.8939^{* *}$ & $0.9055^{* *}$ \\
\hline & 10 & $0.8988^{*}$ & $0.8550^{* *}$ & 0.9496 & $0.8192^{* *}$ & $0.8198^{* *}$ & $0.8288^{* *}$ & $0.8182^{* *}$ & $0.8156^{* *}$ \\
\hline & 22 & $0.7592^{* *}$ & $0.7657^{*}$ & 0.9012 & $0.7411^{* *}$ & $0.7422^{* *}$ & $0.7546^{* *}$ & $0.7422^{* *}$ & $0.7393^{* *}$ \\
\hline & 44 & $0.7845^{* *}$ & 0.8259 & 0.9596 & $0.7539^{* *}$ & $0.7557^{* *}$ & $0.7676^{* *}$ & $0.7547^{* *}$ & $0.7531^{* *}$ \\
\hline & 66 & $0.8367^{* *}$ & 0.9112 & 1.1841 & $0.7922^{* *}$ & $0.7928^{* *}$ & $0.8024^{* *}$ & $0.7929^{* *}$ & $0.7813^{* *}$ \\
\hline \multirow{6}{*}{ MAPE } & 1 & 0.6271 & 0.6143 & 0.6204 & $0.5698^{* *}$ & 0.5721 & $0.5689^{* *}$ & $0.5682^{* *}$ & 0.5744 \\
\hline & 5 & 0.5057 & 0.4923 & 0.5262 & $0.4726^{* *}$ & $0.4729^{* *}$ & $0.4742^{* *}$ & $0.4735^{* *}$ & $0.4779^{* *}$ \\
\hline & 10 & $0.4655^{* *}$ & $0.4551^{* *}$ & 0.4824 & $0.4498^{* *}$ & $0.4503^{* *}$ & $0.4515^{* *}$ & $0.4504^{* *}$ & $0.4537^{* *}$ \\
\hline & 22 & $0.4521^{*}$ & 0.4504 & 0.5030 & $0.4409^{* *}$ & $0.4415^{*}$ & $0.4458^{*}$ & $0.4411^{* *}$ & $0.4459^{*}$ \\
\hline & 44 & $0.4675^{* *}$ & 0.4843 & 0.5609 & $0.4672^{* *}$ & $0.4676^{* *}$ & $0.4710^{* *}$ & $0.4676^{* *}$ & $0.4687^{* *}$ \\
\hline & 66 & $0.4927^{* *}$ & 0.5402 & 0.6319 & $0.4986^{* *}$ & $0.4983^{* *}$ & $0.5004^{*}$ & $0.4990^{*}$ & $0.5004^{* *}$ \\
\hline \multirow{6}{*}{ QLIKE } & 1 & 1.9467 & 1.9167 & 1.9393 & $1.9018^{* *}$ & $1.9018^{* *}$ & $1.9032 *$ & $1.9017^{* *}$ & $1.9029^{* *}$ \\
\hline & 5 & 1.9262 & 1.9188 & 1.9443 & $1.9134^{* *}$ & $1.9133^{* *}$ & 1.9153 & $1.9134^{* *}$ & $1.9139^{* *}$ \\
\hline & 10 & 1.9304 & $1.9252^{* *}$ & 1.9385 & $1.9222^{* *}$ & $1.9221^{* *}$ & $1.9244^{*}$ & $1.9222^{* *}$ & $1.9217^{* *}$ \\
\hline & 22 & 1.9358 & 1.9350 & 1.9697 & $1.9316^{* *}$ & $1.9317^{* *}$ & 1.9336 & $1.9318^{* *}$ & $1.9297^{* *}$ \\
\hline & 44 & 1.9672 & 1.9719 & 1.9942 & $1.9537^{*}$ & $1.9538^{*}$ & 1.9554 & $1.9538^{*}$ & $1.9493^{* *}$ \\
\hline & 66 & 1.9905 & 1.9934 & 2.0376 & $1.9705^{*}$ & $1.9703^{*}$ & 1.9708 & 1.9706 & $1.9648^{* *}$ \\
\hline \multirow{6}{*}{ SR } & 1 & $0.7124^{* * *}$ & $0.6805^{* * *}$ & $0.7143^{* * *}$ & $0.7105^{* * *}$ & $0.7077^{* * *}$ & $0.7049^{* * *}$ & $0.7180^{* * *}$ & $0.7068^{* * *}$ \\
\hline & 5 & $0.7011^{* * *}$ & $0.6842^{* * *}$ & $0.6814^{* * *}$ & $0.7359^{* * *}$ & $0.7312^{* * *}$ & $0.7246^{* * *}$ & $0.7368^{* * *}$ & $0.7284^{* * *}$ \\
\hline & 10 & $0.8083^{* * *}$ & $0.7735^{* * *}$ & $0.7556^{* * *}$ & $0.8045^{* * *}$ & $0.8083^{* * *}$ & $0.7989^{* * *}$ & $0.8036^{* * *}$ & $0.8017^{* * *}$ \\
\hline & 22 & $0.8336^{* * *}$ & $0.7679^{* * *}$ & $0.7068^{* * *}$ & $0.7876^{* * *}$ & $0.7857^{* * *}$ & $0.8139^{* * *}$ & $0.7838^{* * *}$ & $0.8299^{* * *}$ \\
\hline & 44 & $0.7368^{* * *}$ & $0.6241^{* * *}$ & 0.4154 & $0.6842^{* * *}$ & $0.6917^{* * *}$ & $0.7058^{* * *}$ & $0.6852^{* * *}$ & $0.7378^{* * *}$ \\
\hline & 66 & $0.6457^{* * *}$ & 0.4352 & 0.4126 & $0.5677^{* * *}$ & $0.5714^{* * *}$ & $0.5846^{* * *}$ & $0.5714^{* * *}$ & $0.6156^{* * *}$ \\
\hline
\end{tabular}

Table B.31: Forecasting Evaluation for Wheat Futures with average $R V_{t+h}^{(h)}$. Note that $*$ and $* *$ indicate the inclusion in the $\mathcal{M}_{90 \%}^{*}$ and $\mathcal{M}_{75 \%}^{*}$, respectively. For the Success Ratio (SR), the asterisk *, ${ }^{* *}$, and *** indicate a statistical significance at $10 \%, 5 \%$, and $1 \%$, respectively.

\begin{tabular}{|c|c|c|c|c|c|c|c|c|c|}
\hline & $h$ & RW & $\mathrm{AR}$ & ARMA & HAR & HAR-J & HAR-CJ & HAR-PS & LHAR-CJ \\
\hline \multirow{6}{*}{ MSPE } & 1 & 0.9890 & 0.8956 & 0.9527 & $0.8414^{* *}$ & $0.8420^{* *}$ & $0.8437^{* *}$ & $0.8479^{* *}$ & $0.8627^{* *}$ \\
\hline & 5 & 0.6689 & 0.6382 & 0.7405 & $0.6127^{* *}$ & $0.6114^{* *}$ & $0.6196^{* *}$ & $0.6127^{* *}$ & $0.6292^{*}$ \\
\hline & 10 & $0.6323^{*}$ & $0.6115^{*}$ & 0.7012 & $0.5904^{* *}$ & $0.5906^{* *}$ & $0.6008^{* *}$ & $0.5948^{* *}$ & 0.6139 \\
\hline & 22 & 0.6381 & 0.6140 & 0.7300 & $0.5721^{* *}$ & $0.5737^{* *}$ & $0.5767^{* *}$ & $0.5757^{* *}$ & 0.5924 \\
\hline & 44 & 0.6936 & 0.6521 & 0.7100 & $0.5903^{* *}$ & $0.5916^{* *}$ & $0.5790^{* *}$ & $0.5909^{* *}$ & 0.6061 \\
\hline & 66 & 0.7470 & 0.7475 & 0.8598 & 0.6111 & 0.6117 & $0.5895^{* *}$ & 0.6100 & 0.6062 \\
\hline \multirow{6}{*}{ MAPE } & 1 & 0.5958 & 0.6332 & $0.5920^{* *}$ & $0.5810^{* *}$ & $0.5808^{* *}$ & $0.5790^{* *}$ & $0.5799^{* *}$ & 0.5920 \\
\hline & 5 & $0.5204^{* *}$ & 0.5363 & 0.5498 & $0.5115^{* *}$ & $0.5112^{* *}$ & $0.5119^{* *}$ & $0.5101^{* *}$ & $0.5125^{* *}$ \\
\hline & 10 & $0.4905^{* *}$ & $0.5096^{*}$ & $0.5213^{*}$ & $0.4977^{* *}$ & $0.4980^{* *}$ & $0.5015^{* *}$ & $0.4970^{* *}$ & $0.5045^{* *}$ \\
\hline & 22 & $0.4827^{* *}$ & 0.5140 & 0.5766 & $0.4984^{* *}$ & $0.4992^{* *}$ & $0.4963^{* *}$ & $0.4983^{* *}$ & 0.5053 \\
\hline & 44 & $0.5486^{* *}$ & 0.5674 & 0.5955 & $0.5324^{* *}$ & $0.5327^{* *}$ & $0.5250^{* *}$ & $0.5316^{* *}$ & 0.5421 \\
\hline & 66 & 0.5882 & 0.6118 & 0.6610 & 0.5504 & 0.5509 & $0.5408^{* *}$ & 0.5490 & 0.5509 \\
\hline \multirow{6}{*}{ QLIKE } & 1 & 1.5628 & 1.5390 & 1.5492 & $1.5277^{* *}$ & $1.5277^{* *}$ & $1.5280^{* *}$ & $1.5288^{* *}$ & $1.5300^{*}$ \\
\hline & 5 & 1.5534 & 1.5466 & 1.5624 & $1.5415^{* *}$ & $1.5413^{* *}$ & $1.5423^{* *}$ & $1.5414^{* *}$ & $1.5426^{* *}$ \\
\hline & 10 & $1.5590^{*}$ & $1.5551^{*}$ & 1.5637 & $1.5519^{* *}$ & $1.5518^{* *}$ & $1.5534^{* *}$ & $1.5520 * *$ & $1.5541^{*}$ \\
\hline & 22 & 1.5712 & 1.5683 & 1.5881 & $1.5633^{* *}$ & $1.5634^{* *}$ & $1.5641^{* *}$ & $1.5636^{* *}$ & 1.5664 \\
\hline & 44 & 1.6037 & 1.5935 & 1.5954 & $1.5825^{* *}$ & $1.5825^{* *}$ & $1.5814^{* *}$ & $1.5825^{* *}$ & 1.5855 \\
\hline & 66 & 1.6304 & 1.6141 & 1.6252 & 1.5963 & 1.5963 & $1.5941^{* *}$ & 1.5961 & 1.5966 \\
\hline \multirow{6}{*}{$\mathrm{SR}$} & 1 & $0.7436^{* * *}$ & $0.7237^{* * *}$ & $0.7465^{* * *}$ & $0.7446^{* * *}$ & $0.7417^{* * *}$ & $0.7446^{* * *}$ & $0.7427^{* * *}$ & $0.7370^{* * *}$ \\
\hline & 5 & $0.7171^{* * *}$ & $0.7086^{* * *}$ & $0.6944^{* * *}$ & $0.7408^{* * *}$ & $0.7389^{* * *}$ & $0.7455^{* * *}$ & $0.7360^{* * *}$ & $0.7455^{* * *}$ \\
\hline & 10 & $0.7465^{* * *}$ & $0.7398^{* * *}$ & $0.7247^{* * *}$ & $0.7531^{* * *}$ & $0.7531^{* * *}$ & $0.7408^{* * *}$ & $0.7578^{* * *}$ & $0.7379^{* * *}$ \\
\hline & 22 & $0.7171^{* * *}$ & $0.7483^{* * *}$ & $0.6140^{* * *}$ & $0.7512^{* * *}$ & $0.7531^{* * *}$ & $0.7616^{* * *}$ & $0.7483^{* * *}$ & $0.7342^{* * *}$ \\
\hline & 44 & $0.5828^{* * *}$ & $0.5648^{* * *}$ & $0.5932^{* * *}$ & $0.6717^{* * *}$ & $0.6660^{* * *}$ & $0.6689^{* * *}$ & $0.6679^{* * *}$ & $0.6131^{* * *}$ \\
\hline & 66 & 0.4021 & $0.5553^{* * *}$ & 0.4286 & $0.5837^{* * *}$ & $0.5847^{* * *}$ & $0.5629^{* * *}$ & $0.5818^{* * *}$ & 0.5118 \\
\hline
\end{tabular}

Table B.32: Forecasting Evaluation for Corn Futures with average $M e d R V_{t+h}^{(h)}$. Note that * and ** indicate the inclusion in the $\mathcal{M}_{90 \%}^{*}$ and $\mathcal{M}_{75 \%}^{*}$, respectively. For the Success Ratio (SR), the asterisk *, **, and ${ }^{* * *}$ indicate a statistical significance at $10 \%, 5 \%$, and $1 \%$, respectively. 


\begin{tabular}{|c|c|c|c|c|c|c|c|c|c|}
\hline & $h$ & RW & $\mathrm{AR}$ & ARMA & HAR & HAR-J & HAR-CJ & HAR-PS & LHAR-CJ \\
\hline \multirow{6}{*}{ MSPE } & 1 & 1.7872 & 1.5022 & 1.7734 & $1.4042^{* *}$ & $1.4008^{* *}$ & $1.3951^{* *}$ & $1.3971^{* *}$ & $1.3987^{* *}$ \\
\hline & 5 & 0.9371 & 0.8589 & 0.9408 & $0.7933^{* *}$ & $0.7923^{* *}$ & $0.7850^{* *}$ & $0.7943^{* *}$ & $0.7868^{* *}$ \\
\hline & 10 & $0.6975^{* *}$ & $0.6660^{* *}$ & 0.8068 & $0.6641^{* *}$ & $0.6627^{* *}$ & $0.6574^{* *}$ & $0.6634^{* *}$ & $0.6574^{* *}$ \\
\hline & 22 & $0.6035^{* *}$ & $0.5986^{* *}$ & $0.5750^{* *}$ & $0.5976^{* *}$ & $0.5970^{* *}$ & $0.5998^{* *}$ & $0.5963^{* *}$ & $0.5970^{* *}$ \\
\hline & 44 & $0.4266^{* *}$ & 0.4451 & 0.5269 & 0.5139 & 0.5140 & 0.5133 & 0.5127 & 0.5107 \\
\hline & 66 & $0.4194^{* *}$ & 0.4707 & 0.6167 & 0.5193 & 0.5203 & 0.5089 & 0.5174 & 0.5115 \\
\hline \multirow{6}{*}{ MAPE } & 1 & $1.1032^{* *}$ & 1.2800 & $1.1056^{* *}$ & 1.1484 & $1.1368^{* *}$ & $1.1403^{* *}$ & $1.1340^{* *}$ & $1.1476^{* *}$ \\
\hline & 5 & 0.7461 & 0.7943 & 0.7539 & 0.7343 & $0.7304^{* *}$ & $0.7230^{* *}$ & $0.7301^{* *}$ & $0.7280^{* *}$ \\
\hline & 10 & $0.6414^{* *}$ & $0.6791^{*}$ & 0.6962 & $0.6677^{*}$ & $0.6655^{* *}$ & $0.6578^{* *}$ & $0.6651^{* *}$ & $0.6649^{* *}$ \\
\hline & 22 & $0.5871^{* *}$ & 0.6458 & $0.5982^{* *}$ & 0.6388 & 0.6379 & 0.6354 & 0.6361 & 0.6459 \\
\hline & 44 & $0.5208^{* *}$ & 0.5444 & 0.5647 & 0.6033 & 0.6030 & 0.5943 & 0.6015 & 0.5987 \\
\hline & 66 & $0.5096^{* *}$ & 0.5483 & 0.6212 & 0.6063 & 0.6054 & 0.5913 & 0.6036 & 0.5841 \\
\hline \multirow{6}{*}{ QLIKE } & 1 & 1.8923 & 1.3430 & 1.6712 & $1.2643^{*}$ & $1.2611^{* *}$ & $1.2567^{* *}$ & $1.2609^{* *}$ & $1.2571^{* *}$ \\
\hline & 5 & 1.3328 & 1.3152 & 1.3344 & 1.2826 & 1.2813 & $1.2748^{* *}$ & 1.2828 & $1.2746^{* *}$ \\
\hline & 10 & 1.3024 & 1.2952 & 1.3368 & 1.2905 & 1.2893 & $1.2835^{* *}$ & 1.2901 & $1.2804^{* *}$ \\
\hline & 22 & $1.3035^{* *}$ & $1.3063^{*}$ & $1.3125^{*}$ & $1.3048^{*}$ & $1.3041^{*}$ & $1.2993^{* *}$ & $1.3043^{*}$ & $1.2947^{* *}$ \\
\hline & 44 & $1.2919^{* *}$ & $1.2925^{* *}$ & 1.3237 & 1.3092 & 1.3090 & 1.3036 & 1.3086 & $1.2989^{* *}$ \\
\hline & 66 & $1.2975^{* *}$ & 1.3093 & 1.3593 & 1.3188 & 1.3189 & 1.3118 & 1.3181 & $1.3038^{* *}$ \\
\hline \multirow{6}{*}{ SR } & 1 & $0.6907^{* * *}$ & $0.6129^{* * *}$ & $0.6879^{* * *}$ & $0.7068^{* * *}$ & $0.7125^{* * *}$ & $0.7059^{* * *}$ & $0.7125^{* * *}$ & $0.7135^{* * *}$ \\
\hline & 5 & $0.7448^{* * *}$ & $0.6907^{* * *}$ & $0.7163^{* * *}$ & $0.7562^{* * *}$ & $0.7543^{* * *}$ & $0.7495^{* * *}$ & $0.7495^{* * *}$ & $0.7543^{* * *}$ \\
\hline & 10 & $0.7704^{* * *}$ & $0.7419^{* * *}$ & $0.7125^{* * *}$ & $0.7581^{* * *}$ & $0.7543^{* * *}$ & $0.7619^{* * *}$ & $0.7543^{* * *}$ & $0.7524^{* * *}$ \\
\hline & 22 & $0.8017^{* * *}$ & $0.7362^{* * *}$ & $0.8008^{* * *}$ & $0.7486^{* * *}$ & $0.7372^{* * *}$ & $0.7429^{* * *}$ & $0.7410^{* * *}$ & $0.7751^{* * *}$ \\
\hline & 44 & $0.8776^{* * *}$ & $0.8178^{* * *}$ & $0.8159^{* * *}$ & $0.7201^{* * *}$ & $0.7239^{* * *}$ & $0.7704^{* * *}$ & $0.7268^{* * *}$ & $0.8017^{* * *}$ \\
\hline & 66 & $0.8605^{* * *}$ & $0.7685^{* * *}$ & $0.7657^{* * *}$ & $0.6860^{* * *}$ & $0.6898^{* * *}$ & $0.7713^{* * *}$ & $0.6973^{* * *}$ & $0.8150^{* * *}$ \\
\hline
\end{tabular}

Table B.33: Forecasting Evaluation for Rice Futures with average $M e d R V_{t+h}^{(h)}$. Note that * and ** indicate the inclusion in the $\mathcal{M}_{90 \%}^{*}$ and $\mathcal{M}_{75 \%}^{*}$, respectively. For the Success Ratio (SR), the asterisk *, ${ }^{* *}$, and *** indicate a statistical significance at $10 \%, 5 \%$, and $1 \%$, respectively.

\begin{tabular}{|c|c|c|c|c|c|c|c|c|c|}
\hline & $h$ & RW & $\mathrm{AR}$ & ARMA & HAR & HAR-J & HAR-CJ & HAR-PS & LHAR-CJ \\
\hline \multirow{6}{*}{ MSPE } & 1 & 0.8109 & 0.7237 & $0.7851^{*}$ & $0.6720^{* *}$ & $0.6725^{* *}$ & $0.6668^{* *}$ & $0.6715^{* *}$ & $0.6705^{* *}$ \\
\hline & 5 & 0.5000 & 0.4720 & 0.5388 & $0.4594^{* *}$ & $0.4604^{*}$ & $0.4554^{* *}$ & $0.4611^{*}$ & 0.4624 \\
\hline & 10 & 0.4798 & 0.4562 & 0.5248 & $0.4348^{* *}$ & $0.4359^{*}$ & $0.4344^{* *}$ & $0.4371^{*}$ & 0.4412 \\
\hline & 22 & 0.4929 & 0.4757 & 0.6137 & $0.4395^{* *}$ & $0.4408^{*}$ & $0.4384^{* *}$ & $0.4405^{* *}$ & 0.4499 \\
\hline & 44 & 0.5774 & 0.5443 & 0.5439 & $0.4742^{* *}$ & $0.4751^{* *}$ & 0.4805 & $0.4745^{* *}$ & 0.4973 \\
\hline & 66 & 0.6207 & 0.5460 & 0.5333 & $0.4774^{* *}$ & $0.4780^{* *}$ & 0.4895 & $0.4772^{* *}$ & 0.5122 \\
\hline \multirow{6}{*}{ MAPE } & 1 & 0.6117 & 0.6559 & 0.6081 & 0.5977 & 0.5982 & $0.5922^{* *}$ & $0.5961^{*}$ & 0.5984 \\
\hline & 5 & $0.5058^{* *}$ & 0.5353 & 0.5477 & $0.5183^{* *}$ & $0.5193^{* *}$ & $0.5167^{* *}$ & $0.5185^{* *}$ & 0.5254 \\
\hline & 10 & $0.5000^{* *}$ & 0.5323 & 0.5654 & $0.5165^{* *}$ & $0.5171^{* *}$ & $0.5144^{* *}$ & $0.5175^{* *}$ & 0.5221 \\
\hline & 22 & $0.5283^{* *}$ & 0.5733 & 0.6557 & $0.5432^{* *}$ & $0.5438^{* *}$ & $0.5410^{* *}$ & $0.5428^{* *}$ & 0.5520 \\
\hline & 44 & $0.5923^{* *}$ & 0.6186 & 0.6397 & $0.5725^{* *}$ & $0.5727^{* *}$ & $0.5704^{* *}$ & $0.5720^{* *}$ & 0.5854 \\
\hline & 66 & 0.6354 & 0.6232 & 0.6027 & $0.5750^{* *}$ & $0.5748^{* *}$ & $0.5754^{* *}$ & $0.5742^{* *}$ & 0.5943 \\
\hline \multirow{6}{*}{ QLIKE } & 1 & 1.1557 & 1.1304 & 1.1413 & $1.1140^{* *}$ & $1.1142^{* *}$ & $1.1134^{* *}$ & $1.1139^{* *}$ & $1.1153^{*}$ \\
\hline & 5 & 1.1344 & 1.1303 & 1.1438 & $1.1264^{* *}$ & $1.1267^{* *}$ & $1.1262^{* *}$ & $1.1268^{* *}$ & 1.1279 \\
\hline & 10 & 1.1462 & 1.1434 & 1.1610 & $1.1391^{* *}$ & $1.1393^{* *}$ & $1.1393^{* *}$ & $1.1394^{* *}$ & 1.1411 \\
\hline & 22 & 1.1805 & 1.1720 & 1.2140 & $1.1623^{* *}$ & $1.1625^{* *}$ & $1.1628^{* *}$ & $1.1623^{* *}$ & 1.1656 \\
\hline & 44 & 1.2379 & 1.2166 & 1.2151 & $1.1953^{* *}$ & $1.1955^{* *}$ & 1.1976 & $1.1953^{* *}$ & 1.2025 \\
\hline & 66 & 1.2743 & 1.2488 & 1.2371 & $1.2175^{* *}$ & $1.2175^{* *}$ & 1.2205 & $1.2172^{* *}$ & 1.2283 \\
\hline \multirow{6}{*}{$\mathrm{SR}$} & 1 & $0.7881^{* * *}$ & $0.7806^{* * *}$ & $0.7872^{* * *}$ & $0.7957^{* * *}$ & $0.7947^{* * *}$ & $0.7853^{* * *}$ & $0.7947^{* * *}$ & $0.7947^{* * *}$ \\
\hline & 5 & $0.8117^{* * *}$ & $0.8070^{* * *}$ & $0.7674^{* * *}$ & $0.8145^{* * *}$ & $0.8145^{* * *}$ & $0.8079^{* * *}$ & $0.8145^{* * *}$ & $0.8070^{* * *}$ \\
\hline & 10 & $0.8183^{* * *}$ & $0.7994^{* * *}$ & $0.7429^{* * *}$ & $0.8249^{* * *}$ & $0.8202^{* * *}$ & $0.8032^{* * *}$ & $0.8173^{* * *}$ & $0.7910^{* * *}$ \\
\hline & 22 & $0.7514^{* * *}$ & $0.7137^{* * *}$ & $0.6111^{* * *}$ & $0.7533^{* * *}$ & $0.7476^{* * *}$ & $0.7109^{* * *}$ & $0.7524^{* * *}$ & $0.7128^{* * *}$ \\
\hline & 44 & $0.6177^{* * *}$ & 0.5301 & $0.5631^{* * *}$ & $0.6290^{* * *}$ & $0.6271^{* * *}$ & $0.6243^{* * *}$ & $0.6318^{* * *}$ & $0.6215^{* * *}$ \\
\hline & 66 & 0.4934 & 0.2213 & 0.4652 & 0.5122 & 0.5122 & 0.5122 & 0.5104 & 0.4802 \\
\hline
\end{tabular}

Table B.34: Forecasting Evaluation for Soy Futures with average $M e d R V_{t+h}^{(h)}$. Note that * and ** indicate the inclusion in the $\mathcal{M}_{90 \%}^{*}$ and $\mathcal{M}_{75 \%}^{*}$, respectively. For the Success Ratio (SR), the asterisk ${ }^{*},{ }^{* *}$, and *** indicate a statistical significance at $10 \%, 5 \%$, and $1 \%$, respectively. 


\begin{tabular}{|c|c|c|c|c|c|c|c|c|c|}
\hline & $h$ & RW & $\mathrm{AR}$ & ARMA & HAR & HAR-J & HAR-CJ & HAR-PS & LHAR-CJ \\
\hline \multirow{6}{*}{ MSPE } & 1 & 1.3511 & 1.2559 & 1.3330 & $1.0899^{* *}$ & $1.0989^{* *}$ & $1.0898^{* *}$ & $1.0916^{* *}$ & 1.1039 \\
\hline & 5 & 0.7882 & 0.7796 & 0.8667 & $0.7097^{* *}$ & $0.7089^{* *}$ & $0.7059^{* *}$ & $0.7089^{* *}$ & $0.7124^{* *}$ \\
\hline & 10 & $0.6749^{* *}$ & 0.6971 & 0.7766 & $0.6497^{* *}$ & $0.6496^{* *}$ & $0.6494^{* *}$ & $0.6472^{* *}$ & $0.6525^{* *}$ \\
\hline & 22 & $0.6594^{* *}$ & 0.6529 & 0.7906 & $0.6262^{* *}$ & $0.6264^{* *}$ & $0.6371^{* *}$ & $0.6254^{* *}$ & $0.6362^{* *}$ \\
\hline & 44 & $0.6311^{* *}$ & $0.6727^{*}$ & 0.7896 & $0.6395^{* *}$ & $0.6401^{* *}$ & $0.6515^{* *}$ & $0.6409^{* *}$ & $0.6446^{* *}$ \\
\hline & 66 & $0.6040^{* *}$ & 0.7083 & 0.7029 & 0.6853 & 0.6858 & 0.6865 & 0.6865 & 0.6704 \\
\hline \multirow{6}{*}{ MAPE } & 1 & 0.6343 & 0.7299 & 0.6368 & 0.6109 & $0.6001^{* *}$ & $0.5985^{* *}$ & $0.6033^{* *}$ & $0.6103^{*}$ \\
\hline & 5 & $0.5163^{* *}$ & 0.5705 & 0.5433 & 0.5154 & $0.5120^{* *}$ & $0.5084^{* *}$ & $0.5121^{* *}$ & $0.5124^{* *}$ \\
\hline & 10 & $0.4844^{* *}$ & 0.5457 & 0.5283 & $0.5034^{*}$ & $0.5014^{* *}$ & $0.4953^{* *}$ & $0.5009^{* *}$ & $0.4954^{* *}$ \\
\hline & 22 & $0.5054^{* *}$ & 0.5134 & 0.5429 & $0.4981^{* *}$ & $0.4973^{* *}$ & $0.4997^{* *}$ & $0.4963^{* *}$ & $0.5037^{* *}$ \\
\hline & 44 & $0.4912^{* *}$ & $0.4902^{* *}$ & 0.5472 & $0.5028^{* *}$ & $0.5024^{* *}$ & $0.5081^{* *}$ & $0.5014^{* *}$ & $0.5139^{* *}$ \\
\hline & 66 & $0.4736^{* *}$ & 0.4962 & $0.4878^{* *}$ & 0.5143 & 0.5145 & 0.5175 & 0.5134 & 0.5125 \\
\hline \multirow{6}{*}{ QLIKE } & 1 & 1.8012 & 1.8068 & 1.7944 & $1.7603^{* *}$ & $1.7602^{* *}$ & $1.7597^{* *}$ & $1.7608^{* *}$ & 1.9152 \\
\hline & 5 & 1.7809 & 1.7855 & 1.7894 & $1.7721^{* *}$ & $1.7717^{* *}$ & $1.7703^{* *}$ & $1.7718^{* *}$ & $1.7783^{*}$ \\
\hline & 10 & $1.7796^{* *}$ & 1.7876 & 1.7971 & $1.7799^{* *}$ & $1.7797^{* *}$ & $1.7782^{* *}$ & $1.7795^{* *}$ & $1.7848^{* *}$ \\
\hline & 22 & $1.7956^{* *}$ & 1.7952 & 1.8384 & $1.7912^{* *}$ & $1.7912^{* *}$ & $1.7917^{* *}$ & $1.7911^{* *}$ & $1.7966^{* *}$ \\
\hline & 44 & $1.8165^{* *}$ & $1.8198^{*}$ & 1.8474 & $1.8090^{* *}$ & $1.8091^{* *}$ & $1.8076^{* *}$ & $1.8091^{* *}$ & $1.8074^{* *}$ \\
\hline & 66 & $1.8145^{* *}$ & 1.8315 & $1.8276^{*}$ & $1.8246^{*}$ & $1.8245^{*}$ & $1.8192^{* *}$ & $1.8245^{*}$ & $1.8179^{* *}$ \\
\hline \multirow{6}{*}{$\mathrm{SR}$} & 1 & $0.7502^{* * *}$ & $0.7352^{* * *}$ & $0.7474^{* * *}$ & $0.7728^{* * *}$ & $0.7690^{* * *}$ & $0.7596^{* * *}$ & $0.7634^{* * *}$ & $0.7559^{* * *}$ \\
\hline & 5 & $0.7972^{* * *}$ & $0.7897^{* * *}$ & $0.8028^{* * *}$ & $0.8113^{* * *}$ & $0.8094^{* * *}$ & $0.8113^{* * *}$ & $0.8038^{* * *}$ & $0.8085^{* * *}$ \\
\hline & 10 & $0.8282^{* * *}$ & $0.8225^{* * *}$ & $0.7822^{* * *}$ & $0.8254^{* * *}$ & $0.8254^{* * *}$ & $0.8254^{* * *}$ & $0.8282^{* * *}$ & $0.8178^{* * *}$ \\
\hline & 22 & $0.8000^{* * *}$ & $0.8188^{* * *}$ & $0.8028^{* * *}$ & $0.8272^{* * *}$ & $0.8263^{* * *}$ & $0.8141^{* * *}$ & $0.8272^{* * *}$ & $0.8131^{* * *}$ \\
\hline & 44 & $0.9277^{* * *}$ & $0.8394^{* * *}$ & $0.8685^{* * *}$ & $0.8695^{* * *}$ & $0.8657^{* * *}$ & $0.8385^{* * *}$ & $0.8648^{* * *}$ & $0.8620^{* * *}$ \\
\hline & 66 & $0.9418^{* * *}$ & $0.8272^{* * *}$ & $0.8601^{* * *}$ & $0.8085^{* * *}$ & $0.8085^{* * *}$ & $0.7925^{* * *}$ & $0.8085^{* * *}$ & $0.8479^{* * *}$ \\
\hline
\end{tabular}

Table B.35: Forecasting Evaluation for Sugar Futures with average $M e d R V_{t+h}^{(h)}$. Note that * and ** indicate the inclusion in the $\mathcal{M}_{90 \%}^{*}$ and $\mathcal{M}_{75 \%}^{*}$, respectively. For the Success Ratio (SR), the asterisk *, $* *$, and $* * *$ indicate a statistical significance at $10 \%, 5 \%$, and $1 \%$, respectively.

\begin{tabular}{|c|c|c|c|c|c|c|c|c|c|}
\hline & $h$ & RW & $\mathrm{AR}$ & ARMA & HAR & HAR-J & HAR-CJ & HAR-PS & LHAR-CJ \\
\hline \multirow{6}{*}{ MSPE } & 1 & 1.2218 & 1.1210 & 1.1825 & $1.0307^{* *}$ & $1.0374^{* *}$ & $1.0375^{* *}$ & $1.0266^{* *}$ & $1.0458^{*}$ \\
\hline & 5 & 0.8464 & 0.8188 & 0.9583 & $0.7602^{*}$ & $0.7629^{*}$ & $0.7723^{*}$ & $0.7522^{* *}$ & 0.7835 \\
\hline & 10 & $0.7746^{* *}$ & $0.7530^{*}$ & $0.8184^{*}$ & $0.7146^{* *}$ & $0.7172^{* *}$ & $0.7246^{* *}$ & $0.7117^{* *}$ & $0.7305^{* *}$ \\
\hline & 22 & $0.6433^{* *}$ & $0.6860^{* *}$ & 0.7689 & $0.6682^{* *}$ & $0.6692^{* *}$ & $0.6742^{* *}$ & $0.6683^{* *}$ & $0.6810^{* *}$ \\
\hline & 44 & $0.6649^{* *}$ & $0.7111^{* *}$ & 0.9099 & $0.6943^{* *}$ & $0.6947^{* *}$ & $0.7009^{* *}$ & $0.6934^{* *}$ & $0.7125^{* *}$ \\
\hline & 66 & $0.7146^{* *}$ & 0.8147 & 1.2100 & $0.7438^{* *}$ & $0.7415^{* *}$ & $0.7333^{* *}$ & $0.7416^{* *}$ & $0.7469^{* *}$ \\
\hline \multirow{6}{*}{ MAPE } & 1 & 0.6369 & 0.6978 & 0.6358 & $0.6224^{*}$ & 0.6277 & $0.6223^{*}$ & $0.6179^{* *}$ & 0.6402 \\
\hline & 5 & $0.5194^{* *}$ & 0.5659 & 0.5526 & $0.5284^{*}$ & 0.5312 & $0.5302 *$ & $0.5253^{* *}$ & 0.5473 \\
\hline & 10 & $0.4911^{* *}$ & 0.5327 & $0.5246^{*}$ & $0.5173^{*}$ & 0.5189 & $0.5157^{*}$ & $0.5160^{*}$ & 0.5332 \\
\hline & 22 & $0.4704^{* *}$ & 0.5298 & 0.5557 & 0.5180 & 0.5189 & 0.5155 & 0.5177 & 0.5384 \\
\hline & 44 & $0.4968^{* *}$ & 0.5586 & 0.6352 & 0.5510 & 0.5509 & 0.5489 & 0.5502 & 0.5665 \\
\hline & 66 & $0.5213^{* *}$ & 0.6044 & 0.7160 & 0.5800 & 0.5788 & 0.5740 & 0.5788 & 0.5916 \\
\hline \multirow{6}{*}{ QLIKE } & 1 & 1.7837 & 1.7570 & 1.7640 & $1.7348^{* *}$ & $1.7354^{* *}$ & $1.7351^{* *}$ & $1.7342^{* *}$ & 1.7384 \\
\hline & 5 & 1.7570 & 1.7588 & 1.7702 & $1.7487^{*}$ & $1.7487^{*}$ & $1.7491^{*}$ & $1.7478^{* *}$ & 1.7522 \\
\hline & 10 & $1.7606^{* *}$ & 1.7631 & 1.7676 & $1.7585^{* *}$ & $1.7587^{* *}$ & $1.7587^{* *}$ & $1.7581^{* *}$ & $1.7606^{* *}$ \\
\hline & 22 & $1.7643^{* *}$ & 1.7714 & 1.7917 & $1.7688^{* *}$ & $1.7688^{* *}$ & $1.7689^{* *}$ & $1.7687^{* *}$ & $1.7701^{* *}$ \\
\hline & 44 & $1.7898^{* *}$ & 1.7926 & 1.8220 & $1.7855^{* *}$ & $1.7853^{* *}$ & $1.7876^{* *}$ & $1.7853^{* *}$ & $1.7867^{* *}$ \\
\hline & 66 & $1.8105^{* *}$ & 1.8116 & 1.8903 & $1.7997^{* *}$ & $1.7994^{* *}$ & $1.8002^{* *}$ & $1.7995^{* *}$ & $1.7988^{* *}$ \\
\hline \multirow{6}{*}{ SR } & 1 & $0.7030^{* * *}$ & $0.6635^{* * *}$ & $0.7002^{* * *}$ & $0.7049^{* * *}$ & $0.7039^{* * *}$ & $0.7068^{* * *}$ & $0.7115^{* * *}$ & $0.7105^{* * *}$ \\
\hline & 5 & $0.7491^{* * *}$ & $0.6729^{* * *}$ & $0.7115^{* * *}$ & $0.7434^{* * *}$ & $0.7265^{* * *}$ & $0.7246^{* * *}$ & $0.7378^{* * *}$ & $0.7397^{* * *}$ \\
\hline & 10 & $0.7716^{* * *}$ & $0.6880^{* * *}$ & $0.7237^{* * *}$ & $0.7415^{* * *}$ & $0.7453^{* * *}$ & $0.7293^{* * *}$ & $0.7434^{* * *}$ & $0.7509^{* * *}$ \\
\hline & 22 & $0.8205^{* * *}$ & $0.6945^{* * *}$ & $0.6353^{* * *}$ & $0.7171^{* * *}$ & $0.7180^{* * *}$ & $0.7115^{* * *}$ & $0.7143^{* * *}$ & $0.7472^{* * *}$ \\
\hline & 44 & $0.7491^{* * *}$ & $0.5836^{* * *}$ & 0.3703 & $0.6297^{* * *}$ & $0.6344^{* * *}$ & $0.6335^{* * *}$ & $0.6297^{* * *}$ & $0.6720^{* * *}$ \\
\hline & 66 & $0.6551^{* * *}$ & 0.3853 & 0.3449 & 0.5122 & 0.5179 & $0.5254^{* *}$ & 0.5122 & $0.5677^{* * *}$ \\
\hline
\end{tabular}

Table B.36: Forecasting Evaluation for Wheat Futures with average $M e d R V_{t+h}^{(h)}$. Note that * and ** indicate the inclusion in the $\mathcal{M}_{90 \%}^{*}$ and $\mathcal{M}_{75 \%}^{*}$, respectively. For the Success Ratio (SR), the asterisk *, $* *$, and $* * *$ indicate a statistical significance at $10 \%, 5 \%$, and $1 \%$, respectively. 


\section{Appendix C. Value-at-Risk Backtesting Results}

\begin{tabular}{|c|c|c|c|c|c|c|c|c|c|c|c|c|c|}
\hline \multirow{3}{*}{$\begin{array}{l}\alpha \\
\text { Position } \\
\text { Test }\end{array}$} & & \multicolumn{4}{|c|}{0.01} & \multicolumn{4}{|c|}{0.025} & \multicolumn{4}{|c|}{0.05} \\
\hline & & \multicolumn{2}{|c|}{ long } & \multicolumn{2}{|c|}{ short } & \multicolumn{2}{|c|}{ long } & \multicolumn{2}{|c|}{ short } & \multicolumn{2}{|c|}{ long } & \multicolumn{2}{|c|}{ short } \\
\hline & & $\mathrm{UC}$ & $\mathrm{CC}$ & $\mathrm{UC}$ & $\mathrm{CC}$ & $\mathrm{UC}$ & $\mathrm{CC}$ & UC & $\mathrm{CC}$ & $\mathrm{UC}$ & $\mathrm{CC}$ & UC & $\mathrm{CC}$ \\
\hline \multirow{6}{*}{ RW } & 1 & 0.0004 & 0.0010 & 0.0020 & 0.0054 & 0.0205 & 0.0593 & 0.0735 & 0.0567 & 0.2083 & 0.3288 & 0.6596 & 0.7190 \\
\hline & 5 & 0.0095 & 0.0234 & 0.0009 & 0.0023 & 0.3801 & 0.3905 & 0.0205 & 0.0310 & 0.6596 & 0.2182 & 0.5628 & 0.4325 \\
\hline & 10 & 0.0045 & 0.0032 & 0.0004 & 0.0015 & 0.1073 & 0.0867 & 0.0205 & 0.0310 & 0.0973 & 0.0813 & 0.3938 & 0.4113 \\
\hline & 22 & 0.0020 & 0.0018 & 0.0000 & 0.0000 & 0.1073 & 0.0249 & 0.0003 & 0.0005 & 0.2083 & 0.0048 & 0.1272 & 0.0850 \\
\hline & 44 & 0.0001 & 0.0000 & 0.0000 & 0.0001 & 0.0128 & 0.0001 & 0.0003 & 0.0005 & 0.1639 & 0.0051 & 0.2083 & 0.1949 \\
\hline & 66 & 0.0001 & 0.0000 & 0.0001 & 0.0002 & 0.0015 & 0.0000 & 0.0005 & 0.0016 & 0.0048 & 0.0021 & 0.3228 & 0.5413 \\
\hline \multirow{6}{*}{ AR } & 1 & 0.0678 & 0.1017 & 0.0368 & 0.0814 & 0.1527 & 0.2514 & 0.1527 & 0.1162 & 0.4739 & 0.1014 & 0.9043 & 0.8877 \\
\hline & 5 & 0.0368 & 0.0664 & 0.0020 & 0.0053 & 0.3801 & 0.3905 & 0.1527 & 0.2440 & 0.8715 & 0.1831 & 0.8715 & 0.1707 \\
\hline & 10 & 0.0045 & 0.0032 & 0.0001 & 0.0006 & 0.1527 & 0.1014 & 0.0322 & 0.0830 & 0.4739 & 0.0111 & 0.6596 & 0.7014 \\
\hline & 22 & 0.0045 & 0.0032 & 0.0001 & 0.0004 & 0.1527 & 0.0008 & 0.0027 & 0.0104 & 0.5628 & 0.0327 & 0.3938 & 0.2227 \\
\hline & 44 & 0.0020 & 0.0018 & 0.0045 & 0.0113 & 0.1527 & 0.0008 & 0.0205 & 0.0583 & 0.3938 & 0.0125 & 0.6596 & 0.4278 \\
\hline & 66 & 0.0191 & 0.0406 & 0.0678 & 0.1405 & 0.3801 & 0.0044 & 0.1527 & 0.3474 & 0.9043 & 0.0460 & 0.3233 & 0.4517 \\
\hline \multirow{6}{*}{ ARMA } & 1 & 0.0020 & 0.0053 & 0.0095 & 0.0235 & 0.0492 & 0.1168 & 0.0735 & 0.0567 & 0.3938 & 0.4254 & 0.8715 & 0.8368 \\
\hline & 5 & 0.0095 & 0.0053 & 0.0045 & 0.0113 & 0.1073 & 0.2016 & 0.1073 & 0.1962 & 0.5628 & 0.2293 & 0.6596 & 0.2048 \\
\hline & 10 & 0.0020 & 0.0018 & 0.0001 & 0.0002 & 0.2121 & 0.0286 & 0.0005 & 0.0003 & 0.3228 & 0.0138 & 0.4739 & 0.2220 \\
\hline & 22 & 0.0004 & 0.0005 & 0.0020 & 0.0054 & 0.0735 & 0.0008 & 0.0078 & 0.0262 & 0.3938 & 0.0395 & 0.2611 & 0.2086 \\
\hline & 44 & 0.0191 & 0.0406 & 0.0678 & 0.1405 & 0.2121 & 0.0007 & 0.6174 & 0.8301 & 0.7929 & 0.0096 & 0.4005 & 0.5414 \\
\hline & 66 & 0.0678 & 0.1017 & 0.0368 & 0.0814 & 0.6174 & 0.0030 & 0.2875 & 0.5496 & 0.2561 & 0.0050 & 0.7929 & 0.2772 \\
\hline \multirow{6}{*}{ HAR } & 1 & 0.0678 & 0.1405 & 0.0095 & 0.0235 & 0.1527 & 0.1014 & 0.1527 & 0.1162 & 0.3938 & 0.1056 & 0.8715 & 0.9211 \\
\hline & 5 & 0.0191 & 0.0083 & 0.0020 & 0.0053 & 0.3801 & 0.1282 & 0.1073 & 0.2622 & 0.8715 & 0.1831 & 0.9831 & 0.3564 \\
\hline & 10 & 0.0020 & 0.0018 & 0.0009 & 0.0023 & 0.0492 & 0.0569 & 0.1527 & 0.2440 & 0.5628 & 0.0095 & 0.3228 & 0.2181 \\
\hline & 22 & 0.0095 & 0.0053 & 0.0001 & 0.0002 & 0.0735 & 0.0008 & 0.0047 & 0.0168 & 0.4739 & 0.0364 & 0.3938 & 0.2227 \\
\hline & 44 & 0.0009 & 0.0010 & 0.0009 & 0.0023 & 0.1073 & 0.0052 & 0.0205 & 0.0583 & 0.6596 & 0.0286 & 0.3938 & 0.2227 \\
\hline & 66 & 0.0004 & 0.0005 & 0.0045 & 0.0115 & 0.2121 & 0.0007 & 0.0322 & 0.0416 & 0.4739 & 0.0111 & 0.7929 & 0.8546 \\
\hline \multirow{6}{*}{ HAR-J } & 1 & 0.1187 & 0.2279 & 0.0191 & 0.0445 & 0.1527 & 0.1014 & 0.1073 & 0.0824 & 0.3228 & 0.1073 & 0.8715 & 0.9211 \\
\hline & 5 & 0.0191 & 0.0083 & 0.0020 & 0.0053 & 0.3801 & 0.1282 & 0.1073 & 0.2622 & 0.7630 & 0.2025 & 0.9043 & 0.3185 \\
\hline & 10 & 0.0045 & 0.0032 & 0.0009 & 0.0023 & 0.0492 & 0.0569 & 0.1527 & 0.2440 & 0.5628 & 0.0095 & 0.3228 & 0.2181 \\
\hline & 22 & 0.0095 & 0.0053 & 0.0001 & 0.0002 & 0.0735 & 0.0008 & 0.0047 & 0.0168 & 0.4739 & 0.0364 & 0.4739 & 0.2220 \\
\hline & 44 & 0.0009 & 0.0010 & 0.0009 & 0.0023 & 0.1073 & 0.0052 & 0.0205 & 0.0583 & 0.6596 & 0.0286 & 0.4739 & 0.4268 \\
\hline & 66 & 0.0009 & 0.0010 & 0.0045 & 0.0115 & 0.2121 & 0.0007 & 0.0322 & 0.0416 & 0.5628 & 0.0095 & 0.9043 & 0.8981 \\
\hline \multirow{6}{*}{ HAR-CJ } & 1 & 0.0678 & 0.1405 & 0.0095 & 0.0230 & 0.1527 & 0.2514 & 0.1527 & 0.1162 & 0.2611 & 0.1064 & 0.9831 & 0.9207 \\
\hline & 5 & 0.0191 & 0.0083 & 0.0020 & 0.0053 & 0.4904 & 0.4184 & 0.1073 & 0.2622 & 0.6596 & 0.2182 & 0.5628 & 0.2159 \\
\hline & 10 & 0.0020 & 0.0018 & 0.0020 & 0.0053 & 0.0492 & 0.0569 & 0.1527 & 0.2440 & 0.5628 & 0.0095 & 0.4739 & 0.2220 \\
\hline & 22 & 0.0095 & 0.0053 & 0.0000 & 0.0001 & 0.1073 & 0.0249 & 0.0078 & 0.0262 & 0.3228 & 0.0418 & 0.3938 & 0.2227 \\
\hline & 44 & 0.0009 & 0.0010 & 0.0045 & 0.0115 & 0.2121 & 0.0286 & 0.0128 & 0.0397 & 0.8715 & 0.0201 & 0.4739 & 0.4268 \\
\hline & 66 & 0.0009 & 0.0010 & 0.0095 & 0.0235 & 0.2121 & 0.0007 & 0.1073 & 0.1962 & 0.6596 & 0.0080 & 0.6849 & 0.7930 \\
\hline \multirow{6}{*}{ HAR-PS } & 1 & 0.1187 & 0.2279 & 0.0191 & 0.0445 & 0.2121 & 0.1141 & 0.1527 & 0.1162 & 0.4739 & 0.1014 & 0.7630 & 0.7057 \\
\hline & 5 & 0.0191 & 0.0083 & 0.0020 & 0.0053 & 0.4904 & 0.4184 & 0.1073 & 0.2622 & 0.7630 & 0.0770 & 0.8715 & 0.1707 \\
\hline & 10 & 0.0045 & 0.0032 & 0.0009 & 0.0023 & 0.0735 & 0.0715 & 0.1073 & 0.1962 & 0.5628 & 0.0095 & 0.3228 & 0.2181 \\
\hline & 22 & 0.0095 & 0.0053 & 0.0001 & 0.0002 & 0.1073 & 0.0052 & 0.0047 & 0.0168 & 0.5628 & 0.0327 & 0.3228 & 0.2181 \\
\hline & 44 & 0.0009 & 0.0010 & 0.0020 & 0.0053 & 0.1073 & 0.0052 & 0.0205 & 0.0583 & 0.5628 & 0.0327 & 0.3228 & 0.2181 \\
\hline & 66 & 0.0009 & 0.0010 & 0.0045 & 0.0115 & 0.2121 & 0.0007 & 0.0322 & 0.0416 & 0.3938 & 0.0125 & 0.7929 & 0.8546 \\
\hline & 1 & 0.0368 & 0.0814 & 0.0095 & 0.0230 & 0.4904 & 0.7562 & 0.1527 & 0.1162 & 0.2611 & 0.2188 & 0.7630 & 0.7057 \\
\hline & 5 & 0.0191 & 0.0083 & 0.0045 & 0.0113 & 0.4904 & 0.4184 & 0.1073 & 0.2622 & 0.4739 & 0.4427 & 0.8715 & 0.1707 \\
\hline & 10 & 0.0045 & 0.0032 & 0.0045 & 0.0113 & 0.1073 & 0.0867 & 0.2121 & 0.2925 & 0.5628 & 0.0095 & 0.3228 & 0.1000 \\
\hline LHAR-CJ & 22 & 0.0095 & 0.0053 & 0.0001 & 0.0004 & 0.0735 & 0.0049 & 0.0078 & 0.0262 & 0.4739 & 0.0364 & 0.3938 & 0.2227 \\
\hline & 44 & 0.0009 & 0.0010 & 0.0045 & 0.0115 & 0.3801 & 0.0044 & 0.0078 & 0.0262 & 0.6596 & 0.0286 & 0.4739 & 0.4268 \\
\hline & 66 & 0.0001 & 0.0000 & 0.0191 & 0.0453 & 0.2121 & 0.0001 & 0.0492 & 0.1143 & 0.7630 & 0.0065 & 0.5825 & 0.7170 \\
\hline
\end{tabular}

Table C.37: Value-at-Risk backtesting results for $R V$ for Corn Futures. Note that UC is the unconditional coverage test by Kupied ([995), $\mathrm{CC}$ is the conditional coverage test by Christoffersen (ए998), $\alpha$ is the Value-at-Risk level, Position corresponds with the trading position, which is either long (risk of rising prices) or short (risk of falling prices). The test results are presented as $p$-values from the UC and CC test for the forecast horizons 1-, 5-, 10-, 22-, 44-, and 66-days ahead. 


\begin{tabular}{|c|c|c|c|c|c|c|c|c|c|c|c|c|c|}
\hline \multirow{3}{*}{$\begin{array}{l}\alpha \\
\text { Position } \\
\text { Test }\end{array}$} & & \multicolumn{4}{|c|}{0.01} & \multicolumn{4}{|c|}{0.025} & \multicolumn{4}{|c|}{0.05} \\
\hline & & \multicolumn{2}{|c|}{ long } & \multicolumn{2}{|c|}{ short } & \multicolumn{2}{|c|}{ long } & \multicolumn{2}{|c|}{ short } & \multicolumn{2}{|c|}{ long } & \multicolumn{2}{|c|}{ short } \\
\hline & & $\mathrm{UC}$ & $\mathrm{CC}$ & $\mathrm{UC}$ & $\mathrm{CC}$ & $\mathrm{UC}$ & $\mathrm{CC}$ & $\mathrm{UC}$ & $\mathrm{CC}$ & $\mathrm{UC}$ & $\mathrm{CC}$ & $\mathrm{UC}$ & $\mathrm{CC}$ \\
\hline \multirow{6}{*}{ RW } & 1 & 0.3078 & 0.4857 & 0.1942 & 0.3417 & 0.7472 & 0.8862 & 0.4809 & 0.3144 & 0.2648 & 0.5133 & 0.1175 & 0.2377 \\
\hline & 5 & 0.4115 & 0.0980 & 0.8876 & 0.2532 & 0.0487 & 0.0124 & 0.0487 & 0.0775 & 0.0017 & 0.0004 & 0.0291 & 0.0778 \\
\hline & 10 & 0.4115 & 0.0980 & 0.3078 & 0.2345 & 0.0149 & 0.0028 & 0.6379 & 0.2550 & 0.0048 & 0.0003 & 0.0613 & 0.0899 \\
\hline & 22 & 0.6248 & 0.0057 & 0.3078 & 0.2345 & 0.0487 & 0.0124 & 0.6379 & 0.2550 & 0.0017 & 0.0016 & 0.1175 & 0.2730 \\
\hline & 44 & 0.6583 & 0.2724 & 0.8661 & 0.8872 & 0.2741 & 0.0138 & 0.2741 & 0.1013 & 0.0009 & 0.0008 & 0.1573 & 0.0370 \\
\hline & 66 & 0.6583 & 0.2724 & 0.4625 & 0.6409 & 0.2741 & 0.0138 & 0.6379 & 0.2550 & 0.0009 & 0.0002 & 0.5003 & 0.3870 \\
\hline \multirow{6}{*}{$\mathrm{AR}$} & 1 & 0.8661 & 0.8872 & 0.0663 & 0.0160 & 0.1273 & 0.2165 & 0.8984 & 0.4005 & 0.0125 & 0.0343 & 0.2062 & 0.1489 \\
\hline & 5 & 0.6248 & 0.1542 & 0.8661 & 0.2104 & 0.0075 & 0.0011 & 0.1910 & 0.2889 & 0.0029 & 0.0029 & 0.0613 & 0.1549 \\
\hline & 10 & 0.4115 & 0.0980 & 0.1942 & 0.1907 & 0.0149 & 0.0028 & 0.4995 & 0.1982 & 0.0009 & 0.0002 & 0.0291 & 0.0403 \\
\hline & 22 & 0.8661 & 0.0100 & 0.4625 & 0.6409 & 0.0808 & 0.0233 & 0.4995 & 0.1982 & 0.0009 & 0.0008 & 0.3335 & 0.5992 \\
\hline & 44 & 0.4625 & 0.2648 & 0.6583 & 0.7807 & 0.2741 & 0.0138 & 0.3772 & 0.1458 & 0.0009 & 0.0008 & 0.1573 & 0.0370 \\
\hline & 66 & 0.3078 & 0.2345 & 0.3078 & 0.4857 & 0.7882 & 0.0631 & 0.7882 & 0.3113 & 0.0125 & 0.0048 & 0.7004 & 0.5268 \\
\hline \multirow{6}{*}{ ARMA } & 1 & 0.3078 & 0.4857 & 0.1942 & 0.3417 & 0.9448 & 0.8890 & 0.4809 & 0.3144 & 0.2062 & 0.4278 & 0.0613 & 0.1499 \\
\hline & 5 & 0.6248 & 0.1542 & 0.6583 & 0.7807 & 0.0487 & 0.0124 & 0.6379 & 0.2550 & 0.0079 & 0.0006 & 0.0193 & 0.0525 \\
\hline & 10 & 0.8661 & 0.2104 & 0.8876 & 0.2532 & 0.0808 & 0.0233 & 0.7882 & 0.3113 & 0.0029 & 0.0029 & 0.0613 & 0.0899 \\
\hline & 22 & 0.6248 & 0.8141 & 0.6583 & 0.7807 & 0.6379 & 0.2550 & 0.9448 & 0.3617 & 0.0009 & 0.0008 & 0.0858 & 0.0530 \\
\hline & 44 & 0.3078 & 0.2345 & 0.4625 & 0.6409 & 0.4995 & 0.0332 & 0.9448 & 0.3617 & 0.0291 & 0.0140 & 0.7004 & 0.5268 \\
\hline & 66 & 0.6583 & 0.7807 & 0.0359 & 0.0651 & 0.6379 & 0.0471 & 0.0474 & 0.1135 & 0.1573 & 0.0004 & 0.3819 & 0.4198 \\
\hline \multirow{6}{*}{ HAR } & 1 & 0.0560 & 0.0082 & 0.8876 & 0.8723 & 0.0035 & 0.0049 & 0.2741 & 0.1013 & 0.0125 & 0.0158 & 0.1175 & 0.1766 \\
\hline & 5 & 0.4115 & 0.0980 & 0.8876 & 0.2532 & 0.0035 & 0.0049 & 0.1273 & 0.1983 & 0.0029 & 0.0007 & 0.0613 & 0.1549 \\
\hline & 10 & 0.4115 & 0.0980 & 0.8876 & 0.2532 & 0.0149 & 0.0028 & 0.4995 & 0.1982 & 0.0009 & 0.0002 & 0.0428 & 0.0612 \\
\hline & 22 & 0.4115 & 0.0980 & 0.8876 & 0.8723 & 0.1910 & 0.0662 & 0.2741 & 0.1013 & 0.0009 & 0.0008 & 0.5003 & 0.6548 \\
\hline & 44 & 0.6248 & 0.8141 & 0.8876 & 0.8723 & 0.1273 & 0.0406 & 0.7882 & 0.3113 & 0.0001 & 0.0001 & 0.2648 & 0.1975 \\
\hline & 66 & 0.6583 & 0.2724 & 0.6583 & 0.7807 & 0.2741 & 0.0138 & 0.7882 & 0.0631 & 0.0029 & 0.0001 & 0.5003 & 0.1696 \\
\hline \multirow{6}{*}{ HAR-J } & 1 & 0.1262 & 0.0235 & 0.8876 & 0.8723 & 0.0015 & 0.0020 & 0.2741 & 0.1013 & 0.0125 & 0.0158 & 0.0613 & 0.0899 \\
\hline & 5 & 0.4115 & 0.0980 & 0.8876 & 0.2532 & 0.0035 & 0.0049 & 0.1910 & 0.2889 & 0.0017 & 0.0004 & 0.0613 & 0.1549 \\
\hline & 10 & 0.4115 & 0.0980 & 0.6583 & 0.2724 & 0.0149 & 0.0028 & 0.4995 & 0.1982 & 0.0005 & 0.0001 & 0.0428 & 0.0612 \\
\hline & 22 & 0.2431 & 0.0529 & 0.8876 & 0.8723 & 0.1910 & 0.0662 & 0.2741 & 0.1013 & 0.0005 & 0.0004 & 0.5969 & 0.7392 \\
\hline & 44 & 0.6248 & 0.8141 & 0.8876 & 0.8723 & 0.1273 & 0.0406 & 0.7882 & 0.3113 & 0.0003 & 0.0002 & 0.2648 & 0.1975 \\
\hline & 66 & 0.6583 & 0.2724 & 0.6583 & 0.7807 & 0.2741 & 0.0138 & 0.7882 & 0.0631 & 0.0029 & 0.0001 & 0.4122 & 0.1329 \\
\hline \multirow{6}{*}{ HAR-CJ } & 1 & 0.1262 & 0.0235 & 0.8876 & 0.8723 & 0.0035 & 0.0049 & 0.3772 & 0.1458 & 0.0029 & 0.0078 & 0.0613 & 0.0899 \\
\hline & 5 & 0.4115 & 0.0980 & 0.8661 & 0.2104 & 0.0075 & 0.0111 & 0.0487 & 0.0775 & 0.0009 & 0.0002 & 0.0428 & 0.1115 \\
\hline & 10 & 0.4115 & 0.0980 & 0.6583 & 0.2724 & 0.0278 & 0.0061 & 0.2741 & 0.1013 & 0.0017 & 0.0004 & 0.0291 & 0.0403 \\
\hline & 22 & 0.4115 & 0.0980 & 0.4625 & 0.2648 & 0.2741 & 0.1013 & 0.2741 & 0.1013 & 0.0029 & 0.0029 & 0.3335 & 0.5992 \\
\hline & 44 & 0.8876 & 0.8723 & 0.8876 & 0.8723 & 0.1910 & 0.0080 & 0.8984 & 0.0966 & 0.0005 & 0.0004 & 0.5003 & 0.3870 \\
\hline & 66 & 0.8876 & 0.2532 & 0.6583 & 0.7807 & 0.1910 & 0.0662 & 0.9448 & 0.0801 & 0.0017 & 0.0004 & 0.2062 & 0.0534 \\
\hline \multirow{6}{*}{ HAR-PS } & 1 & 0.0560 & 0.0082 & 0.6583 & 0 & 35 & 049 & 772 & 221 & 0.0079 & 093 & 0.0428 & 0.0612 \\
\hline & 5 & 0.4115 & 0.0980 & 0.8876 & 0.2532 & 0.0035 & 0.0049 & 0.1910 & 0.0662 & 0.0029 & 0.0007 & 0.0858 & 0.2088 \\
\hline & 10 & 0.4115 & 0.0980 & 0.8876 & 0.2532 & 0.0149 & 0.0028 & 0.4995 & 0.1982 & 0.0009 & 0.0002 & 0.0193 & 0.0256 \\
\hline & 22 & 0.4115 & 0.0980 & 0.8876 & 0.8723 & 0.1910 & 0.0662 & 0.2741 & 0.1013 & 0.0005 & 0.0004 & 0.5003 & 0.6548 \\
\hline & 44 & 0.4115 & 0.6663 & 0.8876 & 0.8723 & 0.1273 & 0.0406 & 0.9448 & 0.3617 & 0.0003 & 0.0002 & 0.2062 & 0.1489 \\
\hline & 66 & 0.6583 & 0.2724 & 0.4625 & 0.6409 & 0.3772 & 0.0221 & 0.7882 & 0.0631 & 0.0017 & 0.0004 & 0.4122 & 0.1329 \\
\hline & 1 & 0.1262 & 0.0235 & 0.4625 & 0.6409 & 0.0035 & 0.0049 & 0.2741 & 0.3969 & 0.0029 & 0.0078 & 0.0613 & 0.0899 \\
\hline & 5 & 0.2431 & 0.0529 & 0.6248 & 0.8141 & 0.0075 & 0.0111 & 0.0278 & 0.0437 & 0.0005 & 0.0001 & 0.0193 & 0.0525 \\
\hline & 10 & 0.2431 & 0.0529 & 0.8876 & 0.8723 & 0.0075 & 0.0011 & 0.0808 & 0.0233 & 0.0017 & 0.0004 & 0.0428 & 0.1115 \\
\hline$L$ & 22 & 0.0560 & 0.0082 & 0.4625 & 0.2648 & 0.0808 & 0.1280 & 0.1273 & 0.0406 & 0.0009 & 0.0008 & 0.1175 & 0.2730 \\
\hline & 44 & 0.6248 & 0.8141 & 0.6583 & 0.2724 & 0.1910 & 0.0662 & 0.4995 & 0.1982 & 0.0003 & 0.0002 & 0.0613 & 0.0899 \\
\hline & 66 & 0.2431 & 0.0529 & 0.6583 & 0.7807 & 0.1273 & 0.0406 & 0.2741 & 0.1013 & 0.0017 & 0.0001 & 0.0291 & 0.0140 \\
\hline
\end{tabular}

Table C.38: Value-at-Risk backtesting results for $R V$ for Rice Futures. Note that UC is the unconditional coverage test by Kupied ([1995), CC is the conditional coverage test by Christoffersen (I.998), $\alpha$ is the Value-at-Risk level, Position corresponds with the trading position, which is either long (risk of rising prices) or short (risk of falling prices). The test results are presented as $p$-values from the UC and CC test for the forecast horizons 1-, 5-, 10-, 22-, 44-, and 66-days ahead. 


\begin{tabular}{|c|c|c|c|c|c|c|c|c|c|c|c|c|c|}
\hline \multirow{3}{*}{$\begin{array}{l}\alpha \\
\text { Position } \\
\text { Test }\end{array}$} & & \multirow{2}{*}{\multicolumn{4}{|c|}{0.01}} & \multicolumn{4}{|c|}{0.025} & \multicolumn{4}{|c|}{0.05} \\
\hline & & & & & & \multicolumn{2}{|c|}{ long } & \multicolumn{2}{|c|}{ short } & \multicolumn{2}{|c|}{ long } & \multicolumn{2}{|c|}{ short } \\
\hline & & UC & $\mathrm{CC}$ & $\mathrm{UC}$ & $\mathrm{CC}$ & UC & $\mathrm{CC}$ & $\mathrm{UC}$ & $\mathrm{CC}$ & $\mathrm{UC}$ & $\mathrm{CC}$ & $\mathrm{UC}$ & $\mathrm{CC}$ \\
\hline \multirow{6}{*}{ RW } & 1 & 0.0000 & 0.0000 & 0.0009 & 0.0025 & 0.0051 & 0.0182 & 0.0343 & 0.0981 & 0.1050 & 0.2198 & 0.3408 & 0.5877 \\
\hline & 5 & 0.0000 & 0.0001 & 0.0200 & 0.0406 & 0.0523 & 0.0193 & 0.0523 & 0.1199 & 0.1752 & 0.3715 & 0.2767 & 0.3679 \\
\hline & 10 & 0.0002 & 0.0003 & 0.0000 & 0.0000 & 0.0778 & 0.0742 & 0.0523 & 0.0563 & 0.3408 & 0.4103 & 0.2217 & 0.3327 \\
\hline & 22 & 0.0001 & 0.0001 & 0.0009 & 0.0010 & 0.0220 & 0.0342 & 0.0001 & 0.0003 & 0.0230 & 0.0213 & 0.0796 & 0.0717 \\
\hline & 44 & 0.0000 & 0.0000 & 0.0000 & 0.0000 & 0.0005 & 0.0004 & 0.0030 & 0.0033 & 0.0163 & 0.0087 & 0.0230 & 0.0364 \\
\hline & 66 & 0.0000 & 0.0000 & 0.0000 & 0.0000 & 0.0001 & 0.0001 & 0.0005 & 0.0001 & 0.0036 & 0.0038 & 0.1365 & 0.0883 \\
\hline \multirow{6}{*}{$\mathrm{AR}$} & 1 & 0.0001 & 0.0001 & 0.0047 & 0.0121 & 0.0138 & 0.0429 & 0.2216 & 0.4591 & 0.1752 & 0.3013 & 0.4141 & 0.6705 \\
\hline & 5 & 0.0004 & 0.0001 & 0.0384 & 0.0657 & 0.0343 & 0.0458 & 0.0343 & 0.0875 & 0.2767 & 0.5025 & 0.4964 & 0.4335 \\
\hline & 10 & 0.0009 & 0.0010 & 0.0009 & 0.0010 & 0.0523 & 0.0593 & 0.0220 & 0.0327 & 0.4141 & 0.4346 & 0.4964 & 0.4335 \\
\hline & 22 & 0.0000 & 0.0001 & 0.0022 & 0.0018 & 0.0343 & 0.0458 & 0.0523 & 0.1199 & 0.0796 & 0.0368 & 0.2767 & 0.2143 \\
\hline & 44 & 0.0000 & 0.0000 & 0.0001 & 0.0001 & 0.0017 & 0.0024 & 0.0220 & 0.0116 & 0.2767 & 0.2248 & 0.2767 & 0.2143 \\
\hline & 66 & 0.0000 & 0.0000 & 0.0004 & 0.0011 & 0.0017 & 0.0024 & 0.0778 & 0.1590 & 0.3408 & 0.2344 & 0.4141 & 0.2262 \\
\hline \multirow{6}{*}{ ARMA } & 1 & 0.0000 & 0.0000 & 0.0009 & 0.0025 & 0.0085 & 0.0284 & 0.0220 & 0.0655 & 0.1050 & 0.2198 & 0.3408 & 0.5877 \\
\hline & 5 & 0.0009 & 0.0010 & 0.0100 & 0.0236 & 0.0343 & 0.0458 & 0.0343 & 0.0875 & 0.2217 & 0.4367 & 0.2767 & 0.3679 \\
\hline & 10 & 0.0000 & 0.0001 & 0.0004 & 0.0015 & 0.0343 & 0.0458 & 0.0343 & 0.0875 & 0.1365 & 0.0936 & 0.3408 & 0.5588 \\
\hline & 22 & 0.0000 & 0.0000 & 0.0001 & 0.0001 & 0.0010 & 0.0006 & 0.0523 & 0.0177 & 0.1050 & 0.0850 & 0.2767 & 0.3679 \\
\hline & 44 & 0.0001 & 0.0001 & 0.0100 & 0.0241 & 0.0220 & 0.0125 & 0.0778 & 0.1590 & 0.4141 & 0.2388 & 0.4964 & 0.2243 \\
\hline & 66 & 0.0000 & 0.0001 & 0.0009 & 0.0034 & 0.0051 & 0.0016 & 0.0778 & 0.1590 & 0.3408 & 0.1090 & 0.4964 & 0.4335 \\
\hline \multirow{6}{*}{ HAR } & 1 & 0.0009 & 0.0034 & 0.0100 & 0.0246 & 0.0138 & 0.0247 & 0.1130 & 0.2036 & 0.1050 & 0.1513 & 0.4141 & 0.6705 \\
\hline & 5 & 0.0002 & 0.0003 & 0.0384 & 0.0657 & 0.0343 & 0.0458 & 0.0523 & 0.1199 & 0.4141 & 0.6225 & 0.4964 & 0.6588 \\
\hline & 10 & 0.0009 & 0.0010 & 0.0047 & 0.0031 & 0.0523 & 0.0593 & 0.0343 & 0.0875 & 0.3408 & 0.5656 & 0.5872 & 0.4368 \\
\hline & 22 & 0.0000 & 0.0000 & 0.0047 & 0.0129 & 0.0138 & 0.0247 & 0.0523 & 0.1199 & 0.0440 & 0.0292 & 0.2767 & 0.4976 \\
\hline & 44 & 0.0000 & 0.0000 & 0.0001 & 0.0001 & 0.0051 & 0.0051 & 0.0523 & 0.0563 & 0.1050 & 0.0850 & 0.2767 & 0.2143 \\
\hline & 66 & 0.0000 & 0.0000 & 0.0002 & 0.0002 & 0.0010 & 0.0006 & 0.1602 & 0.2519 & 0.2767 & 0.2248 & 0.3408 & 0.2228 \\
\hline \multirow{6}{*}{ HAR-J } & 1 & 0.0009 & 0.0034 & 0.0100 & 0.0246 & 0.0138 & 0.0247 & 0.1130 & 0.2036 & 0.2217 & 0.2106 & 0.3408 & 0.5877 \\
\hline & 5 & 0.0002 & 0.0003 & 0.0384 & 0.0657 & 0.0220 & 0.0342 & 0.0343 & 0.0875 & 0.3408 & 0.5656 & 0.4964 & 0.6588 \\
\hline & 10 & 0.0009 & 0.0010 & 0.0047 & 0.0031 & 0.0343 & 0.0458 & 0.0343 & 0.0875 & 0.2767 & 0.5025 & 0.5872 & 0.4368 \\
\hline & 22 & 0.0000 & 0.0000 & 0.0022 & 0.0018 & 0.0085 & 0.0173 & 0.0778 & 0.1590 & 0.0440 & 0.0292 & 0.2767 & 0.4976 \\
\hline & 44 & 0.0000 & 0.0000 & 0.0001 & 0.0001 & 0.0051 & 0.0051 & 0.0523 & 0.0563 & 0.1050 & 0.0850 & 0.2767 & 0.2143 \\
\hline & 66 & 0.0000 & 0.0000 & 0.0002 & 0.0002 & 0.0010 & 0.0006 & 0.1130 & 0.2036 & 0.2767 & 0.2248 & 0.3408 & 0.2228 \\
\hline \multirow{6}{*}{ HAR-CJ } & 1 & 0.0004 & 0.0005 & 0.0100 & 0.0246 & 0.0220 & 0.0342 & 0.1130 & 0.2036 & 0.1752 & 0.1929 & 0.6856 & 0.8711 \\
\hline & 5 & 0.0002 & 0.0003 & 0.0384 & 0.0657 & 0.0343 & 0.0158 & 0.0523 & 0.1199 & 0.3408 & 0.5656 & 0.5872 & 0.6911 \\
\hline & 10 & 0.0009 & 0.0010 & 0.0004 & 0.0005 & 0.0343 & 0.0458 & 0.0343 & 0.0875 & 0.3408 & 0.5656 & 0.4141 & 0.4200 \\
\hline & 22 & 0.0000 & 0.0000 & 0.0009 & 0.0010 & 0.0343 & 0.0458 & 0.0523 & 0.1199 & 0.0440 & 0.0292 & 0.2767 & 0.4976 \\
\hline & 44 & 0.0000 & 0.0000 & 0.0001 & 0.0001 & 0.0051 & 0.0051 & 0.0343 & 0.0436 & 0.1365 & 0.0936 & 0.1752 & 0.1849 \\
\hline & 66 & 0.0000 & 0.0000 & 0.0022 & 0.0067 & 0.0010 & 0.0015 & 0.0778 & 0.1590 & 0.2217 & 0.1059 & 0.4141 & 0.2262 \\
\hline \multirow{6}{*}{ HAR-PS } & 1 & 0.0009 & 0.0034 & 0.0047 & 0.0121 & 0.0138 & 0.0247 & 0.1602 & 0.2519 & 0.1752 & 0.1929 & 0.4141 & 0.6705 \\
\hline & 5 & 0.0004 & 0.0005 & 0.0384 & 0.0657 & 0.0523 & 0.0593 & 0.0220 & 0.0618 & 0.4141 & 0.6225 & 0.5872 & 0.6911 \\
\hline & 10 & 0.0009 & 0.0010 & 0.0022 & 0.0018 & 0.0778 & 0.0742 & 0.0343 & 0.0875 & 0.2767 & 0.3786 & 0.5872 & 0.4368 \\
\hline & 22 & 0.0000 & 0.0001 & 0.0047 & 0.0129 & 0.0138 & 0.0247 & 0.0343 & 0.0875 & 0.0440 & 0.0292 & 0.2767 & 0.4976 \\
\hline & 44 & 0.0000 & 0.0000 & 0.0001 & 0.0001 & 0.0051 & 0.0051 & 0.0523 & 0.0563 & 0.1050 & 0.0850 & 0.2767 & 0.2143 \\
\hline & 66 & 0.0000 & 0.0000 & 0.0002 & 0.0002 & 0.0010 & 0.0006 & 0.1602 & 0.2519 & 0.2767 & 0.2248 & 0.2767 & 0.2143 \\
\hline & 1 & 0.0002 & 0.0003 & 0.0100 & 0.0246 & 0.0343 & 0.0892 & 0.1602 & 0.2519 & 0.2217 & 0.2106 & 0.4964 & 0.7483 \\
\hline & 5 & 0.0002 & 0.0003 & 0.0384 & 0.0657 & 0.0523 & 0.0593 & 0.0778 & 0.1590 & 0.3408 & 0.5656 & 0.6856 & 0.4297 \\
\hline $\mathrm{R} \Omega \mathrm{C}$ & 10 & 0.0004 & 0.0005 & 0.0004 & 0.0005 & 0.0523 & 0.0593 & 0.0220 & 0.0618 & 0.2217 & 0.3415 & 0.4141 & 0.4200 \\
\hline LHAR-CJ & 22 & 0.0000 & 0.0000 & 0.0022 & 0.0067 & 0.0085 & 0.0173 & 0.0343 & 0.0875 & 0.0796 & 0.0368 & 0.3408 & 0.5588 \\
\hline & 44 & 0.0000 & 0.0000 & 0.0001 & 0.0001 & 0.0085 & 0.0071 & 0.0343 & 0.0436 & 0.1752 & 0.1007 & 0.1752 & 0.1849 \\
\hline & 66 & 0.0000 & 0.0000 & 0.0004 & 0.0015 & 0.0051 & 0.0051 & 0.0778 & 0.1590 & 0.1365 & 0.0936 & 0.3408 & 0.2228 \\
\hline
\end{tabular}

Table C.39: Value-at-Risk backtesting results for $R V$ for Soy Futures. Note that UC is the unconditional coverage test by Kupied ([1995), CC is the conditional coverage test by Christoffersen (I.998), $\alpha$ is the Value-at-Risk level, Position corresponds with the trading position, which is either long (risk of rising prices) or short (risk of falling prices). The test results are presented as $p$-values from the UC and CC test for the forecast horizons 1-, 5-, 10-, 22-, 44-, and 66-days ahead. 


\begin{tabular}{|c|c|c|c|c|c|c|c|c|c|c|c|c|c|}
\hline \multirow{3}{*}{$\begin{array}{l}\alpha \\
\text { Position } \\
\text { Test }\end{array}$} & & \multicolumn{4}{|c|}{0.01} & \multicolumn{4}{|c|}{0.025} & \multicolumn{4}{|c|}{0.05} \\
\hline & & \multicolumn{2}{|c|}{ long } & \multicolumn{2}{|c|}{ short } & \multicolumn{2}{|c|}{ long } & \multicolumn{2}{|c|}{ short } & \multicolumn{2}{|c|}{ long } & \multicolumn{2}{|c|}{ short } \\
\hline & & UC & $\mathrm{CC}$ & $\mathrm{UC}$ & $\mathrm{CC}$ & $\mathrm{UC}$ & $\mathrm{CC}$ & UC & $\mathrm{CC}$ & UC & $\mathrm{CC}$ & UC & $\mathrm{CC}$ \\
\hline \multirow{6}{*}{ RW } & 1 & 0.0001 & 0.0002 & 0.0000 & 0.0000 & 0.0031 & 0.0020 & 0.0002 & 0.0008 & 0.1098 & 0.2260 & 0.0039 & 0.0019 \\
\hline & 5 & 0.1251 & 0.2380 & 0.0000 & 0.0000 & 0.0053 & 0.0168 & 0.0002 & 0.0000 & 0.0173 & 0.0363 & 0.0173 & 0.0091 \\
\hline & 10 & 0.0010 & 0.0026 & 0.0000 & 0.0001 & 0.0018 & 0.0073 & 0.0002 & 0.0004 & 0.0121 & 0.0401 & 0.0026 & 0.0016 \\
\hline & 22 & 0.0103 & 0.0250 & 0.0000 & 0.0000 & 0.0006 & 0.0018 & 0.0000 & 0.0000 & 0.0026 & 0.0097 & 0.0003 & 0.0001 \\
\hline & 44 & 0.0000 & 0.0000 & 0.0000 & 0.0000 & 0.0000 & 0.0000 & 0.0000 & 0.0000 & 0.0007 & 0.0027 & 0.0026 & 0.0016 \\
\hline & 66 & 0.0000 & 0.0000 & 0.0000 & 0.0000 & 0.0000 & 0.0000 & 0.0000 & 0.0000 & 0.0000 & 0.0001 & 0.0000 & 0.0000 \\
\hline \multirow{6}{*}{$\mathrm{AR}$} & 1 & 0.0010 & 0.0026 & 0.0000 & 0.0000 & 0.0018 & 0.0058 & 0.0000 & 0.0000 & 0.0026 & 0.0047 & 0.0001 & 0.0000 \\
\hline & 5 & 0.0206 & 0.0476 & 0.0000 & 0.0000 & 0.0010 & 0.0033 & 0.0000 & 0.0001 & 0.0057 & 0.0113 & 0.0026 & 0.0016 \\
\hline & 10 & 0.0004 & 0.0011 & 0.0001 & 0.0003 & 0.0010 & 0.0043 & 0.0000 & 0.0001 & 0.0057 & 0.0175 & 0.0011 & 0.0009 \\
\hline & 22 & 0.0010 & 0.0035 & 0.0000 & 0.0000 & 0.0002 & 0.0005 & 0.0000 & 0.0000 & 0.0007 & 0.0027 & 0.0001 & 0.0000 \\
\hline & 44 & 0.0000 & 0.0000 & 0.0000 & 0.0000 & 0.0000 & 0.0000 & 0.0000 & 0.0000 & 0.0000 & 0.0000 & 0.0001 & 0.0000 \\
\hline & 66 & 0.0000 & 0.0000 & 0.0000 & 0.0000 & 0.0000 & 0.0000 & 0.0000 & 0.0000 & 0.0000 & 0.0000 & 0.0000 & 0.0000 \\
\hline \multirow{6}{*}{ ARMA } & 1 & 0.0002 & 0.0004 & 0.0000 & 0.0000 & 0.0031 & 0.0020 & 0.0002 & 0.0008 & 0.1098 & 0.2260 & 0.0039 & 0.0019 \\
\hline & 5 & 0.0002 & 0.0004 & 0.0000 & 0.0000 & 0.0053 & 0.0168 & 0.0000 & 0.0000 & 0.0057 & 0.0050 & 0.0017 & 0.0006 \\
\hline & 10 & 0.0103 & 0.0250 & 0.0000 & 0.0000 & 0.0000 & 0.0001 & 0.0000 & 0.0000 & 0.0017 & 0.0065 & 0.0000 & 0.0000 \\
\hline & 22 & 0.0000 & 0.0000 & 0.0000 & 0.0000 & 0.0000 & 0.0000 & 0.0000 & 0.0000 & 0.0001 & 0.0003 & 0.0003 & 0.0002 \\
\hline & 44 & 0.0000 & 0.0000 & 0.0000 & 0.0000 & 0.0000 & 0.0000 & 0.0000 & 0.0000 & 0.0000 & 0.0000 & 0.0002 & 0.0001 \\
\hline & 66 & 0.0000 & 0.0000 & 0.0000 & 0.0000 & 0.0000 & 0.0000 & 0.0000 & 0.0000 & 0.0000 & 0.0000 & 0.0000 & 0.0000 \\
\hline \multirow{6}{*}{ HAR } & 1 & 0.0049 & 0.0123 & 0.0000 & 0.0000 & 0.0018 & 0.0058 & 0.0000 & 0.0001 & 0.0835 & 0.1750 & 0.0039 & 0.0019 \\
\hline & 5 & 0.0206 & 0.0476 & 0.0000 & 0.0000 & 0.0010 & 0.0033 & 0.0000 & 0.0000 & 0.0173 & 0.0178 & 0.0011 & 0.0028 \\
\hline & 10 & 0.0010 & 0.0026 & 0.0000 & 0.0000 & 0.0089 & 0.0274 & 0.0000 & 0.0000 & 0.0039 & 0.0118 & 0.0004 & 0.0003 \\
\hline & 22 & 0.0103 & 0.0250 & 0.0000 & 0.0000 & 0.0001 & 0.0003 & 0.0000 & 0.0000 & 0.0017 & 0.0051 & 0.0000 & 0.0000 \\
\hline & 44 & 0.0000 & 0.0000 & 0.0000 & 0.0000 & 0.0000 & 0.0001 & 0.0000 & 0.0000 & 0.0000 & 0.0001 & 0.0002 & 0.0001 \\
\hline & 66 & 0.0000 & 0.0000 & 0.0000 & 0.0000 & 0.0000 & 0.0000 & 0.0000 & 0.0000 & 0.0000 & 0.0000 & 0.0000 & 0.0000 \\
\hline \multirow{6}{*}{ HAR-J } & 1 & 0.0022 & 0.0058 & 0.0000 & 0.0000 & 0.0031 & 0.0100 & 0.0000 & 0.0001 & 0.0835 & 0.1750 & 0.0057 & 0.0050 \\
\hline & 5 & 0.0206 & 0.0476 & 0.0000 & 0.0000 & 0.0010 & 0.0033 & 0.0000 & 0.0000 & 0.0084 & 0.0078 & 0.0017 & 0.0055 \\
\hline & 10 & 0.0022 & 0.0058 & 0.0000 & 0.0000 & 0.0053 & 0.0168 & 0.0000 & 0.0000 & 0.0084 & 0.0255 & 0.0004 & 0.0001 \\
\hline & 22 & 0.0206 & 0.0431 & 0.0000 & 0.0000 & 0.0001 & 0.0003 & 0.0000 & 0.0000 & 0.0017 & 0.0051 & 0.0000 & 0.0000 \\
\hline & 44 & 0.0000 & 0.0000 & 0.0000 & 0.0000 & 0.0000 & 0.0002 & 0.0000 & 0.0000 & 0.0000 & 0.0002 & 0.0001 & 0.0001 \\
\hline & 66 & 0.0000 & 0.0000 & 0.0000 & 0.0000 & 0.0000 & 0.0000 & 0.0000 & 0.0000 & 0.0000 & 0.0000 & 0.0000 & 0.0000 \\
\hline \multirow{6}{*}{ HAR-CJ } & 1 & 0.0103 & 0.0248 & 0.0000 & 0.0000 & 0.0018 & 0.0011 & 0.0001 & 0.0004 & 0.1098 & 0.1345 & 0.0039 & 0.0019 \\
\hline & 5 & 0.0206 & 0.0476 & 0.0000 & 0.0000 & 0.0018 & 0.0058 & 0.0000 & 0.0002 & 0.0121 & 0.0119 & 0.0017 & 0.0039 \\
\hline & 10 & 0.0010 & 0.0026 & 0.0000 & 0.0000 & 0.0031 & 0.0100 & 0.0000 & 0.0001 & 0.0338 & 0.0955 & 0.0003 & 0.0002 \\
\hline & 22 & 0.0206 & 0.0431 & 0.0000 & 0.0000 & 0.0001 & 0.0003 & 0.0000 & 0.0000 & 0.0017 & 0.0051 & 0.0003 & 0.0002 \\
\hline & 44 & 0.0000 & 0.0000 & 0.0000 & 0.0000 & 0.0000 & 0.0001 & 0.0000 & 0.0000 & 0.0004 & 0.0017 & 0.0001 & 0.0001 \\
\hline & 66 & 0.0000 & 0.0000 & 0.0000 & 0.0000 & 0.0000 & 0.0000 & 0.0000 & 0.0000 & 0.0000 & 0.0000 & 0.0000 & 0.0000 \\
\hline \multirow{6}{*}{ HAR-PS } & 1 & 0.0022 & 0.0058 & 0.0000 & 0.0000 & 0.0006 & 0.0025 & 0.0000 & 0.0002 & 0.0835 & 0.1750 & 0.0121 & 0.0149 \\
\hline & 5 & 0.0394 & 0.0864 & 0.0000 & 0.0000 & 0.0031 & 0.0100 & 0.0000 & 0.0001 & 0.0121 & 0.0119 & 0.0017 & 0.0039 \\
\hline & 10 & 0.0004 & 0.0011 & 0.0000 & 0.0000 & 0.0031 & 0.0100 & 0.0000 & 0.0000 & 0.0084 & 0.0255 & 0.0004 & 0.0003 \\
\hline & 22 & 0.0103 & 0.0250 & 0.0000 & 0.0000 & 0.0000 & 0.0001 & 0.0000 & 0.0000 & 0.0026 & 0.0078 & 0.0001 & 0.0000 \\
\hline & 44 & 0.0000 & 0.0000 & 0.0000 & 0.0000 & 0.0000 & 0.0001 & 0.0000 & 0.0000 & 0.0000 & 0.0001 & 0.0003 & 0.0004 \\
\hline & 66 & 0.0000 & 0.0000 & 0.0000 & 0.0000 & 0.0000 & 0.0000 & 0.0000 & 0.0000 & 0.0000 & 0.0000 & 0.0000 & 0.0000 \\
\hline & 1 & 0.0049 & 0.0123 & 0.0000 & 0.0000 & 0.0010 & 0.0033 & 0.0000 & 001 & 0.0244 & 0.0261 & 0.0057 & 0.0024 \\
\hline & 5 & 0.1251 & 0.2380 & 0.0000 & 0.0000 & 0.0018 & 0.0058 & 0.0000 & 0.0000 & 0.0057 & 0.0050 & 0.0007 & 0.0003 \\
\hline & 10 & 0.0004 & 0.0011 & 0.0000 & 0.0001 & 0.0230 & 0.0672 & 0.0000 & 0.0001 & 0.0057 & 0.0113 & 0.0002 & 0.0001 \\
\hline LHAR-C & 22 & 0.0022 & 0.0071 & 0.0000 & 0.0000 & 0.0006 & 0.0018 & 0.0000 & 0.0000 & 0.0084 & 0.0078 & 0.0001 & 0.0001 \\
\hline & 44 & 0.0000 & 0.0000 & 0.0000 & 0.0000 & 0.0001 & 0.0003 & 0.0000 & 0.0000 & 0.0004 & 0.0017 & 0.0001 & 0.0001 \\
\hline & 66 & 0.0000 & 0.0000 & 0.0000 & 0.0000 & 0.0000 & 0.0000 & 0.0000 & 0.0000 & 0.0000 & 0.0002 & 0.0000 & 0.0000 \\
\hline
\end{tabular}

Table C.40: Value-at-Risk backtesting results for $R V$ for Sugar Futures. Note that UC is the unconditional coverage test by Kupiec ([199.5), CC is the conditional coverage test by Christoffersen ([1998), $\alpha$ is the Value-at-Risk level, Position corresponds with the trading position, which is either long (risk of rising prices) or short (risk of falling prices). The test results are presented as $p$-values from the UC and CC test for the forecast horizons 1-, 5-, 10-, 22-, 44-, and 66-days ahead. 


\begin{tabular}{|c|c|c|c|c|c|c|c|c|c|c|c|c|c|}
\hline \multirow{3}{*}{$\begin{array}{l}\alpha \\
\text { Position } \\
\text { Test }\end{array}$} & & \multicolumn{4}{|c|}{0.01} & \multicolumn{4}{|c|}{0.025} & \multicolumn{4}{|c|}{0.05} \\
\hline & & \multicolumn{2}{|c|}{ long } & \multicolumn{2}{|c|}{ short } & \multicolumn{2}{|c|}{ long } & \multicolumn{2}{|c|}{ short } & \multicolumn{2}{|c|}{ long } & \multicolumn{2}{|c|}{ short } \\
\hline & & UC & $\mathrm{CC}$ & $\mathrm{UC}$ & $\mathrm{CC}$ & $\mathrm{UC}$ & $\mathrm{CC}$ & UC & $\mathrm{CC}$ & UC & $\mathrm{CC}$ & UC & $\mathrm{CC}$ \\
\hline \multirow{6}{*}{ RW } & 1 & 0.0714 & 0.1469 & 0.0022 & 0.0018 & 0.0142 & 0.0430 & 0.0087 & 0.0170 & 0.1404 & 0.1740 & 0.2832 & 0.3725 \\
\hline & 5 & 0.6813 & 0.7924 & 0.0002 & 0.0000 & 0.9375 & 0.4807 & 0.1633 & 0.0255 & 0.9775 & 0.0588 & 0.1082 & 0.0148 \\
\hline & 10 & 0.3234 & 0.5028 & 0.0048 & 0.0004 & 0.3996 & 0.2683 & 0.1154 & 0.0236 & 0.8655 & 0.0646 & 0.0616 & 0.0316 \\
\hline & 22 & 0.1243 & 0.2367 & 0.0022 & 0.0003 & 0.7852 & 0.4398 & 0.0031 & 0.0003 & 0.4223 & 0.4979 & 0.2272 & 0.1003 \\
\hline & 44 & 0.0102 & 0.0246 & 0.0000 & 0.0000 & 0.1633 & 0.1191 & 0.0018 & 0.0000 & 0.2832 & 0.5081 & 0.0616 & 0.0000 \\
\hline & 66 & 0.0391 & 0.0857 & 0.0000 & 0.0000 & 0.0352 & 0.0257 & 0.0005 & 0.0000 & 0.0822 & 0.1862 & 0.0455 & 0.0015 \\
\hline \multirow{6}{*}{$\mathrm{AR}$} & 1 & 0.3234 & 0.5028 & 0.0204 & 0.0412 & 0.1633 & 0.3649 & 0.0536 & 0.0041 & 0.8655 & 0.4850 & 0.5055 & 0.2251 \\
\hline & 5 & 0.6813 & 0.7924 & 0.0022 & 0.0003 & 0.9375 & 0.4807 & 0.2255 & 0.0046 & 0.4573 & 0.0748 & 0.1798 & 0.0046 \\
\hline & 10 & 0.6813 & 0.7924 & 0.0204 & 0.0079 & 0.3996 & 0.2683 & 0.1633 & 0.0255 & 0.6495 & 0.0728 & 0.1404 & 0.0150 \\
\hline & 22 & 0.1243 & 0.2367 & 0.0022 & 0.0003 & 0.6422 & 0.3871 & 0.0052 & 0.0004 & 0.5055 & 0.5770 & 0.1082 & 0.0015 \\
\hline & 44 & 0.0048 & 0.0121 & 0.0000 & 0.0000 & 0.0796 & 0.0588 & 0.0031 & 0.0001 & 0.3481 & 0.4206 & 0.1082 & 0.0004 \\
\hline & 66 & 0.0714 & 0.1469 & 0.0000 & 0.0000 & 0.1633 & 0.1191 & 0.0018 & 0.0000 & 0.5055 & 0.5770 & 0.1082 & 0.0004 \\
\hline \multirow{6}{*}{ ARMA } & 1 & 0.1243 & 0.2367 & 0.0022 & 0.0018 & 0.0142 & 0.0430 & 0.0087 & 0.0068 & 0.1798 & 0.2237 & 0.4223 & 0.4234 \\
\hline & 5 & 0.9122 & 0.8768 & 0.0048 & 0.0004 & 0.3996 & 0.2683 & 0.0796 & 0.0713 & 0.6495 & 0.5031 & 0.2832 & 0.1025 \\
\hline & 10 & 0.3234 & 0.5028 & 0.0001 & 0.0000 & 0.7852 & 0.4398 & 0.0536 & 0.0041 & 0.6961 & 0.7258 & 0.0822 & 0.0050 \\
\hline & 22 & 0.0714 & 0.1469 & 0.0000 & 0.0000 & 0.1633 & 0.3649 & 0.0005 & 0.0000 & 0.2832 & 0.5081 & 0.1404 & 0.0003 \\
\hline & 44 & 0.1243 & 0.2367 & 0.0001 & 0.0000 & 0.5128 & 0.3282 & 0.0087 & 0.0001 & 0.2272 & 0.4442 & 0.0822 & 0.0004 \\
\hline & 66 & 0.0102 & 0.0246 & 0.0000 & 0.0000 & 0.0226 & 0.0664 & 0.0010 & 0.0005 & 0.4223 & 0.4381 & 0.0239 & 0.0001 \\
\hline \multirow{6}{*}{ HAR } & 1 & 0.6813 & 0.7924 & 0.0048 & 0.0132 & 0.3996 & 0.2683 & 0.3039 & 0.0263 & 0.3481 & 0.1708 & 0.3481 & 0.2246 \\
\hline & 5 & 0.4822 & 0.6569 & 0.0004 & 0.0001 & 0.4694 & 0.4528 & 0.1633 & 0.0988 & 0.9775 & 0.0588 & 0.2832 & 0.0039 \\
\hline & 10 & 0.6813 & 0.7924 & 0.0204 & 0.0079 & 0.6422 & 0.3871 & 0.0796 & 0.0210 & 0.8655 & 0.0646 & 0.2272 & 0.0412 \\
\hline & 22 & 0.0714 & 0.1469 & 0.0004 & 0.0001 & 0.7852 & 0.4398 & 0.0226 & 0.0031 & 0.5970 & 0.2971 & 0.1404 & 0.0049 \\
\hline & 44 & 0.0714 & 0.1469 & 0.0000 & 0.0000 & 0.3996 & 0.2683 & 0.0031 & 0.0000 & 0.6961 & 0.8773 & 0.2272 & 0.0003 \\
\hline & 66 & 0.0714 & 0.1469 & 0.0001 & 0.0000 & 0.0796 & 0.0588 & 0.0142 & 0.0001 & 0.5970 & 0.6543 & 0.1404 & 0.0001 \\
\hline \multirow{6}{*}{ HAR-J } & 1 & 0.6813 & 0.7924 & 0.0048 & 0.0132 & 0.3996 & 0.2683 & 0.3039 & 0.0263 & 0.5055 & 0.2523 & 0.3481 & 0.2246 \\
\hline & 5 & 0.4822 & 0.6569 & 0.0004 & 0.0001 & 0.4694 & 0.4528 & 0.2255 & 0.1100 & 0.9775 & 0.0588 & 0.2832 & 0.0039 \\
\hline & 10 & 0.6813 & 0.7924 & 0.0204 & 0.0079 & 0.6422 & 0.3871 & 0.0796 & 0.0210 & 0.8655 & 0.0646 & 0.2272 & 0.0412 \\
\hline & 22 & 0.0714 & 0.1469 & 0.0004 & 0.0001 & 0.7852 & 0.4398 & 0.0226 & 0.0031 & 0.5055 & 0.5770 & 0.1798 & 0.0046 \\
\hline & 44 & 0.0714 & 0.1469 & 0.0000 & 0.0000 & 0.5128 & 0.3282 & 0.0031 & 0.0000 & 0.8011 & 0.7872 & 0.1798 & 0.0003 \\
\hline & 66 & 0.0714 & 0.1469 & 0.0000 & 0.0000 & 0.0796 & 0.0588 & 0.0087 & 0.0001 & 0.8011 & 0.7872 & 0.2272 & 0.0001 \\
\hline \multirow{6}{*}{ HAR-CJ } & 1 & 0.6813 & 0.7924 & 0.0010 & 0.0010 & 0.5128 & 0.3282 & 0.3039 & 0.0263 & 0.5055 & 0.2523 & 0.2832 & 0.1025 \\
\hline & 5 & 0.6813 & 0.7924 & 0.0010 & 0.0002 & 0.3520 & 0.3989 & 0.2255 & 0.1100 & 0.9106 & 0.0524 & 0.2272 & 0.0011 \\
\hline & 10 & 0.3234 & 0.5028 & 0.0391 & 0.0112 & 0.5128 & 0.3282 & 0.0796 & 0.0210 & 0.9775 & 0.0588 & 0.2272 & 0.0412 \\
\hline & 22 & 0.1243 & 0.2367 & 0.0004 & 0.0001 & 0.5128 & 0.3282 & 0.0352 & 0.0036 & 0.2832 & 0.3479 & 0.1798 & 0.0149 \\
\hline & 44 & 0.1243 & 0.2367 & 0.0001 & 0.0000 & 0.3039 & 0.2117 & 0.0142 & 0.0001 & 0.5055 & 0.7538 & 0.0822 & 0.0004 \\
\hline & 66 & 0.1243 & 0.2367 & 0.0001 & 0.0000 & 0.3039 & 0.2117 & 0.0087 & 0.0004 & 0.6961 & 0.0389 & 0.2272 & 0.0011 \\
\hline \multirow{6}{*}{ HAR-PS } & 1 & 0.6813 & 0.7924 & 0.0048 & 0.0132 & 0.6422 & 0.3871 & 0.3039 & 0.0263 & 0.4223 & 0.2098 & 0.2272 & 0.2039 \\
\hline & 5 & 0.4822 & 0.6569 & 0.0004 & 0.0001 & 0.6038 & 0.4907 & 0.2255 & 0.1100 & 0.9775 & 0.0588 & 0.3481 & 0.0034 \\
\hline & 10 & 0.4822 & 0.6569 & 0.0391 & 0.0112 & 0.5128 & 0.3282 & 0.0796 & 0.0210 & 0.8655 & 0.0646 & 0.1082 & 0.0377 \\
\hline & 22 & 0.1243 & 0.2367 & 0.0002 & 0.0000 & 0.6422 & 0.3871 & 0.0226 & 0.0031 & 0.5970 & 0.2971 & 0.2272 & 0.0043 \\
\hline & 44 & 0.0714 & 0.1469 & 0.0000 & 0.0000 & 0.5128 & 0.3282 & 0.0031 & 0.0000 & 0.6961 & 0.8773 & 0.2272 & 0.0003 \\
\hline & 66 & 0.0714 & 0.1469 & 0.0001 & 0.0000 & 0.0796 & 0.0588 & 0.0087 & 0.0000 & 0.6961 & 0.7258 & 0.1404 & 0.0001 \\
\hline & 1 & 0.6813 & 0.7924 & 0.0022 & 0.0018 & 0.6422 & 871 & 039 & 263 & 0.6961 & 0.3425 & 0.6961 & 0.2045 \\
\hline & 5 & 0.9122 & 0.8768 & 0.0022 & 0.0003 & 0.2539 & 0.3348 & 0.2255 & 0.1100 & 0.9775 & 0.0588 & 0.4223 & 0.0112 \\
\hline & 10 & 0.4822 & 0.6569 & 0.0391 & 0.0112 & 0.9059 & 0.5048 & 0.2255 & 0.0265 & 0.7554 & 0.0693 & 0.2272 & 0.0144 \\
\hline LHAR-C & 22 & 0.1243 & 0.2367 & 0.0010 & 0.0002 & 0.9375 & 0.4807 & 0.0796 & 0.0210 & 0.6961 & 0.3425 & 0.2832 & 0.0404 \\
\hline & 44 & 0.2058 & 0.3572 & 0.0002 & 0.0000 & 0.5128 & 0.3282 & 0.0352 & 0.0001 & 0.8011 & 0.7872 & 0.2832 & 0.0010 \\
\hline & 66 & 0.3234 & 0.5028 & 0.0004 & 0.0001 & 0.9375 & 0.4807 & 0.0142 & 0.0005 & 0.8655 & 0.0646 & 0.2832 & 0.0010 \\
\hline
\end{tabular}

Table C.41: Value-at-Risk backtesting results for $R V$ for Wheat Futures. Note that UC is the unconditional coverage test by Kupiec (19.9.5), CC is the conditional coverage test by Christoffersen ([1998), $\alpha$ is the Value-at-Risk level, Position corresponds with the trading position, which is either long (risk of rising prices) or short (risk of falling prices). The test results are presented as $p$-values from the UC and CC test for the forecast horizons 1-, 5-, 10-, 22-, 44-, and 66-days ahead. 